
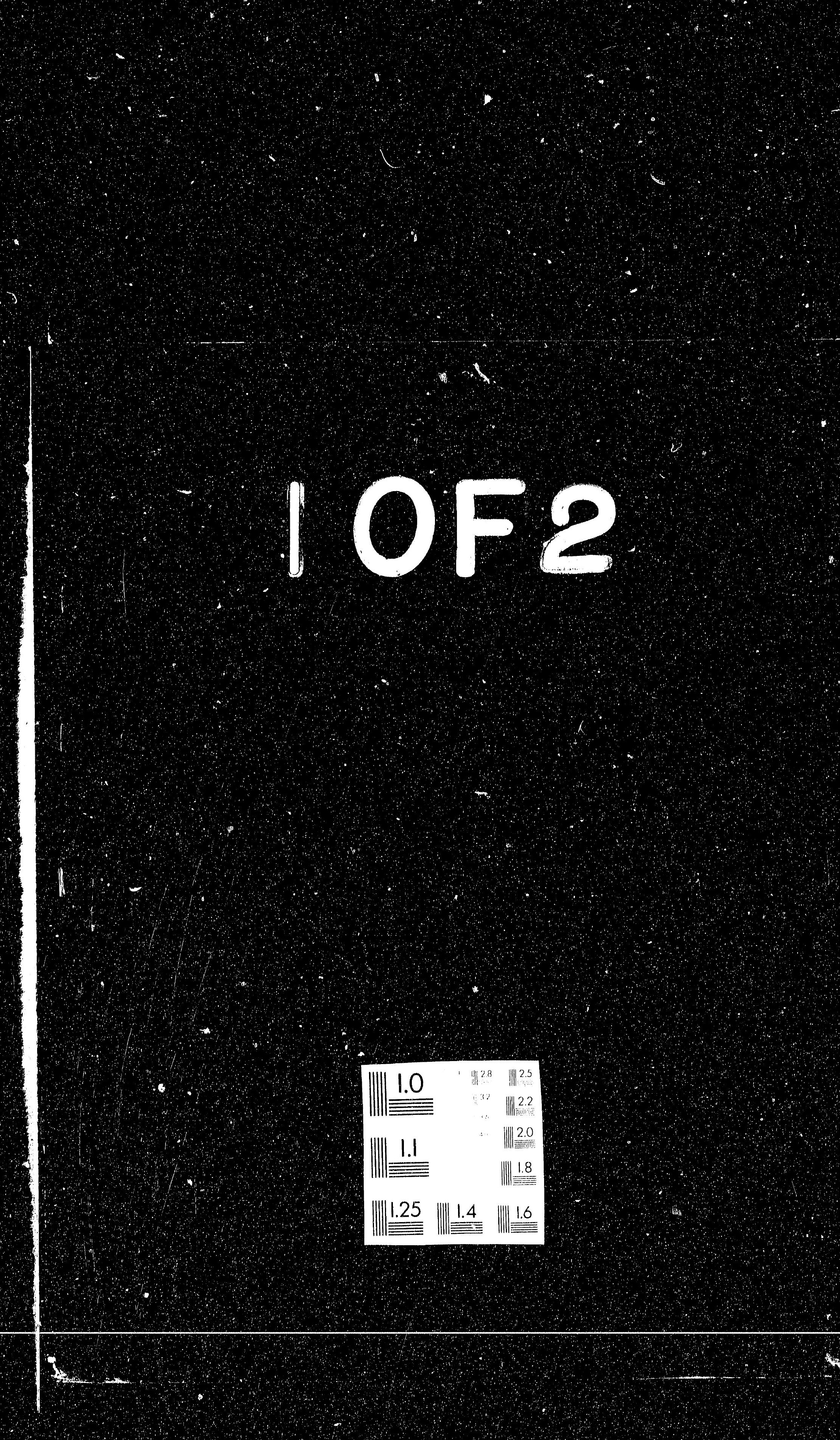

Figure 6.5 Two-loop rainbow self-energy contribution to $\sigma \rightarrow f \bar{f}$. $\delta m, \delta^{(2)} m$ denote the one- and two-loop self-energy mass correction, respectively. I corresponds to a countert- 


\title{
PERTURBATION THEORY IN LIGHT-CONE QUANTIZATION
}

\author{
ALEX LANGNAU
}

SLAC-Report-385

JANUARY 1992

Prepared for the Department of Energy under contract number DE-AC03-76SF00515

Stanford Linear Accelerator Center Stanford University • Stanford, CA 94309 
This document and the material and data contained therein, was developed under sponsorship of the United States Government. Neither the United States nor the Department of Energy, nor the Leland Stanford Junior University, nor their employees, nor their respective contractors, subcontractors, or their employees, makes any warranty, express or implied, or assumes any liability or responsibility for accuracy, completeness or usefulness of any information, apparatus, product or process disclosed, or represents that its use will not infringe privately-owned rights. Mention of any product, its manufacturer, or suppliers shall not, nor is it intended to, imply approval, disapproval, or fitness for any particular use. A royalty-free, nonexclusive right to use and disseminate same for any purpose whatsoever, is expressly reserved to the United States and the University. 


\title{
PERTURBATION THEORY IN LIGHT-CONE QUANTIZATION*
}

\author{
Alex Langnau \\ Stanford Linear Accelerator Center \\ Stanford University \\ Stanford, CA 94309
}

January 1992

Prepared for the Department of Energy under contract number DE-AC03-76SF00515.

Printed in the United States of America. Available from the National Technical Information Service, U.S. Department of Commerce, 5285 Port Royal Road, Springfield, VA 22161.

* Ph.D thesis. 
To my parents and Susan 
It's never what you think; it's what you do.

Diane Reed, Bruce King

(Hoover Institution) 


\section{Acknowledgements}

I would like to express my gratitude to my advisor Prof. Brodsky, for his tireless dedication to this work, and even more for his unparalleled combination of enthusiasm and patience. I wish to acknolwledge Matthias Burkardt, without his constant support and devotion, this work would have never been possible. It is a pleasure to recognize Nina Adelman, Hela Kohrs, Prof. Blankenbecler, Michael Göring, Andreas Müller and Prof. Nachtmann who made this possible. Then there is Hoover. There is a special place in my heart for all the members of the Hoover institution. These friends were able to inject some sanity into what would otherwise be an insane situation. We are all Hoover, and Hoover will always be part of us. 


\section{Table of Contents}

Chapter

Page

Acknowledgements $\ldots \ldots \ldots \ldots \ldots \ldots \ldots \ldots \ldots \ldots \ldots \ldots \ldots, \mathrm{v}$

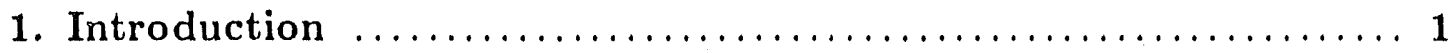

2. Light-Cone Perturbation Rules $\ldots \ldots \ldots \ldots \ldots \ldots \ldots \ldots \ldots \ldots$

3. Perturbation in Light-cone Quantization $\ldots \ldots \ldots \ldots \ldots \ldots \ldots$

3.1. A general algorithm for generating LCPTh $\ldots \ldots \ldots \ldots \ldots \ldots$

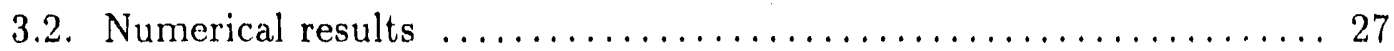

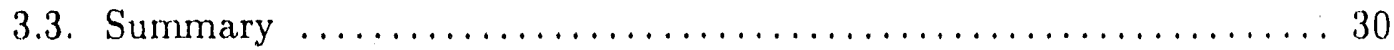

4. Ultraviolet Regularization of Light-Cone Hamilton Perturbation Theory: Application to the Anomalous Magnetic Moment of the Electron in Light-cone Gauge $\ldots \ldots \ldots \ldots \ldots \ldots \ldots \ldots \ldots$

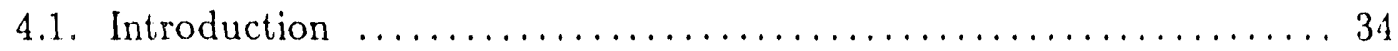

4.2. Light-Cone quantization in Feynman gauge $\ldots \ldots \ldots \ldots \ldots \ldots$

4.3. Light-Cone quantization in light-cone gauge $\ldots \ldots \ldots \ldots \ldots \ldots \ldots 4$

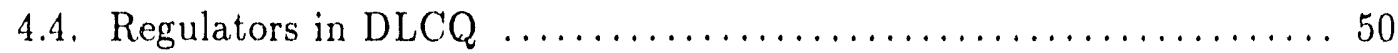

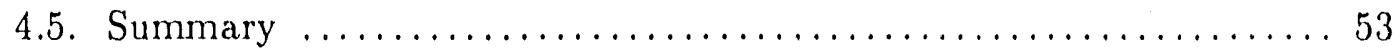

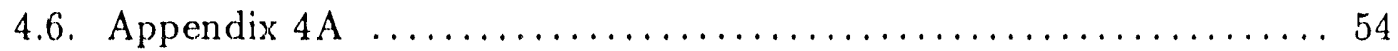

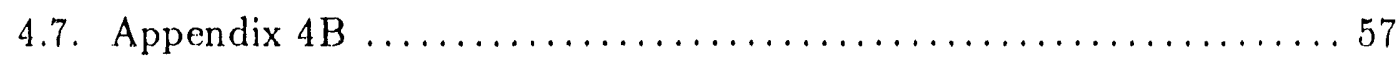

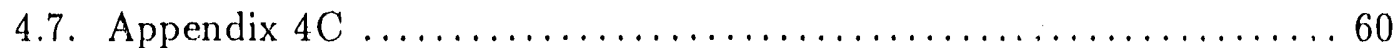

5. A Hamilton Formulation of QED $(2+1)$ on the Light-Cone ....67

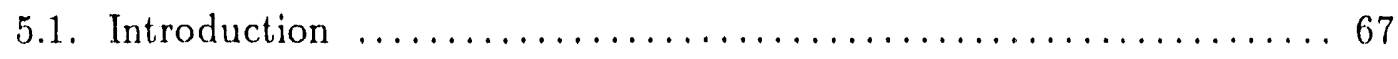

5.2. Pauli-Villars regularization of the light-cone

quantized Yukawa model $\ldots \ldots \ldots \ldots \ldots \ldots \ldots \ldots \ldots \ldots, \ldots, \ldots$ 
5.3. Hamiltonian formulation for $Q E D_{2+1}$ in the light-cone gauge (Pauli-Villars regularization) $\ldots \ldots \ldots \ldots \ldots \ldots \ldots \ldots \ldots \ldots \ldots \ldots \ldots$

5.4. Renormalization using noncovariant counterterms ........... 77

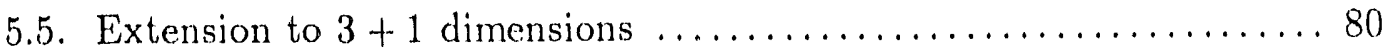

5.6. Summary and Conclusion $\ldots \ldots \ldots \ldots \ldots \ldots \ldots \ldots \ldots \ldots \ldots$

5.7. Appendix 5A: The Pauli-Villars regularized Hamiltonian for $\mathrm{QED}_{2+1} \ldots \ldots \ldots \ldots \ldots \ldots \ldots \ldots \ldots \ldots \ldots \ldots \ldots \ldots$

5.8. Appendix 5B: The two-loop self energy in Yukawa $D_{\perp}+2 \ldots \ldots \ldots 87$

6. Rotational Invariance in Light-Cone Quantization .......... 92

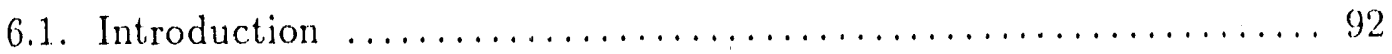

6.2. Breakdown of covariance at the one-loop level and addition of noncovariant counterterms $\ldots \ldots \ldots \ldots \ldots \ldots$

6.3. Breakdown of covariance at the two-loop level ............... 99

6.4. Surface and zero mode contributions $\ldots \ldots \ldots \ldots \ldots \ldots \ldots \ldots \ldots$

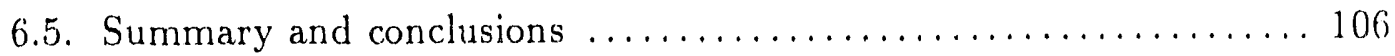

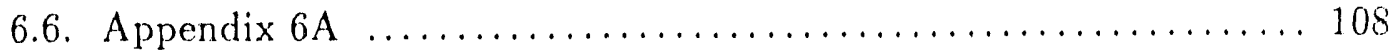

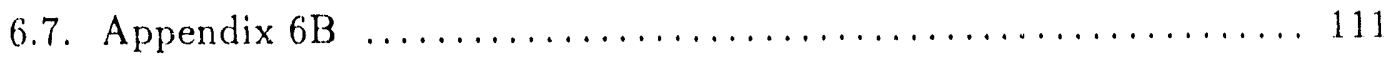

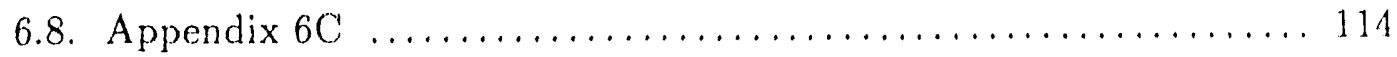

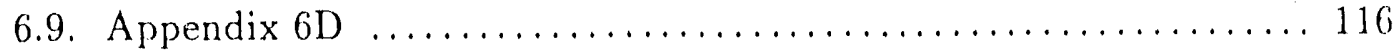

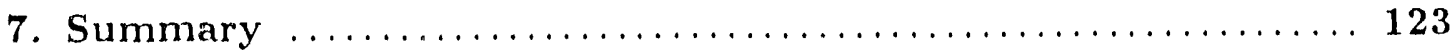




\section{Table of Tables}

Table

Page

Table 3.1 Result $(\delta a)$ of the numerical integration for diagram $1+2$ after mass renormalization in units of $(\alpha / \pi)^{2}$. The data converge for large values of the Pauli-Villars cut-off $\Lambda$ (in units of the electron mass).

Table 3.2 Numerical results $(\delta a)$ for the two-loop diagrams of Fig. 3.7. The results are compared with the analytic answer by Petermann.

Table 3.3 Numerical results $(\delta a)$ for the sixth-order contributions of the diagrams in Fig. 3.8. The results are compared with the results given by Brodsky and Kinoshita.

Table 4.1 Total answer for the electon anomaly to fourth order in light-cone gauge for different values of the gauge regulator. The analytic Feynman answer is given by $a=-131.4 \ldots(\alpha / \pi)^{2}$.

Table 6.1 Self-energy contribution to $\sigma \rightarrow f \bar{f}$ in two loops. $a_{2}$ describes the contribution from the instantaneous diagrams (Fig. 6.7), which violate rotational invariance. $a_{1}$ is the result of the numerical integration of the residual self-energy diagrams. 
Table

Table 6.2 Result of the numerical integration of the ladder vertex correction to $\sigma \rightarrow f \bar{f}$ (Fig. 6.6). A rotational invariant answer is obtained for both sets. 101

Table 6.3 Total one-loop contribution to $\sigma \rightarrow f \bar{f}$. 110 


\section{Table of Figures}

Figure

Page

Figure 2.1 One-loop contribution to the decay $t \rightarrow b W$.

Figure 3.1 Two-loop QED cross diagram. The momenta $p_{i}$ and $k_{j}$ correspond to the internal momenta of the $\mathrm{i}$-th fermion and $\mathrm{j}$-th photon, respectively.

Figure 3.2 Light-cone time-ordering contributing to the cross diagram.

Figure 3.3 Flow chart for the automatic computation of QED amplitudes.

Figure 3.4 Two-loop "corner" diagram.

Figure 3.5 Example for a vacuum fluctuation to the cross diagram. 24

Figure 3.6 Example for a set of light-cone time-orderings which correspond to a sixth-order Feynman diagram.

Figure 3.7 Six Feynman diagrams and the corresponding light-cone time-orderings contributing to the fourth-order anomalous magnetic moment to the electron.

Figure 3.8 Sixth-order Feynman diagrams containing one-loop vacuum polarization. 
Figure

Page

Figure 4.1 Feynman diagrams $F$ with corresponding light-cone timeordered diagrams contributing to the electron anomalous magnetic moment $a=(g-2) / 2$ to fourth order. 36

Figure 4.2 n-photon jellyfish graph.

Figure 4.3 Powercounting for the $n=2$ jellyfish diagram leads to a. logarithmic divergence.

Figure 4.4 One-loop correction to Compton scattering.

Figure 4.5 Two-loop rainbow गlf-energy diagram.

Figure 4.6 The two-loop self-energy contribution of the electron is expected to be of the form $A+B p$, where $p$ corresponds to the external fermion momentum. The result res shows the extraction of $B$ by means of the various components of $p$.

Figure 4.7 Contributions res of single LCP'Th diagrams to the anomalous magnetic moment of the electron $a=(g-2) / 2$ to fourth order in light-cone gauge for different values of the light-cone gauge cutoff $\epsilon$ [24].

Figure 4.8 Fourth-order correction to the electron anomaly in lightcone gauge for a different value of its gange regulator. The analytic Feynman answer is given by -137.2 for $\alpha / 2 \pi=10$. 48 
Figure

Page

Figure 4.9 Instantaneous contribution to the external wavefunction correction in light-cone gauge.

Figure 4.10 Correction to $e^{+} e^{-}$scattering. 52

Figure 4.11 Mass correction to electron Compton scattering. 54

Figure 4.12 Double ins'antaneous diagram to electon Compton scattering.

Figure 4.13 Ladder diagram contribution to the electron anomaly in fourth order.

Figure 5.1 Rainbow diagram contribution to the two-loop fermion self-energy in the Yukawa model.

Figure 6.1 Tree level matrix element for the decay $\sigma \rightarrow f \bar{f}$. The dashed line represents a heavy boson with mass $\lambda$ at rest: $p^{+}=p^{-}, p_{\perp}=0$. The sum runs over the fermion (mass $m$ ) spin labels $s_{f}, s_{\bar{f}}$.

Figure 6.2 Fourth-order contributions to $\sigma \rightarrow f \bar{f}$. The $\delta m$ insertion represents the one-loop mass counterterm.

Figure 6.3 Typical contribution to the vertex correction of $\sigma \rightarrow f \bar{f}$. 95

Figure 6.4 Instantaneous contributions to the external self-energy. 97 
Figure

Page

Figure 6.5 Two-loop rainbow self-energy contribution to $\sigma \rightarrow f \bar{f}$. $\delta m, \delta^{(2)} m$ denote the one- and two-loop self-energy mass correction, respectively. I corresponds to a counterterm which restores rotational invariance at the one-loop level.

Figure 6.6 Two-loop ladder vertex correction to $\sigma \rightarrow f \bar{f}$. Six timeorderings add up to the covariant answer.

Figure 6.7 Instantaneous self-energy correction in two loops. Momentum labels are assigned as indicated.

Figure 6.8 $n+1$ loop rainbow self-energy correction.

Figure 6.9 Self-energy diagram in one loop. 108

Figure 6.10 One-loop mass correction to the self-energy. 


\section{Introduction}

Perhaps the most outstanding problem in quantum field theory is to compute the bound state spectrum and relativistic wavefunctions of hadrons at strong coupling. In quantum chromodynamics (QCD) one needs a practical computational method which not only determines the hadronic spectra, but also provides nonperturbative hadronic matrix elements.

Lattice gauge theory, in which the Feynman path integral is evaluated on a discrete spacetime grid, provides an appropriate tool for such calculations. For strong coupling, it leads to an appealing description of confinement. Numerical results in general have, at least qualitatively, been consistent with experiment, and there is little doubt their accuracy will improve with increasing computing power.

Nevertheless, it is necessary to develop other methods which are perhaps more intuitive and less time consuming than the lattice gauge theory approach. In addition, it is particularly important to compute the relativistic wavefunctions noeded to calculate structure functions, form factors and other hadronic matrix elements. $A$ step in this direction has been undertaken by a method known as Discrete Light-Conc Quantization (DLCQ). So far, the theory has been applied mainly to the elucidation of quantum field theories in one space and one time dimension. In $1+1$ dimensional QCD, for example, the full spectra and wavefunctions could be obtained, using the DLCQ method [1]. These results, which required only a minimal numerical effort, are in agreement with other calculations when available. The success of DLCQ, as well as a similar approach, known as Light-front Tamm-Dancoff method [2], provide the hope for solving field theories in $3+1$ dimensions.

However, the transition to dimensions higher than $1+1$ is anything but straightforward. Some of the reasons are the following: 
- Theories in $1+1$ dimensions, quantized on the light-cone, are manifestly covariant. This is because the operator of boost transformations, which is a kinematic Poincare operator in light-cone quantization, is the only generator of continuous Lorentz transformations. This is generally not the case in higher dimensional field theories, since the underlying Poincare group includes certain rotation operators, which are dynamical in the light-cone formulation. 'Thus, the recovery of Lorentz invariant physical observables is a nontrivial problem in light-cone quantized theories beyond $1+1$ dimensions (as for any form of Hamilton dynamics) [3] .

- The Hamiltonian formulation of gauge theories in $1+1$ dimensions is effectively gauge invariant [4]. However, in higher dimensions the regularization imposed in such a formalism will generally spoil gauge invariance, since the gauge field quanta become a dynamical degree of freedom of the theory. Unless a careful regularization is imposed, gauge invariant amplitudes are not recovered in the continuum limit.

- Simple theories like the Yukawa model or gauge theories in $1+1$ dimensions are superrenormalizable. In $3+1$ dimensions, however, a renormalization scheme to all orders in the coupling constant and masses must be imposed for these theories in order to ensure a consistent treatment of their short distance behavior.

- The number of degrees of freedom in $3+1$ dimensional theories is drastically enhanced compared to the $1+1$ dimensional world.

Thus, a thorough investigation of light-cone properties which are characteristic for higher dimensions is very important. The easiest way of addressing these issues is by analysing the perturbative structure of light-cone field theories first. Perturbative studies cannot be substituted for an analysis of problems related to a nonperturbative approach. However, in order to lay down groundwork for upcoming 
nonperturbative studies, it is indispensable to validate the renomalization methods at the perturbative level, i.e., to gain control over the perturbative treatment first. A clear understanding of divergences in perturbation theory', as well as their mumerical treaiment, is a necessary first step towards formulating such a program.

The first objective of this dissertation is to clarify this issue, at least in second and fourth-order in perturbation theory. The work in this dissertation can provide guidance for the choice of counterterms in DLCQ or the Tamm-Dancoff approach.

A second objective of this work is the study of light-cone perturbation theory (LCPTh) as a competitive tool for conducting perturbative Feynman diagram calculations. Feynman perturbation theory has become the most practical tool for computing cross sections in high energy physics and other physical properties of field theory. Although this standard covariant method has been applied to a great range of problems, computations beyond one-loop corrections are very difficult. A number of examples of two-loop and higher calculations using Feymman methods are given in Ref. [5].

Because of the algebraic complexity of the Feynman calculations in higher-order perturbation theory, it is desirable to automatize Feynman diagram calculations so that algebraic manipulation programs can carry out almost the entire calculation. This thesis presents a step in this direction. The technique we are elaborating on here is known as light-cone perturbation theory (LCPTh) [6-8].

LCPTh is similar to ordinary time-ordered perturbation theory, familiar in both nonrelativistic quantum mechanics and quantum field theory, where each time-ordered amplitude is constructed from a product of energy denominators and interaction vertices. The covariant Feynman amplitude is, in principle, obtained from the sum of time-ordered noncovariant graphs with the same topology. Instead of ordinary tinke, the LCPTh evolution parameter is the time along the light-cone $\tau=t-z / c$. Thr $\tau$ - ordered amplitudes are each invariant under a large class of Lorentz boosts, so 
that each $\tau$-ordered amplitude is itself frame-independent with respect to those symmetries.

A straightforward way of relating the LCPTh amplitudes to the Feyman rules is by changing variables of the independent loop momenta $k$ in a Feynman integral according to $[9,10]$

$$
\int d^{4} k \rightarrow \frac{1}{2} \int d k^{+} d^{2} k_{\perp} d k^{-}
$$

with $k^{ \pm}=k^{0} \pm k^{3}$, and performing the integration over $k^{-}$. The residues give the LCP'Th amplitudes. Alternatively, these amplitudes can be obtained directly from the Hamiltonian formalism derived at fixed $\tau$. Thus by constructing LCPTh directly, only a three dimensional integral has to be performed for each loop. Since the complex contour integrations over energy or $k^{-}$do not occur, the formalism is immediately suitable for numerical treatment.

The price to pay for the simple features of LCPTh is that every Feynman diagram with $n$ vertices gets decomposed into a set of light-cone time-ordered diagrams. However, unlike time-ordered perturbation theory (which can be obtained after performing the $k^{0}$ integration of the independent loop momenta), the number of light. cone tirne-orderings corresponding to the Feynman amplitude is considerably smaller than $n$ ! For example, in the case of the fourth-order $(\alpha / \pi)^{2}$ correction to the electron's anomalous moment ( without vacuum polarization), there are 516 individual time-ordered contributions, but only 8 of them are nonvanishing in the light-cone formalism. This example will be discussed further in the following sections.

There are a number of other advantages of the light-cone perturbation theory formalism.

- Since each amplitude describes the propagation of on-mass-shell particles with a specific time-ordering, the physical meaning of each LCP'Th amplitude is 
immediate. General properties such as unitarity and cluster decomposition theorems become explicit.

- If one quantizes in a physical gauge, all intermediate states correspond to the propagation of physical particles with positive metric. The physical variables used to describe jets or particles in high energy physics have an immediate interpretation in terms of the LC variables.

- The cancellation of infrared divergences is immediate and can be carried out for contributions with the same LC time-ordering.

- The LC quantization of quantum chromodynamics leads to a direct physical interpretation of the theory. The implementation of current algebra becomes essentially a kinematic problem [11-13]. The current matrix elements $J^{+}$ needed to compute form factors and structure functions can be written as diagonal matrix elements of the light-cone wavefunctions, since such currents do not couple to vacuum fluctuations in the LC quantized theory $[13,14]$.

This thesis is organized as follows :

- Chapter 1 gives an introduction.

- Chapter 2 lists light-cone perturbation theory rules.

- Chapter 3 presents a new algorithm for the automatic computation of Feynman diagram amplitudes. Once the topology of a diagram is defined, the algorithm constructs all corresponding light-cone time-orderings. We explore the method for two- and three-loop calculations in QED. This chapter lays down the computational techniques used in the thesis.

- Chapter 4 discusses an ultraviolet regularization and renormalization procedure of light-cone perturbation theory, which is suitable for numerical application. In this sense, Chapter 4 augments the discussion of Chapter 3. 'The fourth-order' correction to the anomalous magnetic moment of the clectron is computed in 
light-cone gauge. Several regularizations of the associated gauge singularity are explored. Local counterterms are constructed to remove the quadratic lightcone divergences from the formalism. Problems of the Discrete Light.-Cone Quantization (DLCQ) and the light-front Tamm-Dancofr approach, beyond the one photon exchange, are also described.

- Chapter 5 elaborates upon the problems of Chapter 4 in the context of nonperturbative methods, such as DLCQ. The light-cone Hamiltonian for $Q E D_{2+1}$ consistent with covariant and gauge-invariant perturbation theory is constructed. Extension to gauge theories in $3+1$ physical dimensions is also described.

- Chapter 6 investigates specific features of a perturbation expansion in lightcone field theory. The decay of a heavy scalar particle at rest, in the Yukawa model, at the one- and two-loop level, is studied. It is shown explicitly that naive light-cone quantization leads to a violation of rotational invariance. Noncovariant counterterms are constructed in detail to restore Lorentz covariance. An analysis of surface and zero mode contributions clarifies the origin of the problem.

- Chapter 7 summarizes this work and outlines possible future work in this field. It may be used as a expanded abstract of the thesis.

This thesis is structured such that each chapter can be read mostly independently' from other chapters. The advantage is that the reader, whose interest in this work is focused on LCPTh as a competitive tool for standard Feyman diagram calculations, needs to concentrate mainly on Chapters 2, 3 and 4. Those interested in the consequences of this work for applications in DLCQ should focus on Chapter 5 (and the last subsection of Chapter 4). For those interested in the results of this thesis in general the focus during the first reading should be on Chapters 3 and 6 .

Chapters 3, 4, 5 and 6 of this thesis have been either published, accepted or submitted for publication [15]. 


\section{REFERENCES for CHAPTER 1}

[1] Phys. Rev. D35, 1493 (1987); K. Hombostel, Ph.I), thesis, SLAC-REPCR' 0333 (1989); M. Burkardt, Nucl. Phys. A504, 762 (1.989).

[2] A. Harindranath and R. J. Perry, Phys. Rev. D43, 492 (1991); R. J. Perry and A. Harindranath, Phys. Rev. D43, 4051 (1991).

[3] We note that even in $1+1$ dimensional theories, when quantized on the light-cone, do not manifestly preserve parity. In fart, the fermion one-loop selfenergy in the Yukawa model in $1+1$ dimensions is logarithmically divergent, unless a careful regularization is imposed. This is duc to the breakdown of parity and in contrast to a covariant theory where the fermion self-energy is finite.

[4] By effective gauge invariance we mean that gange invariance can be recoverel in the continuum limit if a reasonable cut-off is introduced.

[5] T. Kinoshita, Quantum Electrodynamics. N.J, World Scientific, 1990 ; 'T. Ki. noshita, B. Nizic, Y. Okannoto, Phy's. Rev. D41, 593 (1990); S. C. Ciorishmy, A. L. Kataev, and S. A. Larin, Phys. Lett. B212, 238 (1988).

[6] David E. Soper, SLAC-PUB-0137 (1971).

[7] John B. Kogut, Davison E. Soper, Phys. Rev. D1, 2901 (1970).

[8] G. P. Lepage and S. J. Brodsky, Phys. Rev. D22, 2157 (1980).

[9] S. J. Brodsky, R. Roskies and R. Suaya, Phys. Rev. D8, 4574 (1973).

[10] M. G. Schmidt, Phys. Rev. D9, 408 (1974).

[11] Y. Frishman, Phys. Reports 13C, 1 (1974).

[12] J. D. Bjorken, J. B. Kogut and D. E. Soper, Phys. Rev. D3, 1382 (1971).

[13] S. D. Drell, D. J. Levy, and 'T. M. Yan, Phys. Rev. Letl, 22, 7.4 (196(i)); Phỵs. Rev. 187, 2159 (1969). 
[14] H. C. Pauli and S. J. Brodsky, Phys. Rev. D32, 1993 (1987); 2001 (1987); T. Eller, H.-C. Pauli and S. J. Brodsky, Phys. Rev. D35, 1493 (1987); K. Hornbostel, Ph.D. thesis, SLAC-REPORT-0333 (1989); M. Burkardt, Nucl. Phys. A504, 762 (1989); R. J. Perry, A. Harindranath and K. G. Wilson, Phys. Rev. Lett. 65, 2959 (1990); R. J. Perry, A. Harindranath, Phys. Rev. D43, 492 (1991); R. J. Perry, A. Harindranath, Phys. Rev. D43, 4051 (1991).

[15] Chapter 5 has been published: M. Burkardt and A. Langnau, Phys. Rev. D44, 1187 (1991). Chapter 6 has been accepted for publication in the same journal (M. Burkardt and A. Langnau, SLAC-PUB-5611). Chapter 3 has been submitted to the Journal of Computational Physics (A. Langnau and S. J. Brodsky, SLAC-PUB-5667). Chapter 4 has been submitted for publication in Phys. Rev. D (A. Langnau and M. Burkardt, SLAC-PUB-5668). 


\section{Light-Cone Perturbation Theory Rules}

In this section we present light-cone perturbation theory rules which are adopted from Ref. [1]. The light-cone Green's functions are the probability amplitudes that a state starting in Fock state $|i\rangle$ ends up in Fock state $|f\rangle$ a (light-cone) time $\tau$ later [3]

$$
\begin{aligned}
\langle f \mid i\rangle G(f, i ; \tau) & \equiv\left\langle f\left|e^{-i H_{L C T / 2}}\right| i\right\rangle \\
& =i \int \frac{d \epsilon}{2 \pi} e^{-i \epsilon \tau / 2} G(f, i ; \epsilon)\langle f \mid i\rangle
\end{aligned}
$$

where Fourier transform $G(f, i ; c)$ can be written

$$
\begin{aligned}
\langle f \mid i\rangle G(f, i ; \epsilon)= & \left\langle f\left|\frac{1}{\epsilon-H_{L C}+i 0_{+}}\right| i\right\rangle \\
= & \langle f| \frac{1}{\epsilon-H_{0}+i 0_{+}}+\frac{1}{\epsilon-H_{0}+i 0_{+}} V \frac{1}{\epsilon-H_{0}+i 0_{+}} \\
& +\frac{1}{\epsilon-H_{0}+i 0_{+}} V \frac{1}{\epsilon-H_{0}+i 0_{+}} V \frac{1}{\epsilon-H H_{0}+i 0_{+}}+\ldots|i\rangle .
\end{aligned}
$$

$H_{L C}$ and $H_{0}$ denote the full and free Hamiltonian respectively. The rules for $\tau$ ordered perturbation theory follow immediately when $\left(e-H_{0}\right)^{-1}$ is replaced by its spectral decomposition

$$
\frac{1}{\epsilon-H_{0}+i 0_{+}}=\sum_{n, \lambda_{1}} \int \tilde{\prod} \frac{d k_{i}^{+} d^{2} k_{\perp i}}{16 \pi^{3} k_{i}^{+}} \frac{\left|n: \underline{k}_{i}, \lambda_{i}\right\rangle\left\langle n: \underline{k}_{i}, \lambda_{i}\right|}{\epsilon-\sum_{i}\left(k_{\perp}^{2}+m^{2}\right)_{i} / k_{i}^{+}+i 0_{+}} .
$$

The sum becomes a sum over all states $n$ intermediate between two interactions.

To calculate $G(f, i ; \epsilon)$ perturbatively then, all $\tau$-ordered diagrams must be considered, the contribution from each graph computed according to the following rules:

1. Assign a momentum $k^{\mu}$ to each line such that the total $k^{+}, k+$ are conserverl at each vertex, and such that $k=m^{2}$, i.e., $k^{-}=\left(k_{1}^{2}+m^{2}\right) / k^{+}$.

2. Include a factor $\Theta\left(k^{+}\right)$for each line. 
3. For ench gluon (photon) line include a factor $d_{\mu \nu}^{(k)} / k^{+}$where $d_{\mu \nu}$ is the (gauge dependent) polarization sum. In Feymman gauge $d_{\mu \nu}$ equals $-g_{\mu \nu}$. In lightcone gauge $\eta \cdot A=A^{+}=0$,

$$
\begin{aligned}
d_{\mu \nu}^{(k)} & =\sum_{\lambda=1,2} \epsilon_{\mu}^{*}(k, \lambda) \epsilon_{\nu}(k, \lambda) \\
& =-g_{\mu \nu}+\frac{\eta_{\mu} k_{\nu}+\eta_{\nu} k_{\mu}}{\eta \cdot k},
\end{aligned}
$$

where $k \cdot \epsilon=\eta \cdot k=0$. For a regularization of the gauge singularity at $\eta \cdot k=0$ (see Refs. [4-6]).

4. The gluon (photon)-fermion vertex is

$$
\epsilon_{0} \gamma^{\mu}
$$

The trigluon vertex is

$$
-e_{0}\left[(p-q)^{\rho} g^{\mu \nu}+(q-k)^{\prime \prime} g^{\rho \nu}+(k-p)^{\prime \prime} g^{\mu \prime \rho}\right]
$$

and the four-gluon vertex is

$$
\epsilon_{0}^{2}\left(g^{\mu \rho} g^{\nu \sigma}-g^{\mu \sigma} g^{\nu \rho}\right)
$$

Generally there are three independent ways of inserting the four-gluon vertex; all must be included.

5. For each intermediate state there is a factor

$$
\frac{1}{\sum_{i n c} k^{-}-\sum_{i n t e r m} k^{-}+i c}
$$

where the sums in the "energy denominators" are over the light-cone "cnergics," $k^{-}$, of the incident (inc) and intermediate (interm) particles. 
6. In Feynman gauge, ghosts loops occur. For each ghost line include a factor $-\left[\Theta\left(k^{+}\right)\right] / k^{+}$. The gluon-ghost vertex is $e_{0} k^{\nu}$.

7. The fermion propagator consists of two parts:

- A propagating piece

$$
\frac{1}{k^{+}}(k+m), \quad \frac{1}{k^{+}}(-k+m)
$$

where the first and second term correspond the propagation of a fermion and aritifermion, respectively.

- An instantaneous contribution

$$
\frac{\gamma^{+}}{2 k^{+}}
$$

Also the gluon (photon) propagator in light-cone gange has an instant aneons part $\left(\eta^{\mu} \eta^{\nu}\right) / k^{+2}$, as does the ghost propagator; In each case, the instantaneous propagat,or can be absorbed into the regular propagator by replacing $k$, the momentum associated with the line, by

$$
\tilde{k}=\left(k^{+}, \sum_{\text {inc }} k^{-}-\sum_{\text {interm }}^{\prime} k^{-}, k\right)
$$

in the numerator of those diagrams in which the fermion, gluon (photon), or ghost propagates only over a single time interval. Here $\sum_{i n c}$ denotes summation over all initial particles in the diagram, while $\sum_{\text {interm }}^{\prime}$ denotes summation over all particles in the intermediate state other than the particle of interest. Thus, in light-cone gauge, $\hat{k}$ replaces $k$ in the polarization sum $d_{\mu \nu}^{(k)}$, as well as in the trigluon coupling, for gluons appearing in a single intermediate statc. Similarly, $\pm f+m$ is replaced by $\pm \tilde{k}+m[7]$. 
8. Integrate $\int d k^{+} d^{2} k_{\perp} / 16 \pi^{3}$ over each independent $k$.

9. Include a factor -1 for each closed fermion loop, for each fermion line that both begins and ends in the initial state (i.e., $\bar{v} . . u$ ), and for each diagram in which fermion lines are interchanged in either of the initial or final states.

10. A particular useful spinor basis is given by

$$
u(\underline{k}, \lambda)=\frac{1}{\sqrt{k^{+}}}\left(k^{+}+\beta m+\vec{\alpha} \perp \cdot \vec{k}_{\perp}\right) \begin{cases}\chi(\uparrow) & \lambda=\uparrow \\ \chi(\downarrow) & \lambda=\downarrow\end{cases}
$$

or

$$
v(\underline{k}, \lambda)=\frac{1}{\sqrt{k^{+}}}\left(k^{+}-\beta m+\vec{\alpha} \perp \cdot \vec{k}_{\perp}\right) \begin{cases}\chi(\downarrow) & \lambda=\uparrow \\ x(\uparrow) & \lambda=\downarrow\end{cases}
$$

where $\chi(\uparrow)=1 / \sqrt{2}(1,0,1,0)$ and $\chi(\downarrow)=1 / \sqrt{2}(0,1,0,-1)^{T}$.

11. Color factors are computed as for covariant diagrams.

As an example we compute the magnetic formfactor $F_{2 l}$, associated with the decay of a heavy top quark $(t)$ into a massless bottom quark $(b)$ and a $W$-boson, via

$$
t \rightarrow b W
$$

to second-order in perturbation theory. In the limit of zero 6 mass the current associated with this process is given by

$$
\Gamma^{\mu}=\left(\gamma^{\mu} P_{L} F_{1 L}+i \sigma^{\mu \nu} q_{\nu} P_{R} F_{2 L}+q^{\mu} P_{R} C\right) b^{\dagger} t W^{\dagger}
$$

where the projection operators $P_{R, L}=(1 / 2)\left(1 \pm \gamma^{5}\right)$, the formfactors $F_{1 L}, F_{2 L}, C$ and the annihilation operators $b, t, W$ have been introduced. 


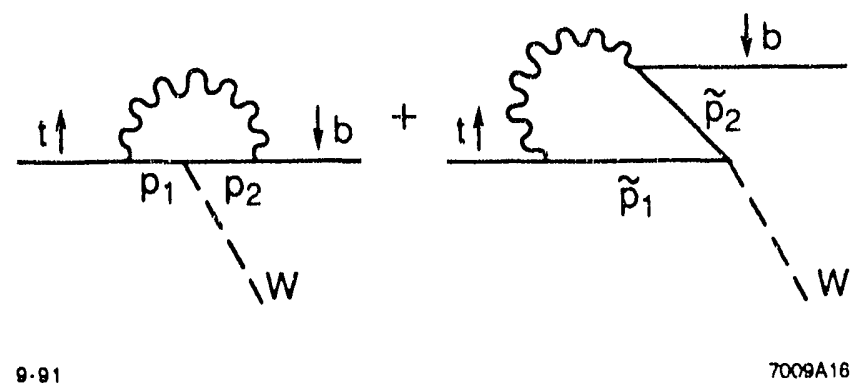

Figure 2.1. One-loop contribution to the decay $t \rightarrow b W$.

In order to extract $F_{2 L}$ it is convenient to consider the helicity-flip amplitude of the corresponding $\Gamma^{+}$current

$$
<\downarrow\left|\Gamma^{+}\right| \uparrow>=\left[-\left(p_{b}^{+}+p_{t}^{+}, F_{2 L}+\left(p_{b}^{+}-p_{t}^{+}\right) C\right] \bar{u}_{b}(\downarrow) u_{t}(\uparrow)\right.
$$

where $p_{t}=\left(p_{t}^{+}, p_{t}^{-}, p_{t, \perp}\right)$ and $p_{b}=\left(p_{b}^{+}, p_{b}^{-}, p_{b, \perp}\right)$ denote the four-momentum of $t$ (with mass $m$ ) and $b$ respectively. The l.h.s. of $\mathrm{E}_{\mathrm{l}}$. (2.6) can be computed in LCPTh, yielding two light-cone time-orderings shown in Fig. 2.1. Hence,

$$
\begin{aligned}
&<\bar{u}_{b}\left(p_{b}, \downarrow\right)\left|\Gamma^{+}\right| u_{t}\left(p_{t}, \uparrow\right)>= \\
& \frac{4}{3} \frac{e^{2}}{16 \pi^{3}} \int_{0}^{\infty} d^{2} k_{\perp} d k^{+} \frac{\Theta\left(p_{b}^{+}-k^{+}\right)}{k^{+}\left(p_{b}^{+}-k^{+}\right)\left(p_{t}^{+}-k^{+}\right)} \\
& \times \frac{\bar{u}_{b}(\downarrow) \gamma^{\mu} \not p_{2} \gamma^{+}\left(p_{1}+m\right) \gamma_{\mu} u_{t}(\uparrow)}{\left(p_{t}^{-}-\frac{m^{2}+p_{1, \perp}^{2}}{p_{1}^{+}}-\frac{k_{1}^{2}}{k^{+}}\right)\left(p_{b}^{-}-\frac{p_{2, \perp}^{2}}{p_{2}^{+}}-\frac{k^{2}}{\left.k^{+}\right)+i}\right.} \\
&+\frac{4}{3} \frac{e^{2}}{16 \pi^{3}} \int_{0}^{\infty} d^{2} k_{\perp} d k^{+} \frac{\Theta\left(k^{+}-p_{b}^{+}\right)}{k^{+}\left(k^{+}-p_{b}\right)\left(p_{t}^{+}-k^{+}\right)} \\
& \times \frac{\bar{u}_{b}(\downarrow) \gamma^{\mu}\left(-p_{2}\right) \gamma^{+}\left(p_{1}+m\right) \gamma_{\mu} u_{t}(\uparrow)}{\left(p_{t}^{-}-p_{b}^{-}-\frac{\tilde{p}_{2, \perp}^{2}}{\tilde{p}_{2}^{+}}-\frac{\tilde{p}_{1, \perp}^{2}+m^{2}}{\tilde{p}_{1}^{+}}\right)\left(p_{t}^{-}-\frac{\tilde{p}_{1, \perp}^{2}+m^{2}}{\tilde{p}_{1}^{+}}-\frac{k_{\perp}^{2}}{k^{+}}\right)+i c}
\end{aligned}
$$

where the four-vectors are given by $p_{1}=\left(p_{t}^{+}-k^{+}, p_{t}^{-}-k_{\perp}^{2} / k^{+}, p_{t, \perp}-k_{\perp}\right), p_{2}=$ $\left(p_{b}^{+}-k^{+}, p_{b}^{-}-k_{\perp}^{2} / k^{+}, p_{b, \perp}-k_{\perp}\right), \dot{p}_{1}=\left(p_{t}^{+}-k^{+},\left[\left(p_{t \perp}-k_{\perp}\right)^{2}+m^{2}\right] /\left(p_{t}^{+}-k^{+}\right), p_{1 \perp}-\right.$ 
$\left.k_{\perp}\right), \hat{p}_{2}=\left(k^{+}-p_{b}^{+}, p_{t}^{-}-p_{b}^{-}-\left(\tilde{p}_{1, \perp}-k_{\perp}\right)^{2} /\left(\tilde{p}_{1}^{+}-k^{+}\right), k_{\perp}-p_{b \perp}\right)$ and the factor $4 / 3$ includes color. Here, the momenta $p_{t}, p_{b}, k$ correspond to $t, b$ and the virtual gluon respectively. The computation of the r.h.s. of of $\mathrm{Eq} .(2.7)$ for two different. choices of the momentum $p_{b}$ (note that $\left(p_{t}-p_{b}\right)^{2}=m_{W}^{2}$, where $m_{W}$ is the $W$-boson mass) enables the extraction of $F_{2 L}$ in Eq. (2.6). For $m / m_{W}=150.0 / 80.9$ we find $F_{2 L}\left(m_{W}^{2}\right)=(0.49 \pm 0.001)\left(\alpha_{s} / 2 \pi\right)$.

Equation (2) in combination with Eq. (3) has the remarkable feature that they immediately lead to a practical prescription for the calculation of general scattering amplitudes in perturbation theory:

- Approximate the Hilbert space by a finite number of Fock states.

- Compute all matrix elements $\left\langle k_{i}|V| k_{j}\right\rangle$, between those Fock states $\left|k_{i}\right\rangle$.

- Connect initial and final state, insert the corresponding energy-denominators and sum over all intermediate states.

- Self-energy contributions can be identified by Fock states which occur more than once in the expansion. Thus, an appropriate rass subtraction seems possible. Wavefunction renormalization is not necessary since Fa. (2) corresponds to the summation of all Feyman diagrams to this order in perturbation theory. Hence, all wavefunction counterterms cancel by means of the Ward identitios. Charge renormalization can be carried out by identifying vacum polarization djagrams $[8]$.

Unfortunately, a direct numerical application of those steps is often extremely inefficient, since most of the Fock states, generally, do not connect initial and final states. In addition, ultraviolet regularization, by means of a Fock space truncation, poses extra problems. Nevertheless, due to the fact that the expansion in Eiq. (2) is manifestly unitary, i.e., causal, an efficient modification of the above procedure can be constructed for the automated computation of scattering amplitudes in perturbation theory. 


\section{REFERENCES for CHAPTER 2}

[1] G. P. Lepage and S. J.Brodsky, Phys. Rev, D22, 2157 (1980).

[2] S. J. Brodsky, private communication.

[3] For esthetic reasons [2] we want to keep the light-cone Hamiltonian in this formula. This is consistent if the total light-cone momentum $P^{+}$is set to one and the total perp. momentum is set to zero.

[4] G. Leibbrandt, Rev. Mod. Phys. 59, 1067(1987).

[5] A. Basseto, R. Soldati, Phys. Rev. D41, 3277 (1990).

[6] O. Piguet, G. Pollak, M. Schweda, Nucl. Phys. B328, 527 (1984).

[7] This rule will be derived in detail in the next chapter.

[8] In the case of non-Abelian gauge theories this is correct only if an axial gange is used. 


\section{Perturbation Theory in Light-Cone Quantization}

\subsection{A general algorithm for generating LCPTh}

In this section we develop a procedure which automatically constructs all lightcone time-orderings associated with a given Feynman diagram $F$. The only input required is the set of photon connections of $\mathrm{F}$ [first photon $(4,1)$, second photon $(5,2)$ in Fig. 3.1], which define the topology of the diagram.

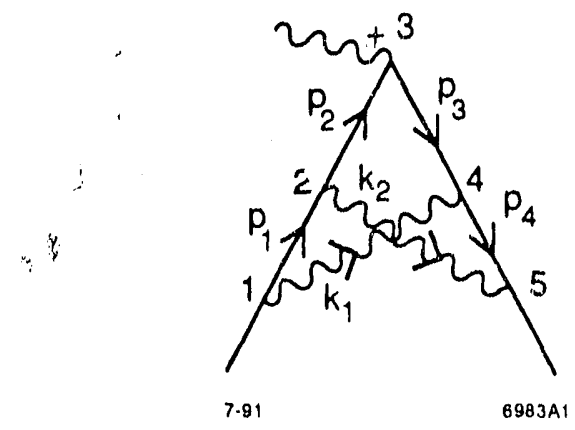

Figure 3.1. Two-loop QED cross diagram. The momenta $p_{i}$ and $k_{j}$ correspond to the internal momenta of the $\mathrm{i}$-th fermion and $\mathrm{j}$-th photon, respectively:

In the first part of this section we outline the procedure for quantum electrodynamics in the specific example of Fig. 3.1. In the remainder of the section a general algorithm, useful for higher loop calculations, is described.

First we shall review the derivation of LCPTh rules introduced by Soper [2]. The Feynman answer $\mathrm{F}$ for the two-loop contribution to the electromagnetic verlex $\gamma^{*}(q)+e_{1}^{-}\left(p_{I}\right) \rightarrow e_{\uparrow}^{-}\left(p_{F}\right)$ corresponding to Fig. 3.1 is given by $[1,7]$

$$
\begin{aligned}
F= & e^{4} \int d^{4} x_{1} d^{4} x_{2} d^{4} x_{3} d^{4} x_{4} d^{4} x_{5} \bar{\Psi}\left(x_{5}, \uparrow\right) \gamma^{\mu} \\
& \times i S_{F}\left(x_{5}-x_{4}\right) \gamma^{\lambda} i S_{F}\left(x_{4}-x_{3}\right) \frac{\gamma^{+} e^{-i q x_{3}}}{p_{1}^{+}} i S_{F}\left(x_{3}-x_{2}\right) \gamma^{\prime \prime} \\
& \left.\times i S_{F}\left(x_{2}-x_{1}\right)\right) \gamma^{\rho} \Psi\left(x_{1}, \downarrow\right) \quad i D_{F, \lambda \rho}\left(x_{4}-x_{1}\right) i D_{F, \mu \nu}\left(x_{5}-x_{2}\right),
\end{aligned}
$$


where $p_{I}^{+}$denotes the incoming light-cone momentum of the electron. Here we have chosen the helicity-flip amplitude $\left\langle p_{F}\left|J^{+} / p_{I}^{+}\right| p_{I}\right\rangle$ and the frame with $q^{+}=0$ which is appropriate for obtaining the anomalous magnetic moment of the electron and its Pauli form factor $F_{2}\left(q^{2}\right)[8]$. The Feynman propagator can be written in the convenient form [3]

$$
\begin{aligned}
S_{F}(x)= & \frac{-i}{(2 \pi)^{3}} \int d^{2} p_{\perp} \int_{0}^{\infty} \frac{d p^{+}}{p^{+}}\left(\Theta\left(x^{+}\right)(p+m) e^{-i p x}+\Theta\left(-x^{+}\right)(-p+m) e^{i p x}\right) \\
& +\frac{1}{(2 \pi)^{3}} \delta\left(x^{+}\right) \frac{1}{2} \gamma^{+} \int d^{2} p_{\perp} \int_{0}^{\infty} \frac{d p^{+}}{p^{+}} e^{-i\left(\frac{1}{2} p^{+} x^{-}-p_{\perp} x_{\perp}\right)} \\
= & S_{F}^{(+)}(x)+S_{F^{\prime}}^{(-)}(x)+S_{F}^{I n s t .}(x),
\end{aligned}
$$

where the electron four-vector is on the mass shell i.e. $p^{-}=\left(m^{2}+p_{\perp}^{2}\right) ! p^{+}$. This result follows from

$$
S_{F}(x)=\left(i \partial_{\mu} \gamma^{\mu}+m\right) \Delta_{F}(x)
$$

and

$$
\Delta_{F}(x)=\frac{-i}{(2 \pi)^{3}} \int d^{2} p_{\perp} \int_{0}^{\infty} \frac{d p^{+}}{p^{+}}\left(\Theta\left(x^{+}\right) e^{-i p x}+\Theta\left(-x^{+}\right) e^{i p x}\right) .
$$

The third term in Eq. (3.2) gives rise to an instantaneous fermion interaction in lightcone quantized QED. The photon propagator in light cone gauge $\eta \cdot A=A^{+}=0$ is given by

$$
\begin{aligned}
D_{\mu \nu}(x)= & \frac{-i}{(2 \pi)^{3}} \int d^{2} k_{\perp} \int_{0}^{\infty} \frac{d k^{+}}{k^{+}}\left(e^{-i k x} \Theta\left(x^{+}\right)+e^{i k x} \Theta\left(-x^{+}\right)\right) \sum_{\lambda} \epsilon_{\mu}^{*}(k, \lambda) \epsilon_{\nu}(k, \lambda) \\
& +\int d^{2} k_{\perp} \int_{0}^{\infty} d k^{+} \delta\left(x^{+}\right) \frac{2}{(2 \pi)^{3}} \delta_{\mu+} \delta_{\nu+\frac{1}{k^{+2}}} e^{-i\left(\frac{1}{2} k^{+} x^{-}-k_{\perp} x_{\perp}\right)} \\
\equiv & D_{\mu \nu}^{(+)}(x)+D_{\mu \nu}^{(-)}(x)+D_{\mu \nu}^{I n s t .}(x)
\end{aligned}
$$


where

$$
\sum_{\lambda=1,2} \epsilon_{\mu}^{*}(k, \lambda) \epsilon_{\nu}(k, \lambda)=-g_{\mu \nu}+\frac{\eta_{\mu} k_{\nu}+\eta_{\nu} k_{\mu}}{\eta k}
$$

This result can be obtained by performing the $k^{-}$integration of

$$
D_{\mu \nu}(x)=\frac{1}{(2 \pi)^{4}} \int d^{4} k e^{-i k x} \frac{-g_{\mu \nu}}{k^{2}+i \epsilon} .
$$

The external field $\Psi_{l}$ for the incident electron is given by

$$
\Psi_{I}(x)=u_{I}(p, s) e^{-i p_{I} x}
$$

where $u_{I}(p, s)$ is the solution of the free Dirac equation. In Feynman gauge the polarization sum $\sum_{\lambda=1,2} \epsilon_{\mu}^{*}(k, \lambda) \epsilon_{\nu}(k, \lambda)$ in $(3.4)$ gets replaced by $-g_{\mu \nu}$ and the instantaneous contribution drops out.

In order to compute the scattering amplitude, Eq. (3.1), using light-cone perturbation theory, one first has to split up the integration region into all possible time-orderings. For illustration purposes we pick a typical time-ordering $\tau_{14325}$

$$
x_{1}^{+}<x_{4}^{+}<x_{3}^{+}<x_{2}^{+}<x_{5}^{+}
$$

and obtain the contribution

$$
\begin{aligned}
F_{(14325)}^{(r)}= & e^{4} \int d^{4} x_{1} d^{4} x_{2} d^{4} x_{3} d^{4} x_{4} d^{4} x_{5} \\
& \times \Theta\left(x_{4}^{+}-x_{1}^{+}\right) \Theta\left(x_{3}^{+}-x_{4}^{+}\right) \Theta\left(x_{2}^{+}-x_{3}^{+}\right) \Theta\left(x_{5}^{+}-x_{2}^{+}\right) e^{+i p_{F} x_{5}} \bar{u}\left(p_{F}, \uparrow\right) \gamma^{\prime \prime} \\
& \times i S_{F}^{(+)}\left(x_{5}-x_{4}\right) \gamma^{\lambda} i S_{F}^{(-)}\left(x_{4}-x_{3}\right) \frac{\gamma^{+} e^{-i q x_{3}}}{p_{I}^{+}} i S_{F}^{(-)}\left(x_{3}-x_{2}\right) \gamma^{\prime \prime} \\
& \times i S_{F}^{(+)}\left(x_{2}-x_{1}\right)\left(\gamma^{\rho} e^{-i p_{I} x_{1}} u\left(p_{I}, \downarrow\right)\right) i D_{F, \lambda \rho}^{(+)}\left(x_{4}-x_{1}\right) i D_{F, \mu \nu}^{(+)}\left(x_{5}-x_{2}\right)
\end{aligned}
$$

The corresponding $\tau$-ordered diagram is shown in Fig. 3.2. Note that the instantaneous contributions in $S_{F}\left(x_{4}-x_{3}\right)$ and $S_{F}\left(x_{3}-x_{2}\right)$ do not contribute because of 


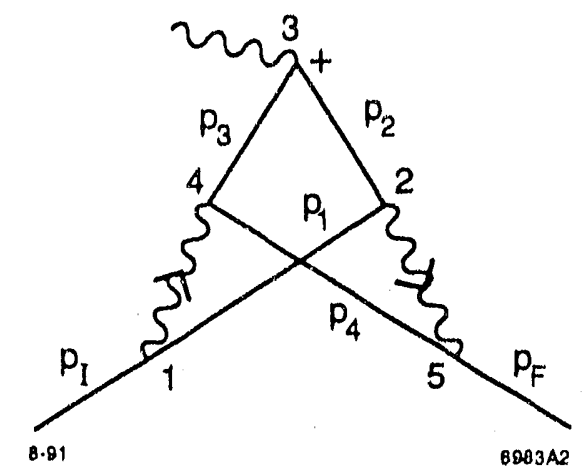

Figure 3.2. Light-cone time-ordering contributing to the cross diagram.

$\gamma^{+} \gamma^{+}=0$. The instantaneous contribution of $S_{F}\left(x_{2}-x_{1}\right)$ gives zero result in this case due to

$$
\delta\left(x_{2}^{+}-x_{1}^{+}\right) \Theta\left(x_{4}^{+}-x_{1}^{+}\right) \Theta\left(x_{3}^{+}-x_{4}^{+}\right) \Theta\left(x_{2}^{+}-x_{3}^{+}\right) \Theta\left(x_{5}^{+}-x_{2}^{+}\right) \equiv 0 .
$$

In the same way it is shown that the instantaneous contribution of $S_{F}\left(x_{5}-x_{4}\right)$ vanishes. In general, instantaneous interactions give rise to a nonzero contribution only if they do not extend over more than one intermediate state for the same reason [9] . Equation (3.8) leads to a phase factor of the form

$$
\begin{aligned}
& \exp \left\{i \left[p_{F} x_{5}-p_{4}\left(x_{5}-x_{4}\right)+p_{3}\left(x_{4}-x_{3}\right)+p_{2}\left(x_{3}-x_{2}\right)\right.\right. \\
& \left.\left.-q x_{3}-p_{1}\left(x_{2}-x_{1}\right)-p_{1} x_{1}-k_{1}\left(x_{4}-x_{1}\right)-k_{2}\left(x_{5}-x_{2}\right)\right]\right\} .
\end{aligned}
$$

The momenta $p_{i}, k_{j}$ denote the momentum associated with the $\mathrm{i}$-th fermion and the $\mathrm{j}$-th photon line respectively. The momenta $p_{I}, p_{F}$ corresponds to the initial and final momentum respectively. The integration over $x_{i}^{+}, x_{i}^{-}$can be performed trivially and demonstrates momentum conservation of $p_{i}^{+}, p_{i}^{+}$at each vertex. 
In order to perform the $x_{i}^{+}$integration, it is convenient to change variables according to [2]

$$
\begin{aligned}
& \lambda_{1}^{+}=x_{4}^{+}-x_{1}^{+} \\
& \lambda_{2}^{+}=x_{3}^{+}-x_{4}^{+} \\
& \lambda_{3}^{+}=x_{2}^{+}-x_{3}^{+} \\
& \lambda_{4}^{+}=x_{5}^{+}-x_{2}^{+} .
\end{aligned}
$$

The light-cone time part of (3.9) becomes

$$
\begin{aligned}
& \exp \frac{1}{2}\left[i \lambda_{4}^{+}\left(p_{4}^{-}-k_{2}^{-}-p_{F}^{-}\right)+i \lambda_{3}^{+}\left(-p_{2}^{-}-p_{1}^{-}-p_{4}^{-}+p_{F}^{-}\right)\right. \\
& +i \lambda_{2}^{+}\left(-p_{3}^{-}-p_{1}^{-}-p_{4}^{-}+p_{F}^{-}-q^{-}\right) \\
& \left.+i \lambda_{1}^{+}\left(-k_{1}^{-}-p_{1}^{-}+p_{F}^{-}-q^{-}\right)+i x_{1}^{+}\left(-p_{I}^{-}-q^{-}+p_{F}^{-}\right)\right] \\
& =\exp \frac{1}{2}\left[i C_{F}\left(\tau_{14325}\right)+i x_{1}^{+}\left(-p_{I}^{-}-q^{-}+p_{F}^{-}\right)\right],
\end{aligned}
$$

This will play an important role in our discussion, so we have introduced the definition of a characteristic exponent $C_{F}(\tau)$ of a time-ordering $\tau$. The integral over $x_{1}^{+}$can be performed trivially and gives overall light-cone energy conservation. The remaining integrals over $x_{i}^{+}$can be performed by means of

$$
\int_{0}^{\infty} d T e^{i(H+i c) T}=\frac{i}{H+i t} .
$$

The product of these denominators, and the factors $\left[-i /(2 \pi)^{3}\right]\left(1 / p^{+}\right)$from $(3.2)$ and (3.4) then lead to the LCPTh answer of the time-ordering (3.7).

As far as the treatment of instantaneous diagrams is concerned, a simple sub. stitution allows the incorporation of instantaneous vertices [9]. 'To see this, consider the $\gamma^{+}$contribution of one fermion line to an arbitrary Feynman diagram

$$
F=\ldots\left(\frac{\frac{1}{2} \gamma^{+} p^{-}}{p^{+} d^{-}}+\frac{\gamma^{+}}{2 p^{+}}\right) \ldots
$$


where $d^{-}=p_{\bar{I}}^{-}-p^{-}-\sum_{\text {spec }} p_{i}^{-}$is the light-cone denominator containing the fermion line under consideration. In general, $p_{I}^{-}$is given by the total light-cone energy of the incoming particles and the sum runs over all spectators of the corresponding intermediate state.

The second term in Eq. (3.12) presents the instantaneous contribution to $F^{\prime}$. If we define $p_{\text {energy-shell }}^{-}=p^{-}+d^{-}$, both terms combine to

$$
F=\ldots \frac{\frac{1}{2} \gamma^{+} p_{\text {energy-shell }}^{-}}{p^{+} d^{-}} \ldots
$$

Note that $p_{\text {energy-shell }}^{-}$is the light-cone energy one would obtain if one required lightcone energy conservation at the vertex. Thus all instantaneons fermion contributions can be taken into account by putting those $p^{-}$on energy-shell in the numerator whenever that fermion does not extend over more than one intermediate state [9]. In the same way the light-cone gauge photon interaction in (3.4) can be handled [A].

Now we are ready to describe our general as a sequence of 10 steps (sec Fig. 3.3). For illustration we again consider the order $e^{5}$ contribution to the electron vertex. We start out noting that each two-loop $\tau$-ordered contribution to the electron vertex (which contains no vacuum polarization contribution $[10]$ ) is of the form

$$
\begin{aligned}
F\left(i_{1}, \ldots, i_{5}\right)= & \frac{e^{4}}{\left(16 \pi^{3}\right)^{2}} \int \frac{d k_{1}^{+} d^{2} k_{1} d k_{2}^{+} d^{2} k_{2}}{p_{1}^{+} p_{2}^{+} p_{3}^{+} p_{4}^{+} k_{1}^{+} k_{2}^{+}} \frac{\Theta\left(p_{1}^{+}\right) \Theta\left(p_{2}^{+}\right) \Theta\left(p_{3}^{+}\right) \Theta\left(p_{4}^{+}\right)}{d^{-}(1) d^{-}(2) d^{-}(3) d^{-}(4)} \\
& \times\left(\bar{u} \gamma^{\mu\left(i_{1}\right)}\left(s_{4} p_{4}+m\right) \gamma^{\mu\left(i_{2}\right)}\left(s_{3} p_{3}+m\right) \gamma^{\mu\left(i_{3}\right)}\left(s_{2} p_{2}+m\right) \gamma^{\mu\left(i_{4}\right)}\right. \\
& \left.\times\left(s_{1} p_{1}+m\right) \gamma^{\mu\left(i_{5}\right)} u\right) \quad D_{\mu(1) \mu(2)}\left(k_{1}\right) D_{\mu(4) \mu(5)}\left(k_{2}\right)
\end{aligned}
$$

where the diagram is defined by its photon connections. The explicit construction of $(3.14)$ is done as follows:

- Step (I): the indices $i_{1}, i_{2}, \ldots, i_{5}$ are specified. For the diagram of Fig. 3.1 we have $i_{1}=5, i_{2}=2, i_{3}=+, i_{4}=4, i_{5}=1$. For the diagram of Fig. 3.4 we have $i_{1}=5, i_{2}=+, i_{3}=2, i_{4}=4, i_{5}=1$. 


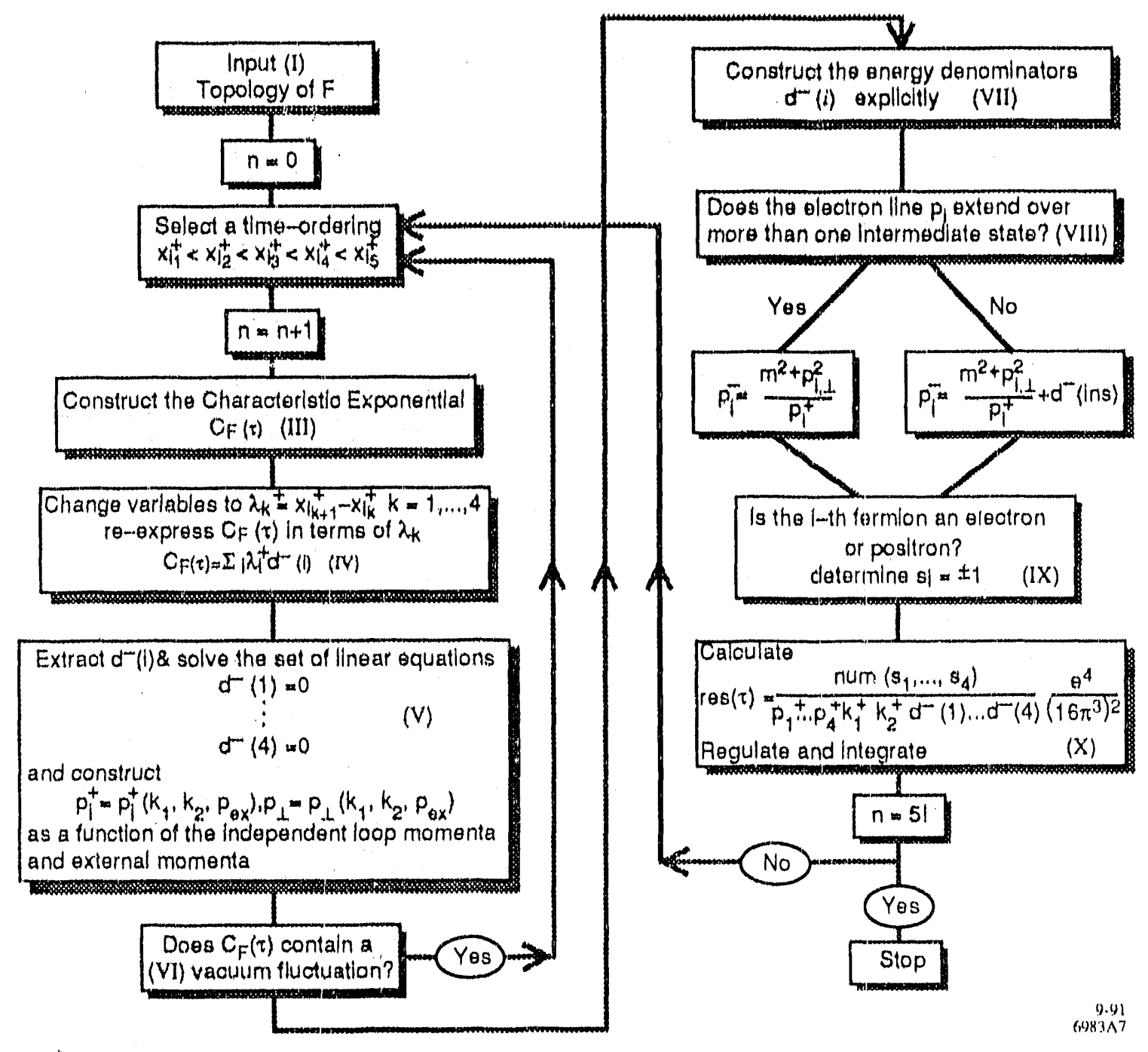

Figure 3.3. Flow chart for the automatic computation of (QED) amplitudes.

- Step (II): For each of the $n !=120$ time-orderings one defines a vector $\tau(I), i=$ $1, \ldots, n=5 . \tau(I)$ describes the position of the I-th vertex of $F$. In the example: of Fig. 3.1 we get $\tau(1)=1, \tau(2)=2, \tau(3)=3, \tau(4)=4, \tau(5)=5$. In the example of Fig. 3.2 we get $\tau(1)=1, \tau(2)=4, \tau(3)=3, \tau(4)=2, \tau(5)=5$. It is also useful to define

$$
A(\tau(I))=I
$$




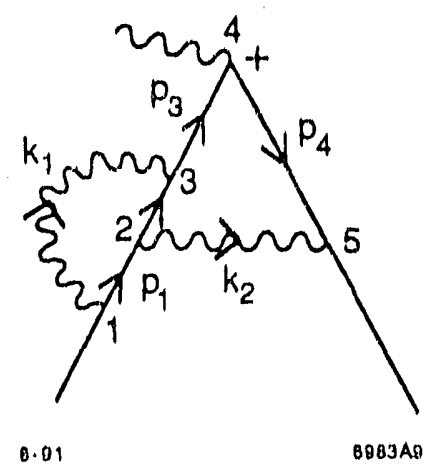

Figure 3.4. "Two-loop "comer" diagram.

- Step (III): Once a time-ordering is defined we know which pieces ont of the propagators (3.2) and (3.4) are to be picked. The construction of ( $F(\tau)$ de. fined in (3.11) is straightforward. Note that the term which describes overall monentum conservation must be subtracted in order to obtain $\left({ }_{F}(T)\right.$.

- Step (IV): one changes variables to $\lambda_{k}^{+}=r_{i_{k+1}^{+}}^{+}-r_{i k}^{+}$for $k=1, \ldots, 4$ and expresses the characteristic exponential in terms of $\lambda_{k}$ :

$$
C_{F}(\tau)=\sum_{i} \lambda_{i}^{+} d^{-}(i)
$$

- Step (V): In general 5! different time-orderings can contribute to F. However. in practice most of them vanish. This is due to the fact that all light-cone momenta are greater or equal zero and conserved at each vertex [11]. An example is given in Fig. 3.5, which contains a vacuum fluctuation at $r_{4}$. A vacuum fluctuation at $x_{i}$ can be formally identified when all terms of $d^{-}(i)$ carry the same coefficient (namely, +1 , or -1 ). The $d^{-(i)}$ can be obtinined from $C_{F}(\tau)$ by setting $\lambda_{k}=\delta_{k i}$.

- Step (VI): To obtain the form Eq. (3.14) all momenta $p_{i}^{+}$and $\eta_{1}^{+}$must he expressed in terms of the independent loop momenta and external momentan. This can be achieved by solving the equations 


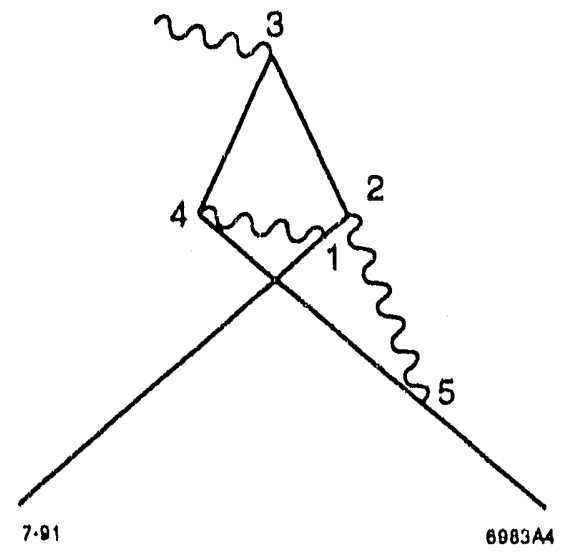

Figure 3.5. Example for a vacuum fluctuation to the cross diagram.

$$
\begin{aligned}
& d^{-}(1)=0, \\
& d^{-}(2)=0 \\
& d^{-}(3)=0 \\
& d^{-}(4)=0
\end{aligned}
$$

For example, for Fig. 3.2 we find

$$
\begin{aligned}
p_{1}^{+}=p_{I}^{+}-k_{1}^{+}, & p_{1 \perp}=p_{I \perp}-k_{1 \perp} \\
p_{2}^{+}=k_{1}^{+}+k_{2}^{+}-p_{I .}^{+}, & p_{2 \perp}=k_{1 \perp}+k_{2 \perp}-p_{I \perp} \\
p_{3,}^{+}=k_{2}^{+}+k_{1}^{+}-p_{F}^{+}, & p_{3 \perp}=k_{2 \perp}+k_{1 \perp}-p_{r \perp} \\
p_{4}^{+}=-k_{2}^{+}+p_{F}^{+}, & p_{4 \perp}=-k_{2 \perp}+p_{F \perp} .
\end{aligned}
$$

- Step (VII): The expressions for the internal fermion momentin, obtained in step (VI), are substituted into $d^{-}(i)$ in order to construct all energy denominators $d^{-}(i)$ explicitly. 
- Step (VIII): When setting up the fermion $p_{i}^{-}$in the numerator it must be decided whether the fermion line $p_{i}$ extends over more than ofre intermediate state. A formal criteria for that is given by

$$
\operatorname{abs}(A(i)-A(i+1))=1
$$

If (3.18) is correct, $p_{i}^{-}$is set on energy shell, which means

$$
p_{i}^{-}=p_{i, \text { mass-shell }}+d^{-}(\text {ins } s)
$$

where ins $:=\min (A(i+1), A(i)), p_{i, \text { mats }- \text { shell }}^{-}=\left(m^{2}+p_{i \perp}^{2}\right) / p_{i}^{+}$. If $(3.18)$ is not fulfilled we have $p_{i}^{-}=p_{i, \text { mass }- \text { shell }}^{-}$

- Step (IX). The only variables which are left to be determined in (3.14) are the signs $s_{i}$ which define whether a fermion or antifermion propagates. The phase can be determined from

$$
s_{i}=\frac{A\left(x_{i+1}\right)-A\left(x_{i}\right)}{a b s\left(A\left(x_{i+1}\right)-A\left(x_{i}\right)\right)}
$$

- Step (X): (3.14) can now be calculated. If necessary, the diagran can be regularized using Pauli--Villars regularization.

It should be noted that step 2 to step 10 can be readily carried out automatically, using an algebraic manipulation program like REIUUCE. The algorithm can be generalized easily to higher loops. As an example, in Fig. 3.6 we present the time-orderings, generated by the algorithm, to a three-loop contribution of the clectromagnetic vertex for $q^{+}=0$. 

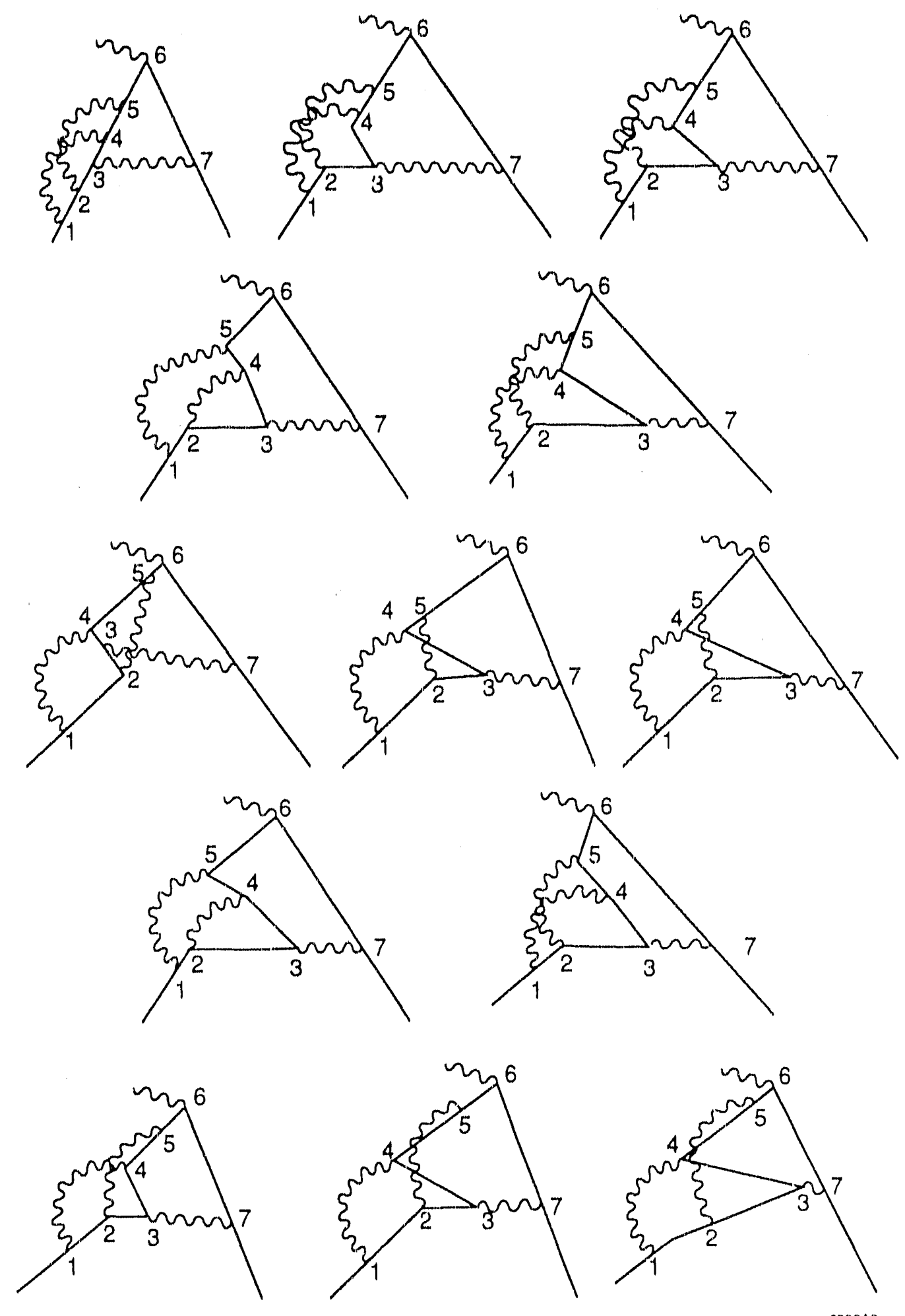

Figure 3.6. Example for a set of light-cone time-orderings which correspond to a sixth order Feynman diagram. 


\subsection{Numerical results}

In this section we report on the use of the general LCP'Th algorithm to redo the two-loop calculation of the anomalous magnetic moment $(g-2) / 2=a=F_{2}(0)$ by Petermann and Sommerfield [12,13]. Figure 3.7 shows all Feynman diagrams as well as the corresponding light-cone time-orderings, contributing to the anomaly in fourth order.

The vacuum polarization diagram 6 can be computed by the effective replacement [14]

$$
\frac{1}{h^{2}-\lambda^{2}+i c} \rightarrow \frac{\alpha}{\pi} \int_{0}^{1} d t \frac{t^{2}\left(1-\frac{1}{3} t^{2}\right)}{1-t^{2}} \frac{1}{k^{2}-\frac{4 m m^{2}}{1-t^{2}}+i c}
$$

after performing the integration over $k^{-}[15,16]$. All diagrams in Fig. 3.7 (with cxception of graph 5) are ultraviolet divergent and require renormalization. However. by computing certain sets of diagrams simultaneously, the calculation can be arranged such that ultraviolet divergences cancel between diagrams of the same set. As an example, Table 3.1 shows the result of the numerical integration, using the adaptive integration routine VEGAS [17] of diagram 1 and 2 for different values for the ultraviolet cut off $\Lambda^{2}$. After mass renormalization of the self-energy diagran 2 , we observe only a residual $\Lambda$ dependence of the form

$$
\frac{1}{\Lambda^{2}} \operatorname{lng} \Lambda^{2}
$$

which can be easily eliminated by an appropriate fit in $\Lambda^{2}[18]$.

We obtain for our estimate of diagrams 1 and $2, a=(-0.326 \pm 0.001)\left(\alpha^{2} / \pi^{2}\right)$, which is to be compared with the analytic answer of Petermann [19] $a=-0.327 \ldots$ $\alpha^{2} / \pi^{2}$ and Sommerfield. Table 3.2 shows the result of the residual diagrams. The agreement with the correct result is better than $0.2 \%$ for single diagrams. To obtain 


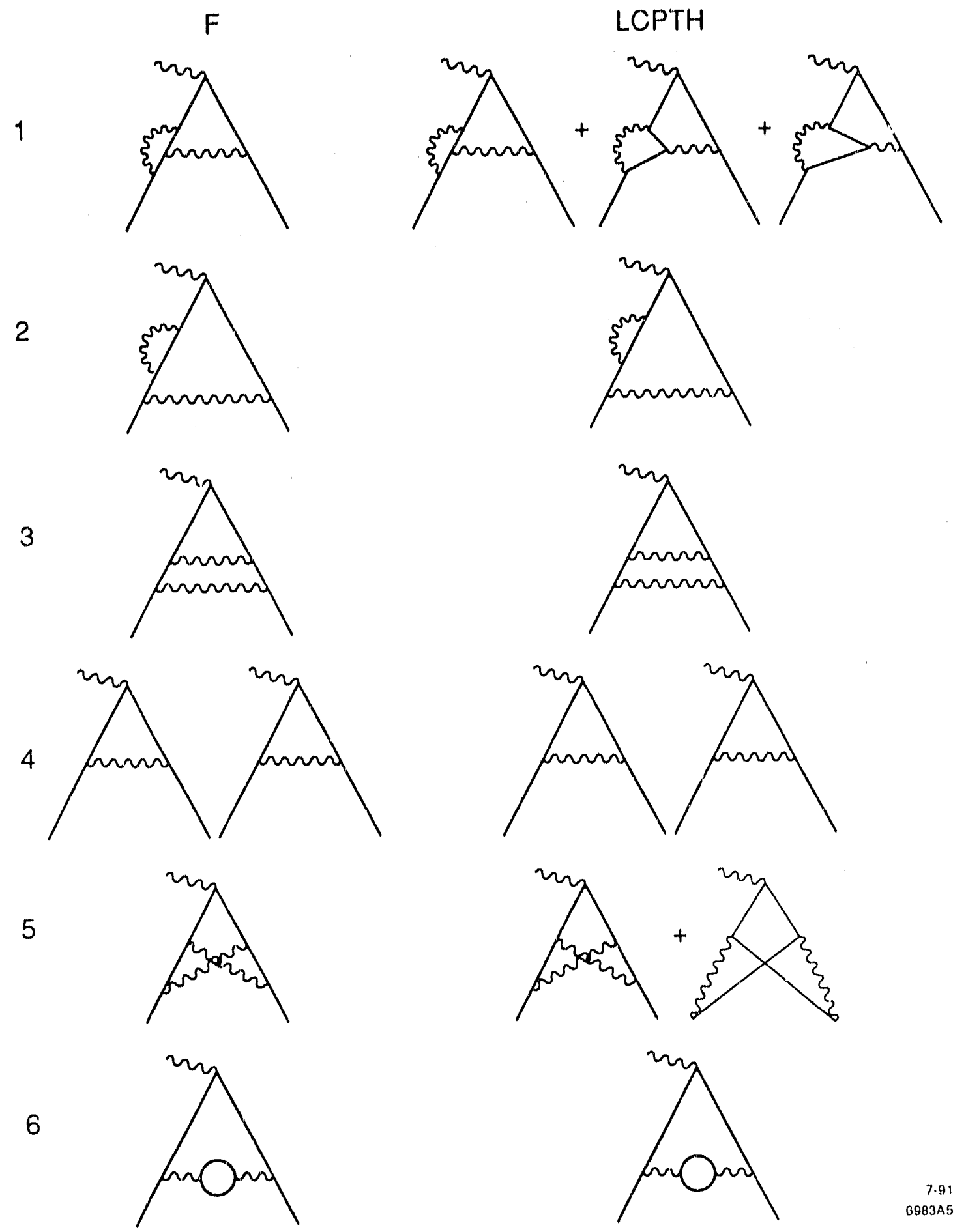

Figure 3.7. Six Feynman diagrams and the corresponding light-cone time-orderings contributing to the fourth-order anomalous magnetic moment to the electron.

20 
Table 3.1. Result $(\delta a)$ of the numerical integration for diagram $1+2$ after mass renormalization in units of $(\alpha / \pi)^{2}$. The data converge for large values of the Pauli-Villars cut-off $\Lambda$ (in units of the electron mass).

\begin{tabular}{|c|c|}
\hline$\delta \mathrm{a}$ & Lambda \\
\hline-0.289 & 25 \\
-0.305 & 50 \\
-0.313 & 100 \\
-0.324 & 1000 \\
\hline
\end{tabular}

Table 3.2. Numerical results $(\delta a)$ for the two-loop diagrams of Fig. 3.7. The results are compared with the analytic answer by Petermann.

\begin{tabular}{|c|c|c|}
\hline Diagram & $\delta a$ & Result by Petermann \\
\hline $1+2$ & $-0.326 \pm 0.001$ & -0.327 \\
$3+4$ & $0.780 \pm 0.007$ & 0.778 \\
5 & $-0.465 \pm 0.002$ & -0.467 \\
6 & $0.016 \pm 0.001$ & 0.016 \\
\hline
\end{tabular}

Table 3.3. Numerical results $(\delta a)$ for the sixth-order contributions of the diagrams in Fig. 3.8. The results are compared with the results given by Brodsky and Kinoshita.

\begin{tabular}{|c|c|c|}
\hline Diagram & $\delta a$ & Result by Brodsky and Kinoshita \\
\hline $4+7$ & $-0.114 \pm 0.002$ & -0.115 \\
$1+2$ & $-0.0031 \pm 0.003$ & -0.0032 \\
$5+6$ & $0.053 \pm 0.002$ & 0.053 \\
$3+8$ & $-0.09 \pm 0.02$ & -0.088 \\
\hline
\end{tabular}

these results we needed typically only one minute of CPU per graph on a IBMB3090. These successes encouraged us to attempt some sixth order moment calculations for the Feymman graphs shown in Fig. 3.8. n Table 3.3 we compare our estinute with the results obtained by Brodsky and Kinoshita [20]. For further references ser also [21]. 
1

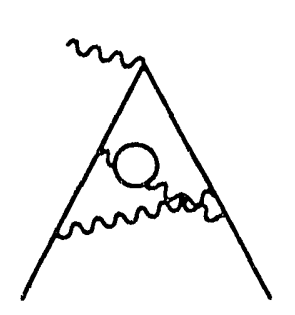

2

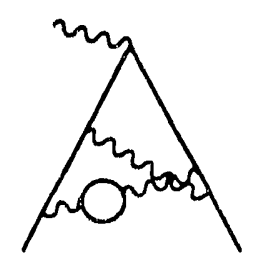

3
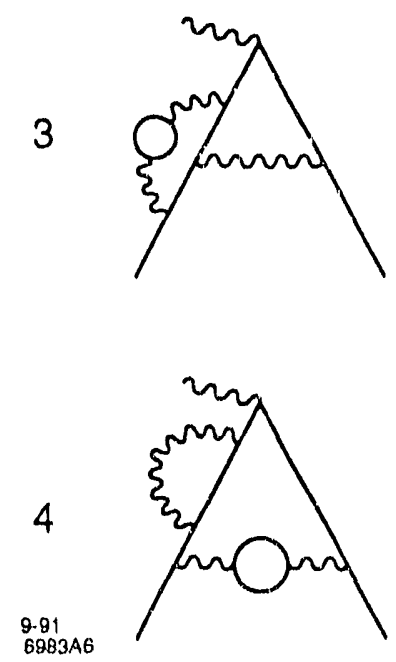

$\mathrm{F}$

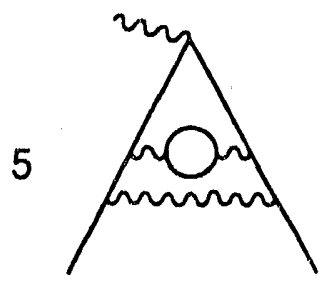

6
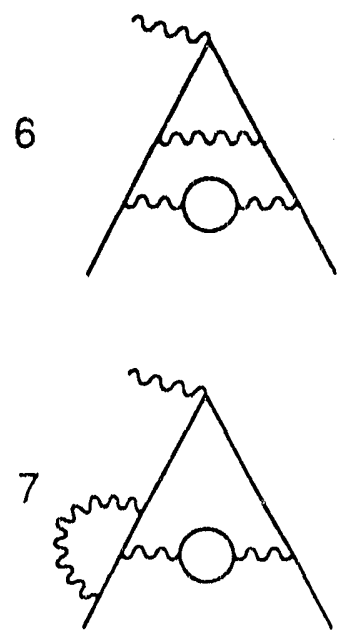

8

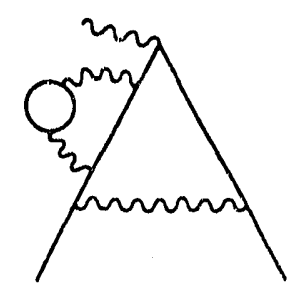

Figure 3.8. Sixth-order Feynman diagrams containing one-loop vacuum polarization.

\subsection{Summary}

We have presented a new algorithm for the automatic computation of Feymman diagram amplitudes. The method, which is based on light-cone perturbation theory (LCPTh), is explored for two- and three-loop calculations in QED. The amplitudes are constructed automatically and explicitly, given just the photon connections of the corresponding diagrams. The extension of the algorithm to higher loops is straightforward [22]. 
One of the most useful applications of LCPTh and this algorithm could be the computation of multi-jet processes in $e^{+} e^{-}-$annihilation, since LCPTh amplitudes correspond closely to the quark and gluon jets identified in high energy physics. These reactions have not been completely calculated beyond the one-loop order in perturbation theory. However, the extension to quantum chromodynamics requires a more careful regularization of the ultraviolet behavior of the theory. The implementation of dimensional regularization and other renormalization issues will be described in Chapter 4. 


\section{REFERENCES for CHAPTER 3}

[1] More precisely the spin-flip amplitude of the $\gamma^{+}$current with momentum transfer $\left(0,0, q_{x}, q_{y}\right)$ projects out $\left(q_{x}+i q_{y}\right) F_{2}(0)$.

[2] David E. Soper, SLAC-PUB-0137 (1971).

[3] John B. Kogut, Davison E. Soper, Phys. Rev. D1, 2901 (1970).

[4] G. P. Lepage and S. J. Brodsky, Phys. Rev. D22, 2157 (1980).

[5] Y. Frishman, Phys. Reports 13C, 1 (1974).

[6] J. D. Bjorken, J. B. Kogut and D. E. Soper, Phys. Rev. D3, 1382 (1971).

[7] S. D. Drell, D. J. Levy, and T. M. Yan, Phys. Rev. Lett. 22, 744 (1969); Phy's. Rev. 187, 2159 (1969).

[8] S. J. Brodsky, S. D. Drell, Phys. Rev. D22, 2236 (1980)

[9] S. J. Brodsky, R. Roskies and R. Suaya, Phys. Rev. D8, 1574 (1973).

[10] The vacuum polarization diagram can be taken into account by a similat expression to Eq. (3.14) (at one loop) where the photon propagation is replaced by an effective one loop propagator. We will discuss this point in the next section.

[11] This is one of the main advantages of $x^{+}$-ordered perturbation theory.

[12] A. Petermann, Helv. Phys. Acta 30, 407 (1957)

[13] C. M. Sommerfield, Ann. Phys. (NY) 5, 26 (1958).

[14] G. Kallen, Hel. Phys. Acta 25, 417 (1952).

[15] This is why only one time-ordering appears for this graph.

[16] The same can be done for two loop vacuum polarization insertions.

[17] G. P. Lepage, J. Comput. Phys. D27, 192 (1978). 
[18] The mass renormalization can be performed in this case by the method of alternate denominators [9]. Whenever one Fock state appears twice in a diagram, the total energy which appears in the denominator(s) of the embedded self-energy must be replaced by the total energy adjacent to this self-energy correction In order to keep the discussion as simple as possible, we have not included this step into the logic of Fig. 3.5. However, this can be done in a straightforward way, when ultraviolet regularization is needed.

[19] A. Petermann, Helv. Phys. Acta 30, 407 (1957).

[20] S. Brodsky and T. Kinoshita, Phys. Rev. D3, 356 (1971).

[21] T. Kinoshita, B. Nizic, Y. Okamoto, Phys. Rev D41, 593 (1990).

[22] However, the numerical effort, necessary to obtain stable and reliable results, typically increases by one order of magnitude, at each increasing order in perturbation theory. 


\section{Ultraviolet Regularization of Light-Cone Hamiltonian Perturbation Theory: Application to the Anomalous Magnetic Moment of the Electron in Light-Cone Gauge}

\subsection{Introduction}

One objective of this chapter is to explore some of the renormalization issues in the example of the anomalous magnetic moment of the electron $a=(g-2) / 2$ to order $(\alpha / \pi)^{2}$. In particular, the discussion shall focus on a renomalization schene which is also suitable for a numerical treatment. This requires the construction of certain counterterms on the local level in order to prevent round off errors.

The first section of this chapter addresses problems associated with quadratio divergences in light-cone quantized gange theories. It is shown that Feymman gange leads to an infinite number of quadratic divergent LC.P'Th diagrams at one loop. The situation is significantly better in light-cone gauge since in the continumm only the self-energy and the vacuum polarization display a culadratic divergence at one loop. However, a computation in $A^{+}=0$ gange requares a careful regularization of the associated gauge singularity. Most regulators reduce the small re behavion of the light-cone photon propagator to that present in Feynman gauge. Thus, an understanding of Fynman gauge is essential even if calculations are carriced oul in $A^{+}=0$ gauge.

The second section discusses the fourth-order correction of $g-2$ in the light cone gauge. Two different descriptions for the regularization of the kit singularity are dis. cussed. The sensitivity of physical observables to a finite truncation is investigated.

'The third section tests the ultraviolet regulators, which are commonly used fol' the purpose of nonperturbative calculations in DLCQQ. It is shown that these reg. 
ulators do not recover the correct answer for $a=(g-2) / 2$ in fourth order, unless special counterterms are invoked.

\subsection{Light-cone quantization in Feynman gauge}

In any gauge different from light-cone gauge, canonical light-cone quantization is anything but straightforward. 'This is due to the fact that, after solving the spinor' constraint equation, the light-cone Hamiltonian in these gauges contains terms which are of arbitrarily high order in the $A^{+}$field. Thus, in this case, we will not attempt. to write down the light-cone Hamiltonian. However, even without constructing the light-cone Hamiltonian explicitly, one can still derive light-cone perturbation theory (LCPTh) rules for Feyman gauge simply by separating the various light-cone timeorderings of the Feymman amplitudes. A useful reference can be found in [1] (see also Chapter 1).

Feynman perturbation theory in Feynman gange has the advantage that even uffshell Greens' functions exhibit the full Lorentz structure. This simple foature provides important consistency checks for light-cone quantized field theories, since manifrst covariance is lost in this case. In addition, it helps to disentangle problems associated with singularities in the light-cone gauge propagator from problems intrinsic to lightcone quantization itself.

We start our discussion with the evaluation of the fourth order correction to the anomalous magnetic moment of the electron $(g-2) / 2$ in LCPTh. The Fegnman diagrams and the corresponding light-cone time-orderings are displayed in Fig. 4.1. 'The' techniques we used for this calculation has been discussed in the previous chapter. so that we only compare the LCP'Th answer of the anomaly

$$
a_{L C P T h}=(-0.324 \pm 0.004) \frac{\alpha^{2}}{\pi^{2}}
$$


1
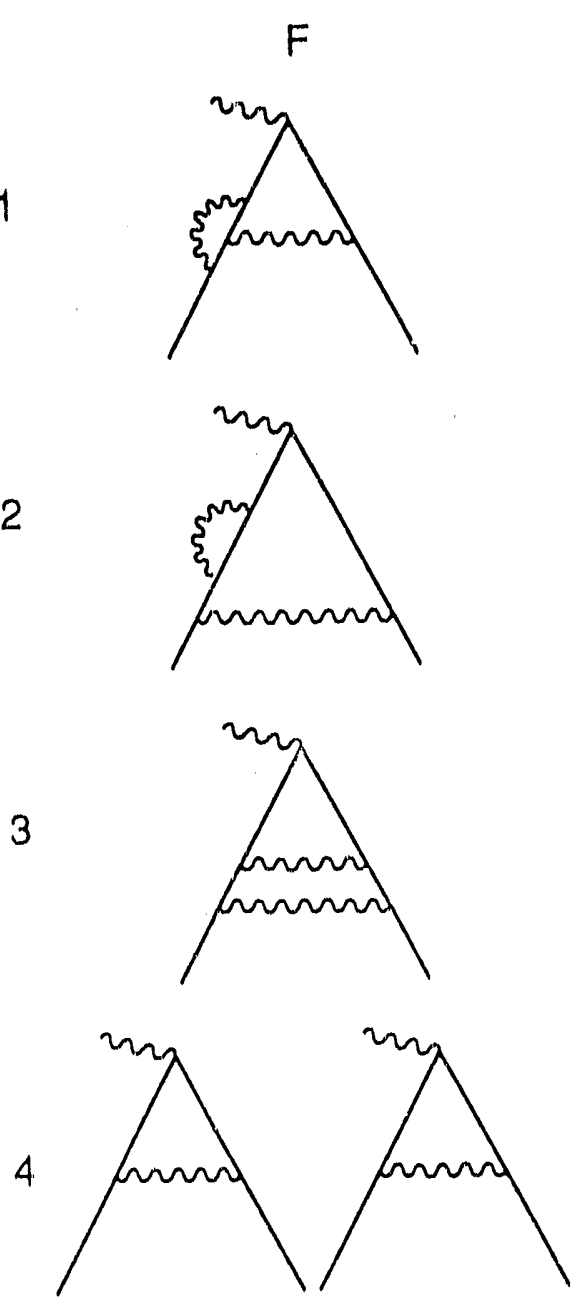

5

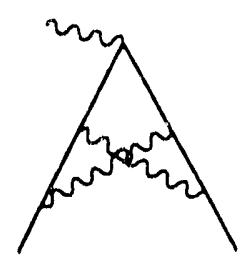

6

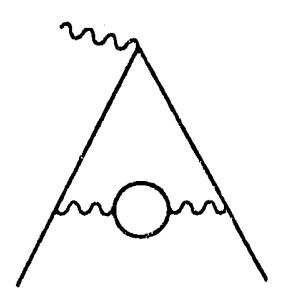

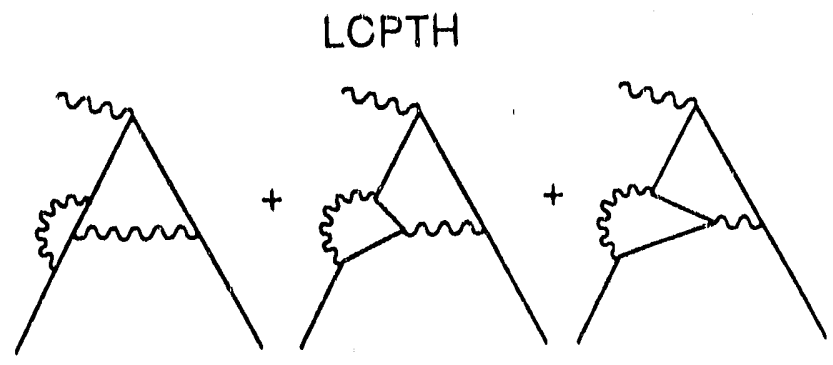
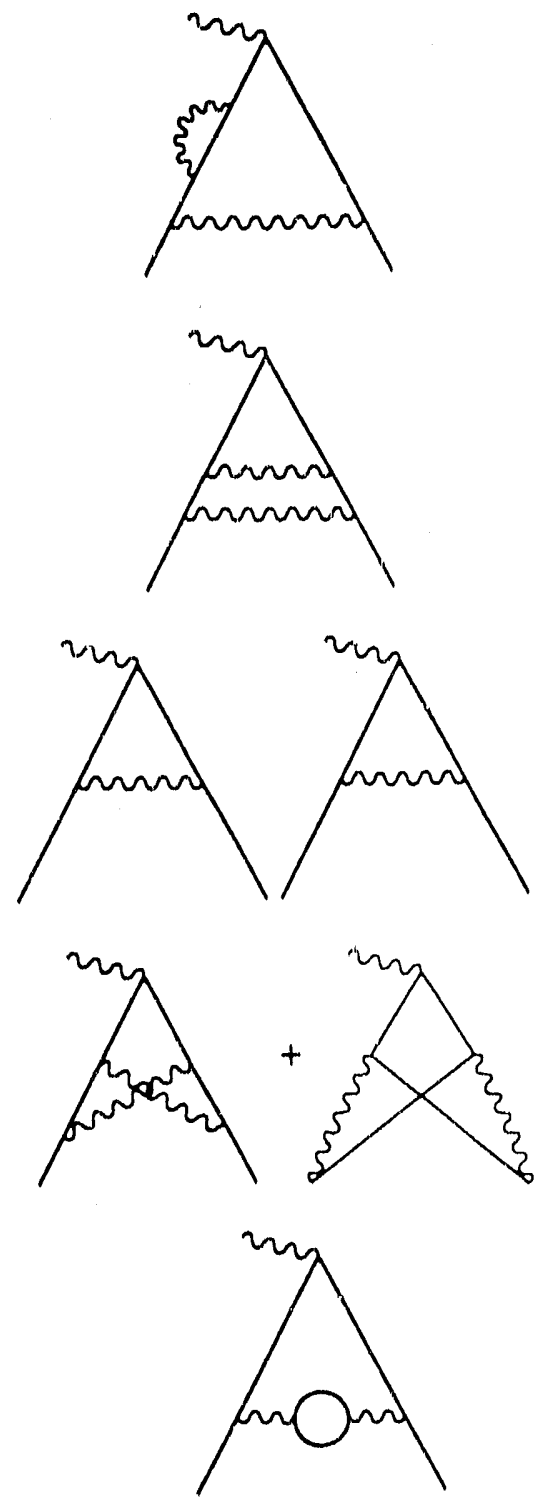

7.91

Figure 4.1. Feynman diagrams $F$ with corresponding light-cone time-ordered diagram contributing to the electron anomalous magnetic moment $a=(g-2) / 2$ to fourth order.

36 


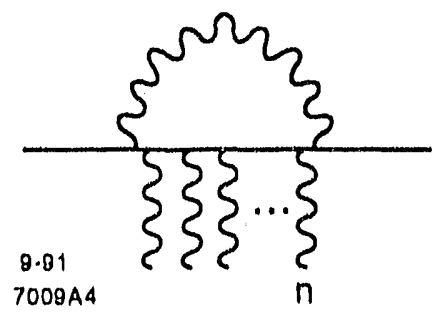

Figure 4.2. n-photon jellyfish graph.

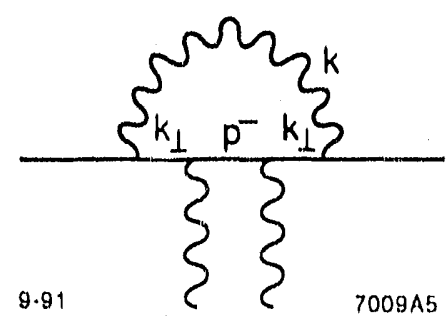

Figure 4.3. Powercounting for the $n=2$ jellyfish diagram leads to a logarithuic divergence.

with the analytic result by Petermann and Sommerfield $[2,3]$

$$
a=-0.32 \uparrow \ldots \frac{\alpha^{2}}{\pi^{2}}
$$

In Chapter 3 some sixth order contributions have also been calculated using LCP'Th. It should be emphasized that, in order to obtain this agrement, additional renormalization, beyond usual procedures, is necessary for the self-cuergy diagram 2 in Fig. 4.1. This is because the one-loop self-energy exhibits a cuadratic divergence in light-cone quantization, which is rather atypical for gauge theories [4]. The "mothor! of alternate denominators" has been suggested as a possible solution to this problem [5]. However, in Appendix 4A we show that this mothod must be nised with caution if one wants to recover the usual Feyman answer for general perturbative processes.

Whereas the problem of the one-loop quadratically divergent self-cnerg. ocrurs also in $A^{+}=0$ gauge, any gauge different from light-cone gange, such as Fegrnuan 
gauge, poses extra problems in light-cone quantization. To see this, we consider the "jellyfish graph" (Fig. 4.2) with $n(n \geq 0)$ external photons inserted into the loop. For any $n$ we find a quadratic divergence in this diagram [6]. Furthermore, oxtra logarithmic divergences occur, which can be seen by power comnting of the diagram in Fig. $4.3 \quad[7,8]$.

In the following we demonstrate that extra divergences in light-cone fick theories can be associated with certain noncovariant terms appearing in the light-cone formalism. As an example, we investigate the $n=0$ jellyfish graph $l_{n=0}$ (which is actually' just the one-loop self-energy) with momentum $p=\left(p^{+}, p^{-}, p_{\perp}\right)$. Wo leave the explicit. calculation to Appendix $4 \mathrm{~B}$ and quote the result obtained after mass renomalization (throughout the paper we use the notation $p^{ \pm}=p^{0} \pm p^{3}, \gamma^{ \pm}=\gamma^{0} \pm \gamma^{3}$ )

$$
I_{n=0}=(p-m) B+(p-m)^{2} \dot{U}\left(p^{2}\right)+\left(\frac{\gamma^{+}}{p^{+}}-\frac{\bar{u} \gamma^{+} u}{2 m \eta^{+}}\right)
$$

or

$$
\frac{1}{4} \operatorname{tr}\left(\gamma^{-} I_{n=0}\right)=p^{-}\left(B-2 m \Sigma\left(p^{2}\right)\right)+\frac{2}{p^{+}} C
$$

In the following we want to imply that the integral $\int d \lambda^{2} \rho\left(\lambda^{2}\right)=0$ is alway's taken, i.e., one Pauli...Villars subtraction is assumed. In the example of above we find

$$
C \frac{\gamma^{+}}{p^{+}}=\frac{e^{2}}{16 \pi^{3}} \frac{\gamma^{+}}{p^{+}} \int d^{2} k_{\perp} \log \frac{m^{2}+k_{1}^{2}}{\lambda^{2}+k_{\perp}^{2}}
$$

The quadratic divergence can be identified with the term $(?$ in $(4.1)$ and is therefore associated with the noncovariant structure in the self-energy.

We note that the occurrence of noncovariant terms of the form $\left(\mathrm{A}, \mathrm{t} / \mathrm{p}^{+}\right.$is $\mathrm{nol}$ restricted to the one-loop selfenergy [9]. In fact, all noncovariant termes we have encountered have had this structure (for a discussion of vacuum polarization contributions see (hapter 5). 


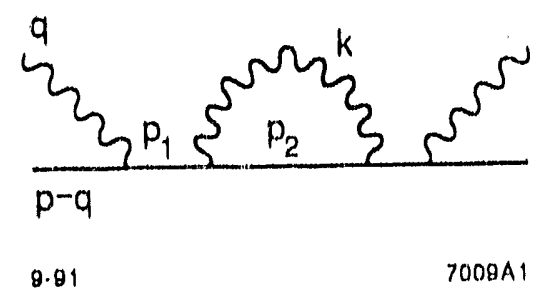

Figure 4.4. One-loop correction to Compton scattering.

As far as the self-energy is concerned, a method which is based on the correct tensor structure of the diagram can be proposed. This is possible since different. tensor components should be related by covariance:

$$
\operatorname{tr}\left(\gamma^{-} I_{s e l f-e n c r g y}\right)=\frac{p^{-}}{p^{+}} \operatorname{tr}\left(\gamma^{+} I_{s e l f-\operatorname{coc} g ! y}\right)
$$

where $p^{+}, p^{-}$correspond to the momentum of the fermion and $l_{\text {self }}$ - energy dentedes

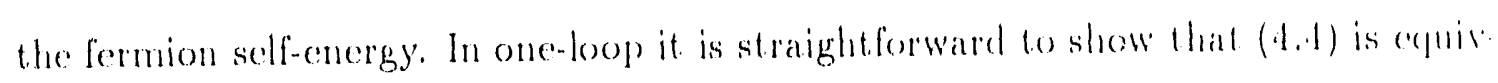
alent to the offective replacement.

$$
p_{2}^{-} \rightarrow p_{\text {lenergy-shell }} \frac{p_{i}^{-}}{p_{1}^{+}}
$$

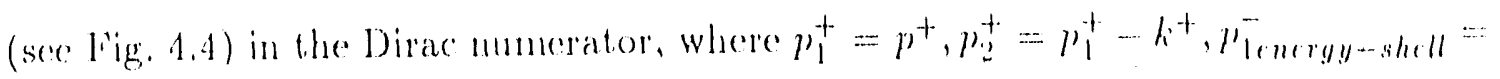
$p^{-}$. 'The momenta $\gamma^{+}, p^{-}$denote the total lighte-cone momentum and encerge respere tively. Here, $k^{t}$ is given by the light-cone momentum of the virtual photen. More generally, pe- pergy-ahell defines the light-cone energy one would obtain if lighte-come energy conservation was imposed. The replacement (4.5) experesses the "barl cont ponent." (i.e., $\left.\gamma^{+}\right)$in terms of the "good component" (i.e., $\gamma^{-}$) and thus remeler's the self-energy covariant by construction. Hence, the problem of the quardratie di. vergence is avoided in this case [10]. Fquation (4.4) can be gencratized to highere loop self-energy diagrams, provided all subloops are first rendered covariant and the noncovariant piece is of the form $\mathrm{C}^{+} / \mu^{+}$. 
Whereas the tensor method provides a useful practical tool for dealing with the quadratic divergence in selfenergy diagrams, the application of the tensor method for the cure of the jellyfish diagram with $n \geq 1$ is not straightlorward. 'Thiss is because' the different tensor components are not simply related in this case.

It should be noted that in $3+1$ dimensions the noncovasiant term in Eq. (4.2) and all other jellyfish diagrams can be eliminated more systematically, if the spectral conditions $[11,12]$

$$
\begin{array}{r}
\int d \lambda^{2} \mu\left(\lambda^{2}\right)=0 \\
\int d \lambda^{2} \lambda^{2} \mu\left(\lambda^{2}\right)=0 \\
\int d \lambda^{2} \lambda^{2} \log \left(\lambda^{2}\right) \rho\left(\lambda^{2}\right)=0
\end{array}
$$

are introduced which corresponds to the introduction of there Panli Villars ghost particles into the theory. However, this is awkward from a mumerical vieme, since llac mumber of degrees of freedom is enhanced dramatically in this casce. For example, a

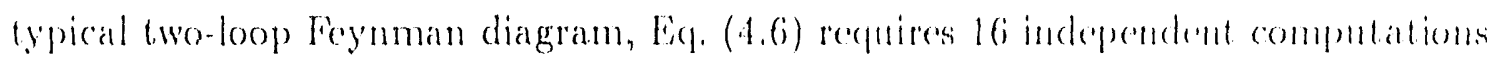
of its integrand at each integration point. 'This is in contrast to only at computations in a covariant approach. In addition, the quadratic divergences would be cancelled only among contributions from different. Pauli-Villars particles. However, for the purpose of numerical calculations, it is extremely inconvenient to cancen quadratic divergences among different diagrams, because of the limited acrurary of any mumer. icial procedure.

Hence, for practical purposes, it is necessary to develop a recipe which reduces the number of Pauli-Villars particles as well as subtracts guadratic divergencess localls: i.e., before integration. In this context we shall introduce the "mull-subtraction" as 
such a local procedure. For $n=0$ the idea of the null subtraction is based on the observation that the troublesome term in Eq. (4.2) is given by

$$
\frac{C}{p^{+}}=\frac{1}{8} \operatorname{tr}\left(\gamma^{-} I_{n=0}\right)_{p^{-}=0, p_{\perp}=0}
$$

where $C$ is independent of the external momenta. Hence, we define the null subtriation as a procedure where the "bad" component of a quadratically divergent graph or subgraph is subtracted for vanishing external (with respect to the divergent graph or subgraph) $p^{-}$and $p_{\perp}$ momenta, while keeping $p^{+} \geq 0$. In the above example we obtain for the null subtraction

$$
I_{N u \|}=\frac{e^{2}}{16 \pi^{3}} \int_{0}^{1} \frac{a^{\prime} x d^{2} k_{\perp}}{x(1-x)} \frac{\frac{1}{2} \gamma^{\mu} \frac{\gamma^{+}}{p^{+}} \frac{k_{\perp}^{2}+m^{2}}{(1-x)} \gamma_{\mu}}{\left(-\frac{m^{2}+k_{\perp}^{2}}{(1-x)}-\frac{\lambda^{2}+k_{\perp}^{2}}{x}\right)} .
$$

Performing replacements similar to those given in Appendix $4 \mathrm{~B}$ yiclds

$$
\begin{aligned}
I_{n u l l} & =-\frac{e^{2}}{16 \pi^{3}} \frac{\gamma^{+}}{p^{+}} \int_{0}^{1} d x \int d^{2} k_{\perp} \frac{\lambda^{2}-m^{2}}{k_{\perp}^{2}+\lambda^{2}\left(1-x^{\prime}\right)+m^{2} \cdot x^{2}} \\
& =\frac{e^{2}}{16 \pi^{3}} \frac{\gamma^{+}}{p^{+}} \int_{0}^{1} d x \int d^{2} k_{\perp} \frac{d}{d x} \log \left(m^{2} x+\lambda^{2}\left(1-x^{2}\right)+k_{\perp}^{2}\right) \\
& =\frac{c^{2}}{16 \pi^{3}} \frac{\gamma^{+}}{p^{+}} \int d^{2} k_{\perp} \log \frac{m^{2}+k_{\perp}^{2}}{\lambda^{2}+k_{\perp}^{2}} .
\end{aligned}
$$

What we encounter here is nothing but the noncovariant piece of Eq. (4.3). Hence, the null subtraction removes the quadratic divergence automatically in the correct waj.

Let us examine now the null-subtraction for the jellyfish graph for $n=1$ (which is actually the one-loop vertex correction in this case). 'The covariant answer is expected to be of the form [13]

$$
\Gamma^{\mu}=\gamma^{\mu} F_{1}^{i}\left(q^{2}\right)+\frac{i}{2 m} \sigma^{\mu \prime \prime} q_{\nu} F_{2}\left(q^{2}\right)
$$


Using the Gordon-decomposition, Eq. (4.10) can be rewritten as

$$
\begin{gathered}
\bar{u} \Gamma^{+} u=\bar{u} \gamma^{+} u\left(F_{1}\left(q^{2}\right)+F_{2}\left(q^{2}\right)\right)-\frac{1}{2}\left(p+p^{\prime}\right)^{+} F_{2}\left(q^{2}\right) \frac{\left(p+p^{\prime}\right)^{+}}{\sqrt{p^{+} p^{\prime}+}} \delta_{\lambda \lambda^{\prime}} \\
\bar{u} \Gamma^{-} u=\bar{u} \gamma^{-} u\left(F_{1}\left(q^{2}\right)+F_{2}\left(q^{2}\right)\right)-\frac{1}{2}\left(p+p^{\prime}\right)^{-} F_{2}\left(q^{2}\right) \frac{\left(p+p^{\prime}\right)^{+}}{\sqrt{p^{+} p^{\prime}+}} \delta_{\lambda \lambda^{\prime}},
\end{gathered}
$$

where $\lambda, \lambda^{\prime}$ denote the initial and final helicity respectively. The momenta $p$ and $p^{\prime}$ correspond to the initial and final fermion respectively. If one inserts the analytic form for the second term on the r.h.s. of Egs. (4.11) and (4.12), the sum Fil $\left(q^{2}\right)+$ $F_{2}\left(q^{2}\right)$ may be computed in two different ways: $F_{1}\left(q^{2}\right)+F_{2}\left(q^{2}\right)$ can be obtainer from the $\Gamma^{+}$current by means of Eq. (4.11). This is straightforward, since wo do not expect trouble in this case $[14,15]$. However, the extraction of $F_{1}\left(q^{2}\right)+F_{2}\left(q^{2}\right)$ by means of Eq. (4.12), i.e., by computing the $\mathrm{I}^{-}$current requires a null subtraction which takes the form

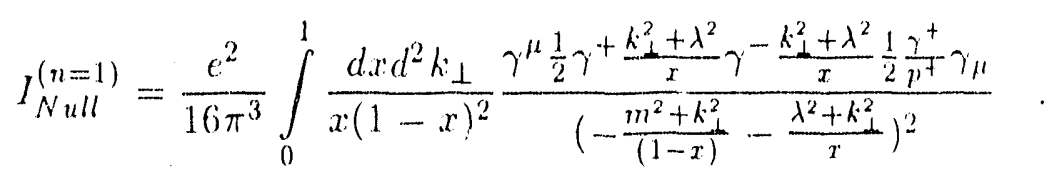

Note that we only subtract the $\gamma^{+}$component for zero external $p^{-}$and $p \perp$ momenta. If the null subtraction removes the quadratic divergences correctly, the result for $F_{1}\left(q^{2}\right)+F_{2}\left(q^{2}\right)$ should be the same in both cases. We have checked this statement numerically [16]. Hence, the null sutitraction restores the covariant answer also in the case of the $n=1$ jellyfish graph.

If we take those results, together with the fact that the one-loop Ward identities are fulfilled for the good components in LCPTh, one can say that the null subtraction preserves the Ward identities at one-loop (for external fermion lines on shell).

It should also be mentioned that we have checked the null subtraction methol for the case of the two-loop rainbow self-energy in Fig. 4.5. More interesting, how- 


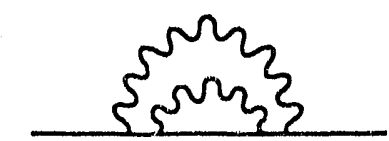

9.81

$7009 A 6$

Figure 4.5. Two-loop rainbow self-energy diagram.

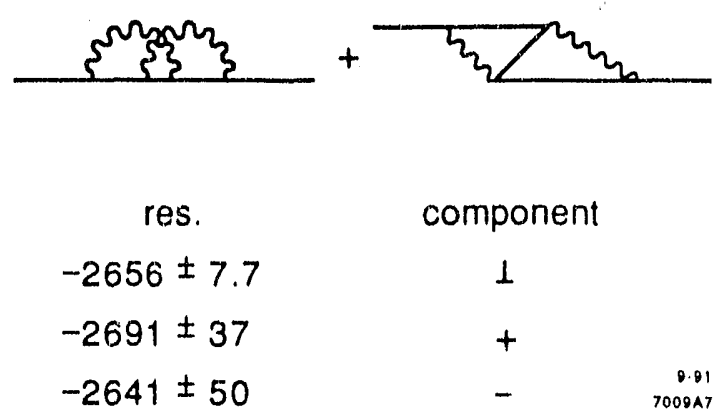

Figure 4.6. The two-loop self-energy contribution of the electron is expected to be of the form $A+B \not$, where $p$ corresponds to the external fermion momentum. 'The result res shows the extraction of $B$ by means of the various components of $p$.

ever, is the two-loop self-energy of Fig. 4.6 since it contains two $n=1$ jellyfish subdiagrams [17]. The corresponding null subtractions are

$$
\begin{aligned}
& I_{1}=\frac{e^{2}}{\left(16 \pi^{3}\right)^{2}} \int_{0}^{1} d^{2} k_{1 \perp} d x \int_{0}^{1-x} d^{2} k_{2 \perp} d y \frac{1}{x y(1-x)(1-y)(1-x-y)} \\
& \times \frac{\gamma^{\mu} \frac{1}{2} \gamma^{+} \frac{\lambda^{2}+k_{\lambda \perp}}{x} \gamma^{-} \frac{\lambda^{2}+k_{\perp}^{2}}{x} \frac{1}{2} \frac{\gamma^{+}}{p^{+}} \gamma_{\mu}\left(p_{2}+m\right) \gamma_{-}}{\left(-\frac{m^{2}+k_{1 \perp}^{2}}{(1-x)}-\frac{k_{1 \perp}^{2}+\lambda^{2}}{x}\right)\left(-\frac{m^{2}+k_{1 \perp}^{2}}{(1-x-y)}-\frac{k_{1 \perp}^{2}+\lambda^{2}}{x}-\frac{\lambda^{2}}{y}\right)\left(p^{-}-\frac{m^{2}+\left(p_{\perp}-k_{2 \perp}\right)^{2}}{(1-y)}-\frac{k_{2 \perp}^{2}+\lambda^{2}}{y}\right)}
\end{aligned}
$$

where $p_{2}=\left(1-y, p^{-}-\left(\lambda^{2}+k_{2 \perp}\right) / y, p_{\perp}-k_{2 \perp}\right)$ and 


$$
\begin{aligned}
& I_{2}=\frac{e^{2}}{\left(16 \pi^{3}\right)^{2}} \int_{0}^{1} d^{2} k_{1} \perp d x \int_{0}^{1-x} d^{2} k_{2} \perp d y \frac{1}{x y(1-x)(1-y)(1-x-y)}
\end{aligned}
$$

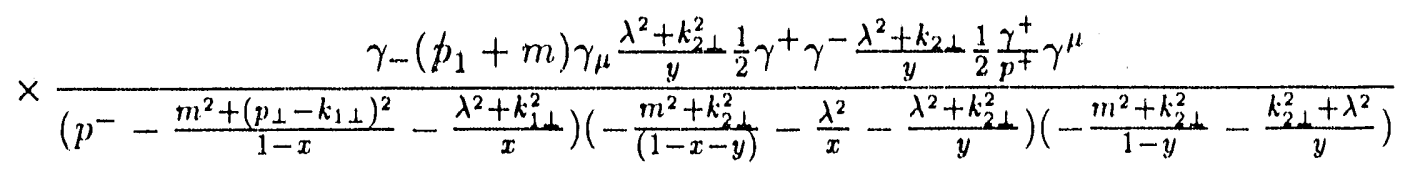

respectively. Figure 4.6 shows the result of the numerical integration for different. components. The result is that the null subtraction eliminates the quadratic divergence and restores a covariant form within the error of the calculation.

The general definition of the null-subtraction of the n-photon jellyfish graph

$$
\begin{aligned}
I_{J_{n}:=} & \frac{e^{2}}{\left(16 \pi^{3}\right)} \int_{0}^{p^{+}} d k^{+} d^{2} k_{\perp} \\
& \times \frac{\Theta\left(p^{+}-k^{+}\right) \Theta\left(p^{+}-k^{+}-q_{1}^{+}\right) \cdots \Theta\left(p^{+}-k^{+}-\cdots-q_{n}^{+}\right)}{k^{+}\left(p^{+}-k^{+}\right)\left(p^{+}-k^{+}-q_{1}^{+}\right) \cdots\left(p^{+}-k^{+}-q_{1}^{+}-\cdots-q_{n}^{+}\right)} \\
& \times \frac{\gamma^{\prime \prime}\left(p_{1}+m\right) \gamma^{\mu_{1}}\left(p_{2}+m\right) \gamma^{\mu_{2}} \cdots\left(p_{n}+m\right) \gamma_{\mu}}{\left(p^{-}-k^{-}-\frac{m^{2}+\left(p_{\perp}-k_{\perp}\right)^{2}}{p^{+}-k^{+}}\right) \cdots\left(p^{-}-k^{-}-\frac{m^{2}+\left(p_{\perp}-k_{\perp}-q_{1 \perp}-\cdots-q_{n \perp}\right)^{2}}{p^{+}-k^{+}-q_{1}^{+} \cdots-q_{n}^{+}}\right)}
\end{aligned}
$$

with external fermion momentum $p=\left(p^{+}, p^{-}, p_{\perp}\right)$ and momentum $q_{i}=\left(q_{i}^{+}, q_{i}^{-}, q_{i \perp}\right)$ for the $\mathrm{i}$-th external photon line is given by [18]

$$
\begin{aligned}
& I_{N u l l}^{n}=\frac{e^{2}}{\left(16 \pi^{3}\right)} \int_{0}^{p^{+}} d k^{+} d k_{\perp} \\
& \times \frac{\Theta\left(p^{+}-k^{+}\right) \Theta\left(p^{+}-k^{+}-q_{1}^{+}\right) \cdots \Theta\left(p^{+}-k^{+}-\cdots-q_{n}^{+}\right)}{k^{+}\left(p^{+}-k^{+}\right)\left(p^{+}-k^{+}-q_{1}^{+}\right) \cdots\left(p^{+}-k^{+}-q_{1}^{+}-\cdots-q_{n}^{+}\right)} \\
& \times \frac{\gamma^{\mu} \frac{1}{2} p_{1}^{-} \gamma^{+} \gamma^{\mu_{1}} \frac{1}{2} p_{2}^{-} \gamma^{+} \gamma^{\mu_{2}} \ldots \frac{1}{2} p_{n}^{-} \gamma^{+} \gamma_{\mu}}{\left(\frac{-k_{+}^{2}+\lambda^{2}}{k^{+}}-\frac{k_{\perp}^{2}+\lambda^{2}}{p^{+}-k^{+}}\right)\left(\frac{-k_{\perp}^{2}+\lambda^{2}}{k^{+}}-\frac{k_{\perp}^{2}+\lambda^{2}}{p^{+}-k^{+}-q_{1}^{+}}\right) \cdots\left(\frac{-k_{\perp}^{2}+\lambda^{2}}{k^{+}}-\frac{k_{\perp}^{2}+\lambda^{2}}{p^{+}-k^{+}-q_{1}^{+}-q_{2}^{+}-\cdots q_{n}^{+}}\right)}
\end{aligned}
$$


where $k^{-}=\left(k_{\perp}^{2}+\lambda^{2}\right) / k^{+}$. The fermion light-cone energies $p_{i}^{-}$are given by $p_{i}^{-}=$ $-\left(k_{\perp}^{2}+\lambda^{2}\right) / k^{+}$, if $p_{i}^{-}$is set on energy-shell i.e., the $i$-th fermion line does not extends over more than one intermediate state [5]. The on-mass-shell case yields $p_{i}^{-}=$ $\left(m^{2}+k_{\perp}^{2}\right) /\left(p^{+}-k_{1}^{+}-q_{1}^{+}-\cdots q_{i}^{+}\right)$. Note that the null subtraction in (4.17) is to be used in combination with only one Pauli-Villars particle. Thus, the number of degrees of freedom is considerably reduced. The was possible since all higher-loop) noncovariant terms are independent of the photon mass. We elaborate on this issuc in more detail in the next chapter.

The null subtraction was developed to deal consistently with quadratic divergences, in particular in the context of a numerical treatment. In Chapter 6 it is shown that the occurrence of noncovariant terms is due to nonvanishing surface and zero mode contributions in light-cone quantized field theories. An alternative methorl based on the addition of noncovariant counterterms to cure these problems will be proposed in the following chapters.

\subsection{Light-cone quantization in light-cone gauge}

For nonperturbative methods such as DLCQ or the light-front Tamm-Dancoff procedure, $A^{+}=0$ gauge is by far the most favorable choice among all ganges. This is due to the fact that ghosts and spurious degrees of freedom should not occur in this case. Furthermore, it seems to be the only gauge where canonical light-cont quantization is tractable, since it avoids having the $A^{+}$field in the denominator after solving the constraint equation for the left-handed spinors. In addition, only' light-cone quantization in $A^{+}=0$ gauge provides a convenient extraction of hadronic structure functions and, therefore, ensures an intuitive picture of high-energy scattering processes. Due to our discussion of the previous section, we may add the fact that quadratic divergences and noncovariant terms are restricted to a much smaller' set of diagrams, compared to any other gauge. However, as a noncovariant gange. 
$A^{+}=0$ requires a careful regularization of its $k^{+}$singularity, in particular because the covariant structure in a Hamilton formulation is already lost. Many procedures have been given in literature to regulate the light-cone gauge singularity [19-21]. In any event, every prescription gives rise to the introduction of a regularization parameter $\epsilon$ into the theory. It is essential for analytic, as well as numerical calculations, to ensure independence of physical quantities on the $\epsilon$ regulator.

In this section, we want to focus on $\epsilon$ prescriptions, which are easy enough to implement, i.e., they are of potential interest for practical applications in DCLQ or the light-front Tamm-Dancoff procedure. In addition, we investigate, in the particular example of the anomalous magnetic moment of the electron $(g-2) / 2$, the sensitivity of physical observables to a truncation at finite $\epsilon$. We start out with the light-cone gauge propagator, which has the form

$$
d_{\mu \nu}=-g_{\mu \nu}+\frac{\eta_{\mu} k_{\nu}+\eta_{\nu} k_{\mu}}{\eta \cdot k}
$$

where $\eta \cdot k:=k^{+}[23]$. One possibility to regulate the $\eta \cdot k$ singularity is given by

$$
d_{\mu \nu}=-g_{\mu \nu}+\frac{\eta_{\mu} k_{\nu}+\eta_{\nu} k_{\mu}}{\eta \cdot k} \Theta(\eta \cdot k-\epsilon)
$$

Note that the $\Theta$-function of the second term does not regulate the gauge piece only, but also all energy denominators which will multiply this term. Since gauge invariance in QED should occur locally (or quasi-locally [25]) we expect the correct result for the anomalous magnetic moment of the electron for any value for t between zero and one. This is exactly what we observe in our numerical calculations. It is instructive to see how the contributions of single diagrams add to the gange invariant answer. This is shown in Figs. 4.7 and 4.8. We remark that contributions of single diagrams grow logarithmically if e gets small, which makes it more difficult to maintain the numerical accuracy for small values of 6 . In order to obtain these 
graph

1.

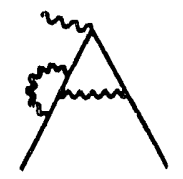

2.

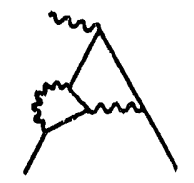

3.

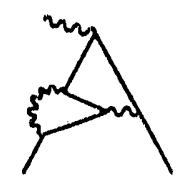

4.

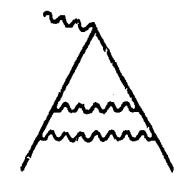

5.

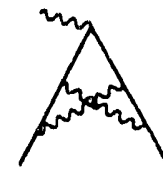

6.

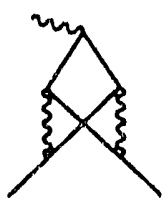

7.

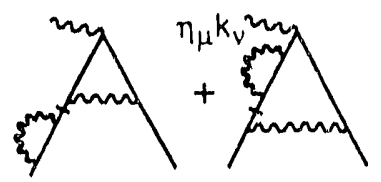

8.

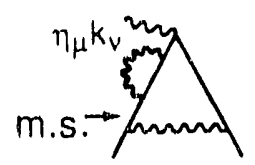

9.

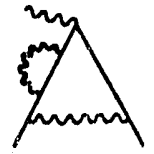

Total Result res

4941

$-172.9$

2.34

2.2

0.05

9

0.48

$-121.1$

$-3952$

2.0

$-428.8$

2.7

$-137.8$

5

$9 \cdot 91$

Figure 4.7. Contributions res of single LCPTh diagrams to the anomalums magnetic moment of the electron $a=(g-2) / 2$ to fourth order in light-cone gauge fur different values of the light-cone gauge cutoff $t[24]$. 


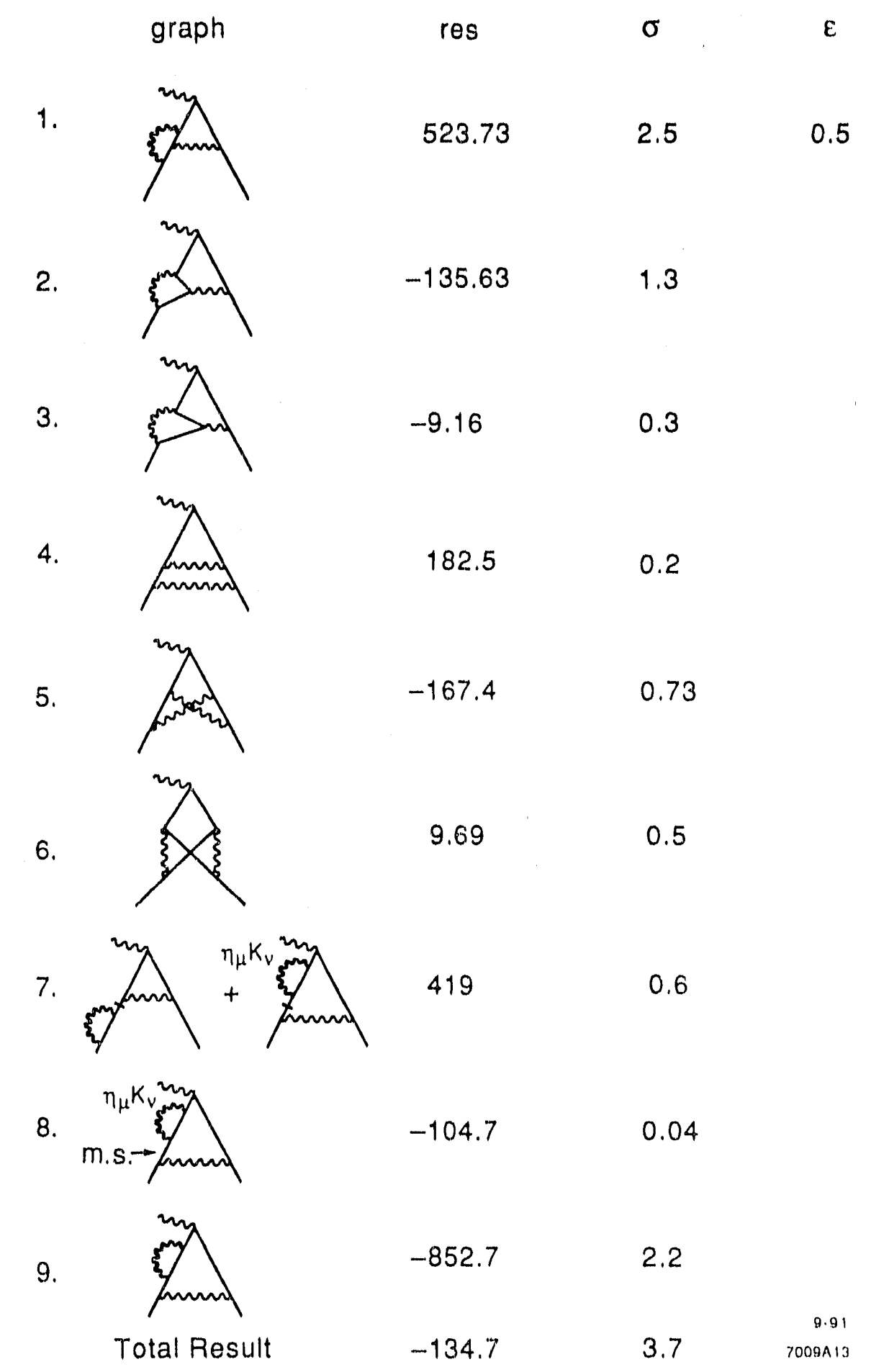

Figure 4.8. Fourth-order correction to the electron anomaly' in light-cone gauge for a different value of its gauge regulator. The analytic Feynman answer is given by -137.2 for $\alpha / 2 \pi=10$. 


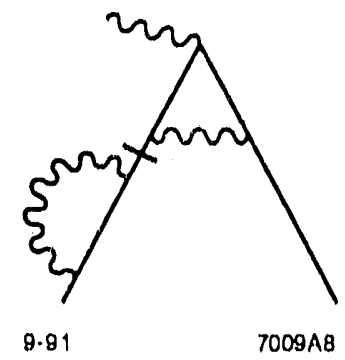

Figure 4.9. Instantaneous contribution to the external wavefunction correction in light-cone gauge.

results, it was essential to include the instantaneous self-energy diagram of Fig. 4.9 which vanishes in Feynman gauge. This is because the external solf-energy diagran. does contain a double pole in $A^{+}=0$.

The price we pay for the complete e-jndependence of physical observables for the regularization introduced in $\mathrm{Eq} .(4.19)$ is that for $\eta \cdot k<0$ the computation is carried out essentially in Feymman gauge. Indeed we find

$$
\eta_{\mu} d^{\mu \nu}(\epsilon)=-g^{+-}(1-\Theta(\eta \cdot k-c)) \neq 0
$$

for $\eta \cdot k<\epsilon$. Basically, any prescription which regulates the second term in licl. (1.16) different from the first one exhibits this feature. 'This is why', cven in light-cone ganger, the existence of ghosts cannot be excluded in general [26]. From a technical point of view Eq. (4.19) means that the jellyfish problem does occur even in $A^{+}=0$ gauge. The only exception to this case is given by a regularization, introduced through

$$
d_{\mu \nu}=\left(-g_{\mu \nu}+\frac{\eta_{\mu} k_{\nu}+\eta_{\nu} k_{\mu}}{\eta \cdot k}\right) \Theta(\eta \cdot k-1),
$$

which means that $A^{+}=0$ gauge is strictly obtained even at finite c, i.c., $A^{+}(1)=$ 0 . The advantage of this choice is the absence of ghosts and the jellyfish problem at finite $\epsilon$. However, regularization (4.21) will, in general, truncate also physical contributions to Feynman integrals. Thus, correct physical answers are recorered 
Table 4.1. Total answer for the electon anomaly to fourth order in light-cone gauge for different values of the gauge regulator. The analytic Feynman answer is given by $a=-131.4 \ldots(\alpha / \pi)^{2}$.

\begin{tabular}{|c|c|}
\hline$\delta \mathrm{a}$ & $\epsilon$ \\
\hline$-128.3 \pm 3.3$ & 0.0 \\
$-125.6 \pm 1.8$ & 0.01 \\
$-105.3 \pm 1.1$ & 0.05 \\
$57.5 \pm 0.1$ & 0.1 \\
\hline
\end{tabular}

only in the $\epsilon \rightarrow 0$ limit. For the purpose of practical applications, such as DLCQ, onc can investigate the numerical significance of such a truncation. In Table 4.1 we present the result for the computation of $(g-2) / 2$ for finite c, using the prescription in Eq. (4.21).

\subsection{Regulators in DLCQ}

Nonperturbative methods should generally be compatible with perturbation theory in the weak coupling domain of a theory. In lattice Q(CD), for example, the data scale like the one-loop $\beta$-function for weak coupling. This important feature indicates the recovery of the correct continuum field theory for small values of the lattice spacing. A Hamiltonian formulation of field theory, such as DI,CQ, should in principle reproduce correct perturbative results for any scattering process to finite order in the coupling. Thus, the calculation of $g-2$ to fourth order provides a powerful consistency check as well as an ideal testing ground for those methods.

We start our discussion with the test of the global cut-off, which is commonly used in DLCQ [22]. The global cut-off regulates an intermediate state with n particles according to

$$
\Theta\left(-\sum_{i=1}^{n} \frac{m_{i}^{2}+k_{i \perp}^{2}}{x_{i}}+\Lambda^{2}\right)
$$




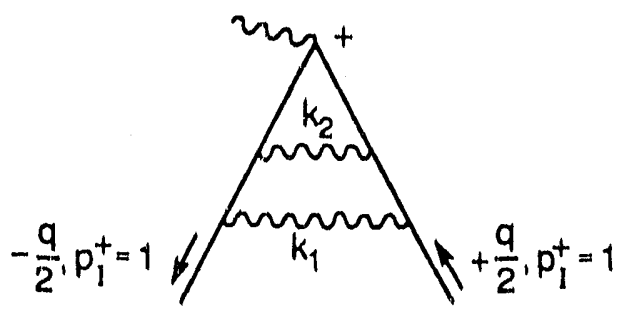

$7009 A 10$

Figure 4.13. Ladder diagram contribution to the electron anomaly in fourth order. where $x_{i}, k_{i \perp}, m_{i}$ refers to the light-cone $x$, the perp.momenta and the mass of the i-th particle respectively. $\Lambda$ denotes the ultraviolet cut-off, which is taken to infinity at the end of the calculation. Our result for the calculation of graph $1+2$ in Fig. 4.1 is $R_{1+2}=(-0.34 \pm 0.005) \alpha^{2} / \pi^{2}$ which is to be compared with the analytic result by Petermann: $R_{P}=-0.3285 \ldots \alpha^{2} / \pi^{2}$. The result for the ladder graph using the global cut-off is $(0.658 \pm 0.006) \alpha^{2} / \pi^{2}$. However, the correct answer is given by $R=0.778 \alpha^{2} / \pi^{2}$. Thus, the global cut-off does not recover the correct continumm answer in the limit $\Lambda \rightarrow \infty$. In order to understand what has happened, we recall the theta-function in the $\vec{q} \rightarrow 0$ lirnit for the counterterm (sce graph 4 in Fig. 4.1)

$$
\Theta\left(-\frac{m^{2}+k_{2 \perp}^{2}}{1-x}-\frac{\lambda^{2}+k_{2 \perp}^{2}}{x}+\Lambda^{2}\right)
$$

where $m, \lambda$ denote the fermion mass and the photon mass, respectively. Here, the variables $k_{2 \perp}$ and $x$ correspond to the loop momentum of the virtual photon [27]. However, the theta-function of the second intermediate state of the diagram corresponding to Fig. 4.13 is given by

$$
\Theta\left(-\frac{m^{2}+\left(k_{2 \perp}+k_{1 \perp}\right)^{2}}{1-x-y}-\frac{\lambda^{2}+k_{2 \perp}^{2}}{y}-\frac{\lambda^{2}+k_{1 \perp}^{2}}{x}+\Lambda^{2}\right)
$$

Obviously, (4.24) does not reduce to (4.23) in the large $k_{2 \perp}$ limit and hence does not allow a factorization of its infinite contribution. This effect induces the observed deviation from the correct answer in the $\Lambda \rightarrow \infty$ limit. 


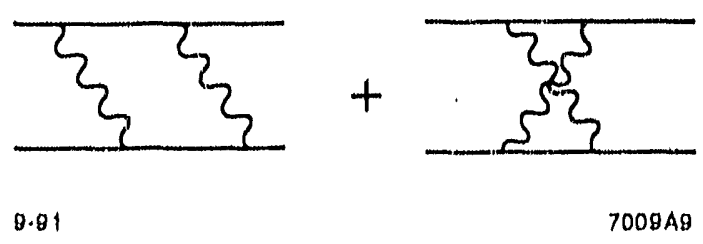

Figure 4.10. Correction to $e^{-t} c^{-}$scaltering.

Recently, the so-called local cut-ofl has been proposed [28], which restricts the difference in the invariant mass locally, i.e, at a given vertex only, to values less than $\Lambda^{2} / x$. Here $x$ is given by the fraction of the light-cone momentum which flow's through the vertex under consideration. Hence, (4.24) gets replaced by

$$
\left|-\frac{m^{2}+\left(k_{1 \perp}+k_{2 \perp}\right)^{2}}{1-x-y}-\frac{\lambda^{2}+k_{21}^{2}}{y}+\frac{m^{2}+k_{1 \perp}^{2}}{1-x}\right| \leq \frac{\Lambda^{2}}{1-x} .
$$

Changing variables according to $y=(1-x) \hat{y}, k_{2 \perp}=\dot{k}_{2 \perp}-\dot{y} k_{1 \perp}$ and $\dot{\Lambda}^{2}=\Lambda^{2}-m^{2}$ reduces (4.25) to (4.23) and, thus, avoids the problem of the global culloff, Indered our calculations show that the local cut-of reproduces the correet result for the ladelen graph. Unfortunately, it leads to the incorrect answer for graph 1+2 in ligg. 4.1. It is straightforward to show that the local cut-ofl violates gange invariance allendy at the tree-level $[30]$.

Other theta-function cutoffs, which have been proposed [31], are also doomed to failure, unless a noncovariant countertem is involed. The reason is that they. depend on momenta, i.e., derivatives only. However, a gange invariant regulator would require a functional dependence on covariant derivatives instead.

In Appendix $4 \mathrm{C}$ we demonstrate the implementation of dimensional regulariattion on the light-cone, 


\subsection{Summary}

We have shown that light-cone quantization in Foymman gange leadis lo inn infinite number of quadratically divergent LCP'lli diagrams at the onc-loop level. 'I'le' problem occurs for selfenergy diagrams where n-photons $(n \geq 0)$ are inserted inles the loop ("n-photon jellyfish problem"). We constructed a local representation of noncovariant counterterms, called the "null-subtraction," in order lo remove those divergences from the formalism.

In principle, also light-cone quantization in light-cone gange exhibits this finture for all $n$ (and not only for $n=0$ ). This is due to the fact that most regularizations of the light-cone gange sing alarity reduce the small ar behavion of the photom prepagator to that in Feymman gange. In this case, the null subteration con he used in the sinne way.

In Section 2 we evaluated the fonth-order correction to the anomalons magnetio moment of the electron in light-cone gauge and reproduced the analytic lieg'uman gauge result by Petermann. It was shown that a finite truncation of the $h^{+} \simeq 0$ region can lead to a significant modification of the contimmmu result.

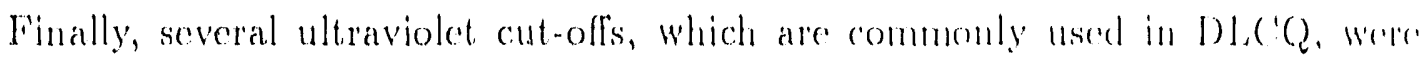
tested in porturbation theory. It was shown that those regulators do not recosen Ilue correct continum field theory in the $\Lambda \rightarrow \infty$ limit.

Appendix $1 \mathrm{C}$ demonstrates the introduction of dimensional regularization inte the light-cone formalism. 


\subsection{Appendix 4A}

In this section we discuss the "mothod of a" arnate denominators" which was introduced in Rof. (5) as a possible way of removing quadratic divergences in the lighte. cone formulation, for illustration the one- loop correction to the (iomptom graph, shown in Fig. 4.4, yiolds [32]

$$
\begin{aligned}
& I_{C o m p}=\frac{\pi k\left(p_{1}+m\right)}{p_{1}^{+}\left(p_{i}^{-}-\cdots \cdot \frac{m^{2}+p_{12}^{2}}{p_{1}^{+}}\right)}
\end{aligned}
$$

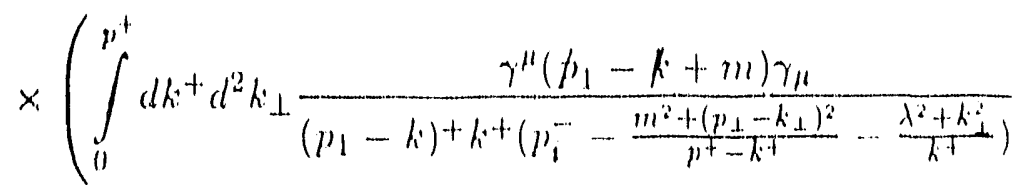

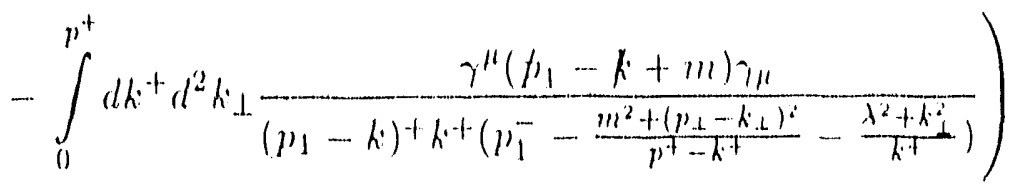

$$
\begin{aligned}
& \times \frac{\left(p_{1}+m\right) k^{*} u}{p_{1}^{+}\left(\mu_{i}^{-}-\frac{m^{2}+p_{-1}^{2}}{p_{1}^{+}}\right)}
\end{aligned}
$$

I'le second term is the alternate denominator (a.d.) subtraction which is designest to cancel the quadratic divergence in the first term as woll as perform the mass renormalization (see Fig. 4.11). The and term is obtamed by replacing the intial energy $\mu_{i}^{--}$in the energy-denominator of the quadratically divergent subgraph by its adjacent energy $p_{1}^{--}$which is, in case of the selfeenergy diagram in lïg. 4.4, apual to the mass shell energy $i_{1}^{-}$(sec below).

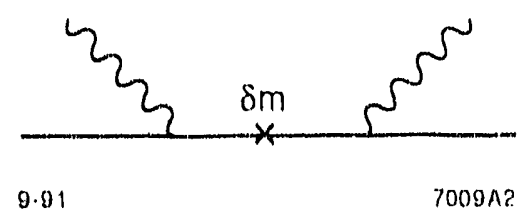

Figure 4.11. Mass correction to chectron (ompton scattering. 
Obviously, the quadratic divergence is subtracted in this procedure since it is independent of the incoming energy. However, it remains to be shown that the mass subtraction of Fig. 4.11 is carried out correctly, using the a.d. method. Note that the a.d. term $I_{a . d}$. of Eq. (4.26) is obtained by performing the $k^{-}$integration of

$$
\begin{aligned}
I_{a . d .}= & \int d^{4} k \frac{\bar{u} k\left(p_{1}+m\right)}{\left(p_{1}^{2}-m^{2}+i \epsilon\right)} \\
& \times\left(\frac{\left.\gamma_{\mu}\left(p_{1}-k\right)+m\right) \gamma^{\mu}}{\left(\left(\tilde{p}_{1}-k\right)^{2}-m^{2}+i \epsilon\right)^{2}\left(k^{2}-\lambda^{2}+i \epsilon\right)}\right) \frac{\left(\phi_{1}+m\right) k^{*} u}{\left(p_{1}^{2}-m^{2}+i \epsilon\right)}
\end{aligned}
$$

Here, $\tilde{p}_{1}$ is on-shell, i.e., $\tilde{p}_{1}^{\mu}=p_{1}^{\mu}$ for $\mu \neq-$ and $\tilde{p}_{1}^{-}=\left(m^{2}+p_{1}^{2}\right) / p_{1}^{+}$. Howerer. Hhe usual Feynman counterterm is given by

$$
\begin{aligned}
I_{\delta m}= & \frac{1}{2 m} \int d^{4} k \frac{\bar{u} k\left(p_{1}+m\right)}{\left(p_{1}^{2}-m^{2}+i c\right)} \\
& \times\left(\frac{\left.\bar{u}\left(\tilde{p}_{1}\right) \gamma_{\mu}\left(p_{1}-k\right)+m\right) \gamma^{\mu} u\left(\tilde{p}_{1}\right)}{\left(\left(\tilde{p}_{1}-k\right)^{2}-m^{2}+i c\right)^{2}\left(k^{2}-\lambda^{2}+i c\right)}\right) \frac{\left(p_{1}+m\right) k^{*} u}{\left(p_{1}^{2}-m^{2}+i c\right)}
\end{aligned}
$$

Obviously, there is a difference between these two expressions lecaluse of the spimes $u\left(\tilde{p}_{1}\right)$ and $\bar{u}\left(\dot{p}_{1}\right)$ which project out the $\delta m$ piece from the selfenergy in Eq. (1.2s). Thus, we conclude that the ad. method must be used with caution. Howerer. if une. ignores the double instantaneous gaph of Fig. 4.12 for a monent, at least me of He. fermions is on-shell and the corresponding propagator

$$
\frac{p_{1}+m}{p_{1}^{2}-m^{2}+i}
$$

gets replaced by

$$
\frac{p_{1}+m}{p_{1}^{2}-m^{2}+i c}=\sum_{s} \frac{u\left(j_{1}, w\right) \pi\left(j_{1} \ldots\right)}{p_{1}^{2}-m^{2}+u} .
$$

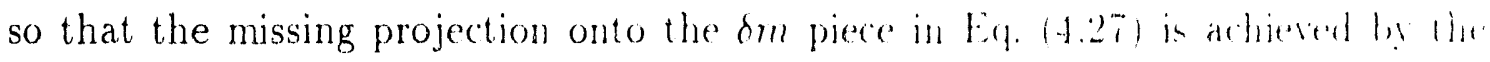

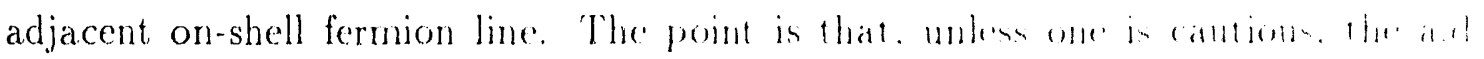




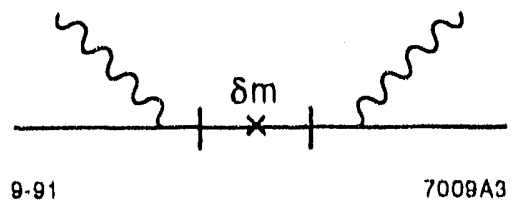

Figure 4.12. Double instantancous diagram to electon Compton scattering.

method treats the double instantaneous graph incorrectly by subtracting a nonzero contribution [33].

Thus, if one modifies the a.d. method such that the subtraction is excluded from the double instantaneous self-energy diagran, the usual (Feynman-) answer can be obtained [34]. 


\subsection{Appendix 4B}

In this section we prove that the $n=0$ jellyfish graph (which is actually just the one-loop self-energy) with momentum $p=\left(p^{+}, p^{-}, p_{\perp}\right)$ has the form

$$
I_{n=0}=(p-m) B+(\not p-m)^{2} \Sigma\left(p^{2}\right)+\left(\frac{\gamma^{+}}{p^{+}}-\frac{\bar{u} \gamma^{+} u}{2 m p^{+}}\right) C
$$

after mass renormalization. In the following we want to imply that the integral $\int d \lambda^{2} \rho\left(\lambda^{2}\right)=0$ is always taken, i.e., one Pauli-Villars subtraction is assumed. LCPTh yields for the $\gamma^{-}$and $\gamma_{\perp}$ component for the $n=0$ jellyfish graph

$$
\begin{aligned}
\Sigma^{-, \perp}= & -\frac{e^{2}}{16 \pi^{3}} \int_{0}^{1} d x d^{2} k_{\perp} . \\
& \times \frac{\gamma^{\mu}(\underline{p}-\underline{k}) \gamma_{\mu}}{\left.\left(k_{\perp}-p_{\perp} x\right)^{2}-p_{\perp}^{2} x^{2}-p^{-} p^{+} x(1-x)+\left(m^{2}+p_{\perp}^{2}\right) x^{2}+\lambda^{2}(1-x)\right)}
\end{aligned}
$$

where the "good" vectors $\underline{p}=\left(p^{+}, 0, p_{\perp}\right), \underline{k}=\left(k^{+}, 0, k_{\perp}\right)$ have been introduced. "The quantity $x$ is given by the relative momentum carried by the virtual photon, i.e., $x=$ $k^{+} / p^{+}$. Rewriting the denominator in terms of the four momentum $p^{2} \equiv p^{+} p^{-}-p^{2}$. and shifting integration variables yields

$$
\Sigma^{-, \perp}=-\frac{e^{2}}{8 \pi^{3}} \int_{0}^{1} d x d^{2} k_{\perp} \frac{p(1-x)}{-k_{\perp}^{2}+x(1-x) p^{2}-m^{2} x-\lambda^{2}(1-x)}
$$

For the $\gamma^{+}$component we find

$$
\Sigma^{+}=-\frac{e^{2}}{8 \pi^{3}} \frac{1}{2} \frac{\gamma^{+}}{p^{+}} \int_{0}^{1} d x d^{2} k_{\perp} \frac{\frac{m^{2}+k_{\perp}^{2}}{1-x}+p_{\perp}^{2}\left(1-l^{2}\right)}{-k_{\perp}^{2}+x(1-x) p^{2}-m^{2} l^{2}-\lambda^{2}(1-x)} .
$$

Since we are using Pauli-Villars regulator, the replacement $k_{-} \rightarrow p^{2} x(1-x)-m^{2} x-$ $\lambda^{2}(1-x)$ does not changa the value of the integral [35]. 'Thus,

$$
\mathrm{S}^{+}=-\frac{e^{2}}{8 \pi^{3}} \frac{1}{2} \frac{\gamma^{+}}{p^{+}} \int_{0}^{1} d x d^{2} k_{\perp} \frac{x p^{2}+m^{2}-\lambda^{2}+p_{1}^{2}\left(1-x^{2}\right)}{-k_{1}^{2}+x(1-x) p^{2}-m^{2} x^{2}-\lambda^{2}\left(1-x^{2}\right)}
$$


Using

$$
\begin{aligned}
x p^{2}+\left(m^{2}-\lambda^{2}\right) & =-\left[(1-2 x) p^{2}-m^{2}+\lambda^{2}\right]+(1-x) p^{2} \\
& =-\frac{d}{d x}\left[p^{2} x(1-x)-x m^{2}-(1-x) \lambda^{2}-k_{\perp}^{2}\right]+(1-x) p^{2}
\end{aligned}
$$

we obtain,

$$
\begin{aligned}
\Sigma^{+}= & \frac{e^{2}}{8 \pi^{3}} \frac{1}{2} \frac{\gamma^{+}}{p^{+}} \int_{0}^{1} d^{2} k_{\perp} d x \frac{d}{d x} \log \left(p^{2} x(1-x)-x m^{2}-(1-x) \lambda^{2}-k_{\perp}^{2}\right) \\
& -\frac{\epsilon^{2}}{8 \pi^{3}} \frac{1}{2} \gamma^{+} \int_{0}^{1} d^{2} k_{\perp} d x-\frac{(1-x) p^{-}}{-k_{\perp}^{2}+x(1-x) p^{2}-m^{2} x+\lambda^{2}(1-x)}
\end{aligned}
$$

Obviously, the last integral corresponds to the integral in $\mathrm{Eq} .(4.31)$ and is therefore part of the covariant answer.

However, the first integrand in Eq. (4.34) is noncovariant and leads to

$$
C \frac{\gamma^{+}}{p^{+}}=\frac{e^{2}}{16 \pi^{3}} \frac{\gamma^{+}}{p^{+}} \int d^{2} k_{\perp} \log \frac{m^{2}+k_{\perp}^{2}}{\lambda^{2}+k_{\perp}^{2}}
$$

'The total answer becomes

$$
\begin{aligned}
\delta m+I_{n=0}= & \frac{e^{2}}{16 \pi^{3}} \frac{\gamma^{+}}{p^{+}} \int_{0}^{1} d^{2} k_{\perp} d x \frac{d}{d x} \log \left(p^{2} x(1-x)-x m^{2}-(1-x) \lambda^{2}-k_{\perp}^{2}\right) \\
& -\frac{e^{2}}{8 \pi^{3}} \int_{0}^{1} d^{2} k_{\perp} d x \frac{(1-x)(p-m)}{-k_{\perp}^{2}+x(1-x) p^{2}-m^{2} x-\lambda^{2}(1-x)} \\
& +\frac{-(1+x) m}{-k_{\perp}^{2}+x(1-x) p^{2}-m^{2} x-\lambda^{2}(1-x)}
\end{aligned}
$$


where $\delta m$ denotes the mass correction. Performing mass renormalization yields

$$
\begin{aligned}
I_{n=0}= & \frac{e^{2}}{16 \pi^{3}}\left(\frac{\gamma^{+}}{p^{+}}-\frac{1}{2 m} \bar{u} \frac{\gamma^{+}}{p^{+}} u\right) \\
& \times \int_{0}^{1} d^{2} k_{\perp} d x \frac{d}{d x} \log \left(p^{2} x(1-x)-x m^{2}-(1-x) \lambda^{2}-k_{\perp}^{2}\right) \\
& -\frac{e^{2}}{8 \pi^{3}} \int_{0}^{1} d^{2} k_{\perp} d x \frac{(1-x)(p-m)}{-k_{\perp}^{2}+x(1-x) p^{2}-m^{2} x-\lambda^{2}(1-x)} \\
& -\frac{e^{2}}{8 \pi^{3}} \int_{0}^{1} d^{2} k_{\perp} d x \frac{1}{\left(-k_{\perp}^{2}+x(1-x) p^{2}-m^{2} x-\lambda^{2}(1-x)\right)} \\
& \times \frac{\left(1-x^{2}\right) x m\left(p^{2}-m^{2}\right)}{\left(-k_{\perp}^{2}+x(1-x) m^{2}-m^{2} x-\lambda^{2}(1-x)\right)} \cdot
\end{aligned}
$$

Thus, we obtain the form of the self-energy in Eq. (4.29). 


\subsection{Appendix 4C}

In this section, we demonstrate the use of dimensional regularization in light-cone quantization. For illustration we discuss the computation of the ladder diagram in Fig. 4.13. LCPTh yields

$$
\begin{aligned}
F_{L}= & \frac{e^{4}}{\left(16 \pi^{3}\right)^{2}} \int_{0}^{1} d x \quad d^{2} k_{1} \int_{0}^{1-x} d y d^{2(1-c)} k_{2} \frac{1}{x y(1-x)^{2}(1-x-y)^{2}} \\
& \times \frac{N\left(q, k_{1}, k_{2}\right)}{\left(m^{2}-\frac{m^{2}+k_{1}^{2}}{1-x}-\frac{k_{1}^{2}+\lambda^{2}}{x}\right)\left(m^{2}-\frac{m^{2}+\left(k_{1}+k_{2}\right)^{2}}{1-x-y}-\frac{k_{1}^{2}+\lambda^{2}}{x}-\frac{k_{2}^{2}+\lambda^{2}}{y}\right)}
\end{aligned}
$$

where $m, \lambda$ denote the fermion and photon mass respectively. The Dirac numerator is abbreviated by $N\left(q, k_{1}, k_{2}\right)$ and will be specified later. Note, that the $q$ dependence in the denominator can be dropped, in this particular example, since it gives nu contribution to the anomaly. Notice further that only the inner loop is ultraviolet divergent and requires regularization. The introduction of dimensional regularization according to

$$
\int d x \quad d^{2} k \rightarrow \int d x d^{2(1-1)}
$$

seems dangerous, in particular if the integrals are not absolutely convergent. Howcrer, (4.38) is a direct consequence of the definition of dimensional regularization [36] We have not yet encountered an example where (4.38) leads to additional difficulties (in comparison to one Pauli-Villars photon and fermion for example) in the light-cone formulation.

Shifting momenta and setting $m=1$ yields

$$
\begin{aligned}
F_{L}= & \frac{c^{4}}{\left(16 \pi^{3}\right)^{2}} \int_{0}^{1} d x d^{2} k_{1} \int_{0}^{1} d y \frac{y}{(1-x)^{4} x\left(1-\frac{1+k_{1}^{2}}{1-x}-k_{1}^{-}\right)^{2}} \\
& \times \int d^{2(1-c)} k_{2} \frac{N\left(q, k_{1}, k_{2}-\frac{y}{(1-x)^{2}} k_{1}\right)}{\left(\tilde{k}_{2}^{2}-\frac{y^{2}}{(1-x)^{2}} k_{1}^{2}+\frac{y(1-x-y)}{1-x}\left(-1+\frac{1+k_{1}^{2}}{1-x-y}+k_{1}^{-}+\frac{\lambda^{2}}{y}\right)\right)^{2}}
\end{aligned}
$$


where $\hat{k}_{2}=k_{2}+y /(1-x) k_{1}$ and $k_{1}^{-}=\left(k_{1}^{2}+\lambda^{2}\right) / x, k_{2}^{-}=\left(k_{2}^{2}+\lambda^{2}\right) / y$. If we exprand the numerator according to

$$
N\left(q, k_{1}, \tilde{k}_{2}-\frac{y}{1-x} k_{1}\right)=A \tilde{k}_{2}^{2}+B k_{1}+C
$$

the last integral can be performed analytically by means of

$$
\int \frac{d^{2 \omega} l}{(2 \pi)^{2 \omega}} \frac{l_{\mu} l_{\nu}}{\left(l^{2}+M^{2}\right)^{A}}-\frac{1}{(4 \pi)^{\omega} \Gamma(A)} \frac{1}{2} \delta_{\mu \nu} \frac{\Gamma(A-1-\omega)}{\left(M^{2}\right)^{A-1-\omega}}
$$

With the definition

$$
\begin{aligned}
f\left(k_{1}, x, y\right)= & \frac{y}{(1-x)^{4} x} \\
& \times \frac{1}{\left(1-\frac{1+k_{1}^{2}}{1-x}-k_{1}^{-}\right)^{2}} \frac{1}{\frac{-y^{2}}{(1-x)^{2}} k_{1}^{2}+\frac{y(1-x-y)}{1-x}\left(-1+\frac{1+k_{1}^{2}}{1-x-y}+k_{1}^{-}+\frac{x^{2}}{y}\right)}
\end{aligned}
$$

one obtains

$F_{L}=\frac{e^{4}}{\left(16 \pi^{3}\right)^{2}} \int_{0}^{1} d^{2} k_{1} \int_{0}^{1} d y f\left(k_{1}, x, y\right)\left(\left(A+c A^{\prime}\right) \pi(1-c)\left(\frac{1}{c}-\left(^{\prime} c u l\right)+\pi\left(\left|3 k_{1}\right|+c^{\prime}\right)\right)\right.$.

where we have written $A(c)=A+\epsilon A^{\prime} . A, A^{\prime}, B$ can be computed, using a algebraic manipulation program like REDUCE. (4.41) can be integrated mumerically. (', ul, is the Euler constant and given by $C_{c u l}=0.577 \ldots$.

The counterterm to Fig. 4.13 (see diagram 4 in Fig. 4.1) is computed in a similal way. It should be stressed that the pole in the one-loop vertex correction of diagram t in Fig. 4.1 not only cancels the pole in Eq. (4.41), but also gives rise to a finite contribution [37].

We have redone the entire fourth-order calculation using dincnsional regularizition. Unlike the computation of the ladder graph, in general one has to combine energy denominators first, before the analytic part of the integration can be carried 
out. In contrast to a covariant theory, only one additional a parameter is necessary in light-cone quantization. This is due to the fact that the photon propagator $1 /\left(k^{2}+i \epsilon\right)$ simply becomes $1 / k^{+}$in this case.

On the other hand, the coefficients $A, A^{\prime}, B$ are harder to extract in light-cone quantization since the fermion energies generally depend implicitly on the loop momentia.

An understanding of dimensional regularization is essential, if one wants to extend LCPTh to non-Abelian gauge theories. 


\section{REFERENCES for CHAPTER 4}

[1] G. P. Lepage and S. J. Brodsky, Phys. Rev, D22, 2157 (1980).

[2] A. Petermann, Helv. Phys. Acta 30, 407 (1957).

[3] C. M. Sommerfield, Ann. Phys. (NY) 5, 26 (1958).

[4] This is true with the exception of scalar electrodynamics which also contains a quadratic divergence of the one-loop self-energy.

[5] S. J. Brodsky, R. Roskies and R. Suaya, Phys. Rev. D8, 4574 (1973).

[6] This refers to the case where one picks always the $p_{i}^{-} \gamma^{+}$component in the Dirac numerator for the fermion lines. In order to make the trace nonzero, all external photon ines must provide a $\gamma^{-v e r t e x ~ t o ~ t h e ~ n u m e r a t o r . ~ T h u s, ~ t h e ~}$ problem does not occur in $A^{+}=0$ gauge for $n \geq 1$.

[7] In Feynman gauge those divergences cancel if all tince-orderings are summed. In the case of light-cone gauge, those divergences cancel identically due to properties of the Dirac trace.

[8] In the particular example of $(g-2)$ to fourth order, the jellyfish problem for $n \geq 1$ does not occur if the spin flip amplitude of the $I^{+}$current is used for the extraction of the anomaly. This is due to the fact that $\gamma^{+}$matrices cammot flip helicity.

[9] For an explicit two-loop example see Appendix 4(' of (hapter (6.

[10] The tensor method can be used for the one-loop self-energy in $A^{+}=0$ gange as well, as long as the replacement (4.5) is restricted to the gyn piece in the propagator only.

[11] C. Bouchiat, P. Fayet and N. Sourlas, Lett. al Nuovo (imeto, 9 (1972); S...). Chang and T.-M. Yan, Phys. Rev. D7, 1147 (1973). 
[12] Unfortunately, we are not able to prove this rigorously, However, we have checked the most relevant two-loop diagrams to fourth order and verified the statement in these cases.

(13) Provided the external fermion lines are on shell.

[14] In this case the null subtraction would subtract zero.

[15] Problems should not occur in this case since fermion light-cone energies do not contribute to the Dirac numerator because of $\gamma^{+} \gamma^{+}=0$.

[16] As an example we cite the extraction of $F_{1}+F_{2}$ for the choice of the initial (in) and final $(f)$ momentum given by $p_{i n}=(0.4,4.3,0.85,0)$ and $\eta_{f}=(1,1,0,0)$. In the case of the good current we obtain $F_{1}+F_{2}=-250.1 \pm 1.3$. 'This is to be compared with $F_{1}+F_{2}=-248.5 \pm 1.5$. In this calculation we used a Pauli-Villars cut-off $\Lambda=10 \mathrm{~m}$ and a photon mass $\lambda=10^{-4} \mathrm{~m}$, where $\mathrm{m}$ denotes the electron mass. For numerical reasons we chose $a / 2 \pi=10$.

[17] It should be noted that no overlapping quadratic divergences occur in this example. This is because $\gamma^{-}$insertions are necessary at 2 and 3 in order 10 make both jellyfish diagrams quadratically divergent. However, thus cuforces $\gamma^{+}$components at 1 and 4 (in Feynman gauge) which loads to a zoro result because only $\gamma^{+}$components contribute to the fermion lines in this casc. 'lhis result is consistent with the null subtraction.

[18] The case considered here involves incoming external photons only. If some of the photons are outgoing, the corresponding light-cone momentat $q_{i}^{+}$must be set negative in $(4.16)$.

[19] G. Leibbrandt, Rev. Mod. Phys. 59, 1067 (1987).

[20] A. Basseto, R. Soldati, Phys. Rev. D41, 3277 (1990).

[21] O. Piguet, G. Pollak, M. Schweda, Nucl. Phys, B328, 527 (1984).

[22] A. Tang, Ph.D. thesis, SLAC-REPORT-0351 (199()). 
[23] Note that the piece $\sim\left(\eta_{\mu} \eta_{\nu}\right) /(\eta \cdot k)^{2}$ is cancelled in phy'sical S'matrix elements by means of the instantaneous photon interaction, Effredively, instantalueons diagrams can be taken into account by putting those photon lines on energ. shell, which do not extend over more than one intermediate stater, In ordere to avoid severe infrared problems, nomperturbalive mothods must include instantaneous contributions if, and only if, the corresponding photon in flight contributes to the approximation [22].

[24] An ultraviolet cut-off $\Lambda=1000 \mathrm{~m}$ was used, where $m$ denotes the clectron mass. The residual $\Lambda$ dependence is within the error $(\sigma)$ of the calculation. For conveniance $a / 2 \pi=10$ was chosen, m.s. associated with a line indicatest

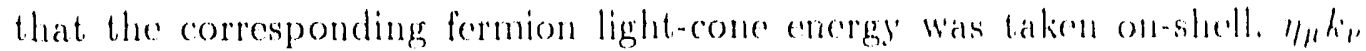
means that only the gange piece of the corresponding photon propagater was used in this case.

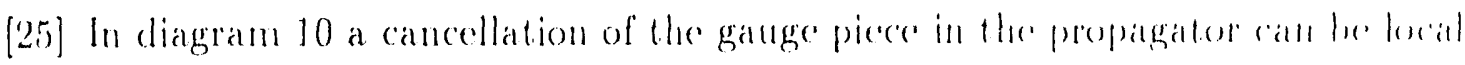
for only one of the photons.

[26i] 'This remark refers only' to non-Abelian gange theories.

[27] Note that the one-loop anomaly does not reguire regularization.

[28] S. J. Brodsky private communication.

[29] M. Kaluza and H. (. Pauli, CAMTP/91.

[3()] Those violations of gauge invariance occur in all ganges, cren if the so-called "gauge principle" is involied [29].

[31) 11. C. Pauli, M. Kaluza, and S. Brodsky, private communication.

[32] In order to regularize this expression, we want to assume that the l'anli Villats condition $\int d \lambda^{2} \rho\left(\lambda^{2}\right)=0$ has been imposed. 
[33] The problem does not occur in the self-energy dingram 1 of Fig. 4.1, because the double instantaneous diagram does not contribute, since the gt current is computed here.

[31) In light-cone gauge the situation is more complicated, due to extra terms of the form

$$
\frac{n k(p-m)+(p-m) k n}{\eta k}+\frac{n\left(p^{2}-m n^{2}\right)}{\eta k} .
$$

Wo see that even single instantaneous graph give rise to a mass subtraction in the a.d. method even though $\delta m$ is gauge invariant.

[35] This is because the difference is independent of the photon mass.

[36] J. Collins, "Renormalization" (Cambridge University Press, ('ambridge 1984), page 60.

[37] This is after multiplication with a term proportional to e from the onc-loop anomaly. 


\section{A HAMILTONIAN FORMULATION OF QED $_{2+1}$ ON THE LIGHT CONE}

\subsection{Introduction}

One of the main advantages of the light-cone quantization in field theory is its manifest invariance under a maximally large subgroup of the Lorentz group (1) which contains even certain boost transformations. The corresponding generators of these "simple" transformations are nondynamical operators, i.e., they do not involve any" interaction terms, Such nondynamical symmetries can be preserved under a wide class of approximations [2], such as, eg., cutofls in the mumber of particles, 'lhis feature greatly simplifies the task of constructing the IIamitonian formulation of a relativistic field theory.

The price to pay for having simple generators of boust transformations is the occurrence of complicated and dynamical generators for certain rotations which implices that angular momentum is not manifestly conserved in the light-cone quantization. We will show that this results in a divergent structure of even supor renomalizable theories.

Rotational invariance, is not a natural symmetry in the light-cone quantization procedure since it mixes longitudinal and transverse degrees of freedom. In particular an improper treatment of the short distance singularitics due to regularization will result in a violation of rotational invariance. In fact most approximations or regular. izations (if infinities are present) will spoil rotational invariance, for rotations which mix the $\underline{x}^{-}=\left(x^{-}, x^{1}, x^{2}\right)$ and $x^{+}$direction [3]. In this chaptere we will concentrate on this aspect.

We will discuss several complementary approaches to this problem. The first, using Pauli..Villars (P-V) regularization, softens the short distance singularitios and thus avoids the cause of the problem, since it regularizes symuntrically in longitu. 
dimal and transverse coordinates. The second appronch starts from the naive light cone quantigation. Any violations of rotational invartance, deg. due to an impropere treatment of the short distance singularitios, are then cancelled by adding cxplicitly rotational noninvariant terms to the light-cone Hamiltonian.

'The resulting regularization and renormalization program has a promi molhing to do with the usual renomalizations of mass and charge. As a matter of fact, while infinite mass and change renormalization are often not necessaty in less than $3+1$ dimensions, the problems which are discenssed here appear in any number of dimensions (except in 1+1, where there are no spatial rotational) |11.

In order to emphasize this point we will mostly work in :-t. I dimentions. This will help separate light-rone specifie divergencess and renormalizations from the usual ones. An extension of the technigues developed here to 3 th d dimensions will he described at the end of this paper.

\subsection{Pauli-Villars regularization of the light-cone quantized Yukawa model}

As a simple example, which exhibits matny of the lighte-cone rentaled poblents, we first consider the lighte-cone guantized Sukawa model,

$$
\left.\dot{L}=\vec{\psi}(i \phi)-m) \psi-\phi(\square)+\lambda^{\prime 2}\right) \phi+\gamma \bar{\psi} \psi(\phi)
$$

in $2+1$ dimensions. It is easy to sturly the violation of rotational intiatiance in this model since it is - in contrast to, e.ge, gange theories in the light-cone gange described by a fully covariant Lagrangian, i.e., event off shell Grent's functions should exhibit covariance. In particular, one should be abte to express the fermion self encrgy in the form

$$
\mathbb{S}\left(p^{\prime \prime}\right)=\left(p_{1}-m\right) f_{1}\left(p^{2}\right)+f_{2}\left(p^{2}\right)
$$




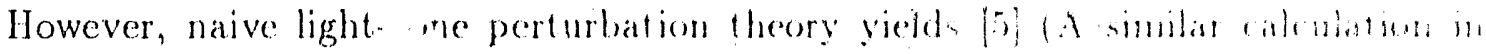

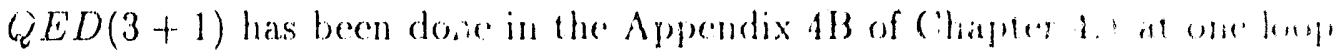

$$
\begin{aligned}
& \operatorname{tr}\left(\Sigma \gamma^{+}\right)=c p^{+} \int_{0}^{1} d x \int_{-\infty}^{\infty} d k_{\perp} \frac{1-x}{\left.\left.x(1-x) p^{2}-m^{2} x-\lambda(1) x\right)\left(k^{2}-x\right)^{\prime}\right)} \\
& -" \lambda-\Lambda " \\
& \operatorname{tr}\left(\Sigma \gamma^{-}\right)=\frac{c}{p^{+}} \int_{0}^{1} d x \int_{-\infty}^{\infty} d k_{\perp} \frac{\frac{m^{2}+\left(p_{1}-k_{1}\right)^{2}}{1-r}}{x(1-x) p^{2}-m^{2} r-\lambda^{2}(1-x) \cdots\left(k_{1} \cdots p_{1}\right)^{2}} \\
& -" \lambda \rightarrow \Lambda "
\end{aligned}
$$

where $c=\gamma^{2} / \pi$. Adding

$$
0=\frac{1}{1-x} \frac{x(1-x) p^{2}-m^{2} x-\lambda^{2}(1-x)-\left(k_{1}-x_{1}\right)^{2}}{x(1-x) p^{2}-m^{2} x-\lambda^{2}(1-x)-\left(k_{1}-x_{1}\right)^{2}} \cdot, \cdot, \cdot \quad \text {, } \quad \therefore
$$

to the integrand in Eq. (5.4) one finds

$$
\begin{aligned}
& \operatorname{tr}\left(\Sigma \gamma^{-}\right)=\frac{c}{p^{+}} \int_{0}^{1} d x \int_{-\infty}^{\infty} d k_{\perp} \frac{x p^{2}+m^{2}-\lambda^{2}+\left(1-x^{2}\right) p_{\perp}^{2}}{x(1-x) p^{2}-m^{2} x-\lambda^{2}(1-x) \cdots\left(k_{\perp}-x p_{\perp}\right)^{2}} \\
& -" \lambda \rightarrow \Lambda " \\
& =\frac{c}{p^{+}} \int_{0}^{1} d x \int_{-\infty}^{\infty} d k_{\perp} \frac{(1-x)\left(p^{2}+p_{\perp}^{2}\right)-\frac{d}{d x}\left[x(1-x) p^{2}-m^{2} x^{2}-\lambda^{2}(1-x)\right]}{x(1-x) p^{2}-m^{2} x-\lambda^{2}(1-x)-\left(k_{\perp}-x p_{\perp}\right)^{2}} \\
& -" \lambda \rightarrow \Lambda " \\
& =\frac{p^{-}}{p^{+}} \operatorname{tr}\left(\Sigma \gamma^{+}\right)-\frac{c \pi}{2 p^{+}}\left[\left(\sqrt{m^{2}}-\sqrt{\lambda^{2}}\right)-" \lambda \rightarrow \Lambda^{\prime \prime}\right] .
\end{aligned}
$$

Obviously two conditions, namely $\int d \lambda^{2} \rho\left(\lambda^{2}\right)$ and $\int d \lambda^{2} \sqrt{\lambda^{2}} \rho\left(\lambda^{2}\right)=0$, are necessary forancel the noncovariant term which implies the need for ar least two P.V. particles. This is rather unpleasant, and perhaps unexpected, since in a manifestly covariant. 


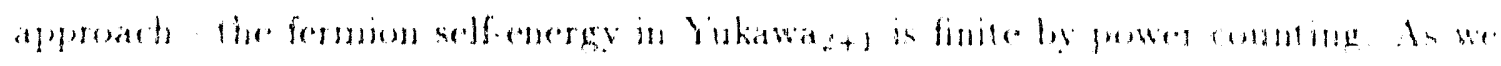

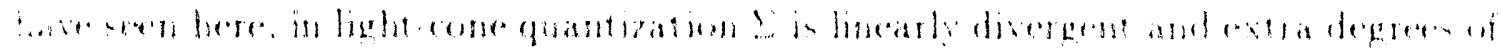

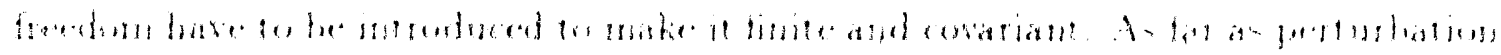

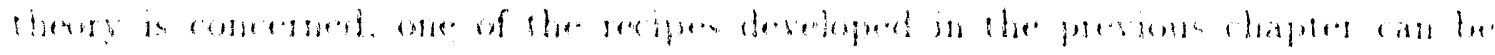

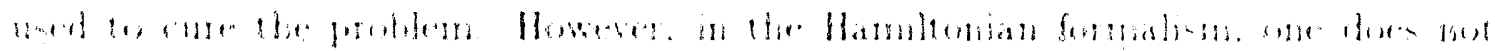

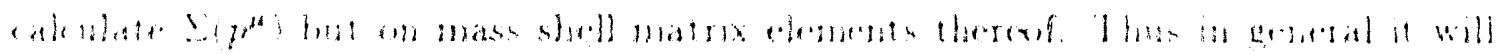

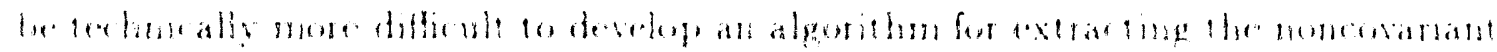

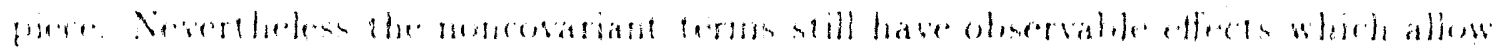

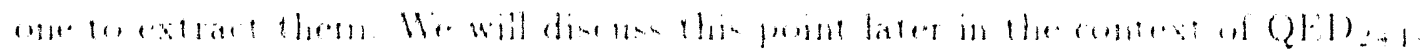

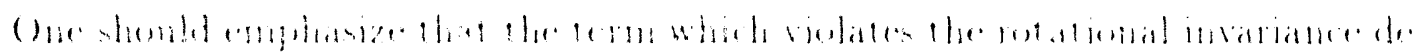

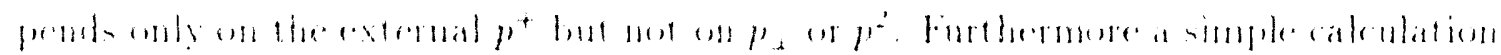

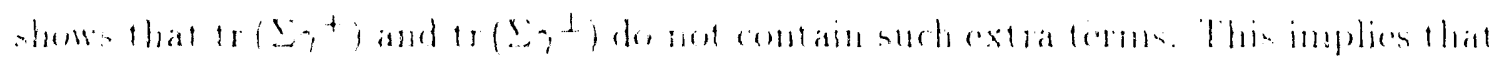
weran write

$$
\sum^{L \prime}\left(p^{\prime \prime}\right)=\sum^{\prime \cdots \prime}\left(p^{\prime \prime}\right)+\text { const } \cdot \frac{2^{+}}{p^{+}}
$$

This is a general result which also holds for higher loops [ii] provided atl nonco. variant terms have been removed for subloops and for other field theories liter e.g. QED) in light-cone gauge. This has various practical conseguences. First one might be able to remove this term by adding a counterterm to the Hamiltonian (i.e., hy changing the mass of the fermion in the kinetic energy term). Secondly this allows one to develop simple subtraction procedures in perturbative calculations to get rid of such terms (see null-subtraction introduced in Chapter 4).

A last point which we are going to make in the context of Yukawa $2+1$ concorns the "over regularization" of the theory. As we mentioned already there are no P-V particles necessary in covariant perturbation theory whereas we needed two of them for a more one-loop treatment. At higher loops the situation becomes a little better, namely one P-V particle is sufficient, (provided subloops are rendered covariant) but. 
it is also necessary in general as the example in Appendix 513 shows. For renomalization theories where P. V regularization poses no extra problems, like (QED) $3+1$, this means that there is no more regularization necessary than one would normally need. However, in non-Abelian gange theories P.V regularization violates gauge invariance and we would have to restore it by further counterterms. We also emphazise, and this can also be read of from the example in Appendix 5B, that dimensional regularization does not take care of the noncovariant terms. The reason for this is that dimensional regularization in the transverse coordinate does not regularize the longitudinal coordinates.

\subsection{Hamiltonian formulation for $\mathrm{QED}_{2+1}$ in the light cone gauge (Pauli-Villars regularization)}

We start our considerations from the QED- Lagrangian in two space and onc-tine dimensions with gauge fixing term $\left(n_{\mu} A^{\mu}=A^{+}\right)$

$$
\mathcal{L}=\mathcal{L}_{\mathrm{Ferm}}+j_{\mu} A^{\mu}-\frac{1}{4} F_{\mu \nu} F^{\mu \nu}-\lim _{\xi \rightarrow \infty} \frac{\xi}{2}\left(n_{\mu} A^{\mu}\right)^{2}
$$

For the purpose of P.-V regularization (as well as if one wants to introduce an IRregulator) it is necessary to specify how to introduce a mass for the A-field. One might be tempted to add just a term like $\left(\Lambda^{2} / 2\right) A_{\mu} A^{\mu}$ to Eq. (5.8). However, since $A_{\mu} A^{\mu}=A^{+} A^{-}-A_{\perp}^{2}=-A_{\perp}^{2}$ (note: $A^{+}=0$ ) this means that only the $\perp$-degrees of freedom become massive whereas the longitudinal degrees of freedom remain massless. In terms of the photon propagator this means

$$
D_{\Lambda}^{\mu \nu}=-\lim _{\xi \rightarrow \infty}\left[\left(k^{2}-\Lambda^{2}\right) g^{\mu \nu}+\xi n^{\mu} n^{\nu}-k^{\mu} k^{\nu}\right]^{-1}=-\frac{g^{\mu \prime}-\frac{k^{\mu} n^{\nu}+k^{\prime \prime} n^{\mu}}{k n}+\frac{\Lambda^{2} n^{\mu} \eta^{\nu}}{(n k)^{2}}}{k^{2}-\Lambda^{2}+i \epsilon}
$$


i.e, even at the tree level, the photon propagator does not vanish for $\Lambda^{2} \rightarrow \infty$ and the "instantaneous" contribution

$$
\lim _{\Lambda \rightarrow \infty} D_{\Lambda}^{\mu \prime \prime}=\frac{n^{\mu} n^{\nu}}{(n k)^{2}}
$$

remains. What one has to do, in addition to adding an $\left(\Lambda^{2} / 2\right) \Lambda_{\mu} A^{\mu}$ term to $\mathcal{L}$, is to introduce a dynamical longitudinal degree of freedom: a scalar field $\phi$ of mass $\Lambda$ which couples with strength $\left(e \Lambda / k^{+}\right)$to the current $j^{+}$, i.e.,

$$
\delta \mathcal{L}_{\text {long }}=-\phi\left(\square+\Lambda^{2}\right) \phi+i \epsilon \Lambda \phi \frac{1}{n^{\mu} \partial_{\mu}} n^{\mu} j_{\mu}
$$

The effect of this scalar field can be absorbed into the photon propangator, yielding

$$
\left.\widetilde{D}_{\Lambda}^{\mu \nu}(\mathrm{eff})=D_{\Lambda}^{\mu \nu}+D_{\Lambda}^{\mu \prime \prime} \text { (longitudinal }\right)=-\frac{g^{\mu \nu}-\frac{n^{\prime \prime} k^{\nu}+n^{\nu} k^{\prime \prime}}{n k}}{k^{2}-\Lambda^{2}} .
$$

Since for on-shell Greens functions the $n^{\prime \prime} k$ terms do not contribute [i], all s-matrix elements should exhibit rot ational invariance oven for finite $\Lambda^{2}$ !

Having specified how to treat the $A$-field we can now proced to construet the Hamiltonian. As a matter of convenience we choose to represent the Hamiltonian using discrete light-cone quantization (DLCQ) [8,9] . Except for the longitudinal ficld this has been done already by $\mathrm{A}$. Tang [10] for $\mathrm{QED}_{3+1}$ so that we do not have to go into the details. For one flavor of fermion $\left(b^{+}=\right.$fermion, $d^{+}=$antifermion $)$ and one massive photon $\left(a^{+}=\right.$transverse photon, $c^{+}=$Iongitudinal photon) one finds in $2+1$ dimensions

$$
H=H_{0}+V_{\text {flip }}+V_{\text {no flip }}+V_{\text {inst phot }}+V_{\text {long }}+V_{\text {inst ferm }}+V_{N O}
$$

where

$$
\begin{aligned}
H_{0}= & \sum_{\underline{p}} \frac{1}{p}\left[\lambda^{2}+\left(\frac{p_{\perp} \pi}{L_{\perp}}\right)^{2}\right]\left[a_{\underline{p}}^{+} a_{\underline{p}}+c_{\underline{p}}^{+} c_{\underline{p}}\right] \\
& +\sum_{s, \underline{n}} \frac{1}{n}\left[m^{2}+\left(\frac{n_{\perp} \pi}{L_{\perp}}\right)^{2}\right]\left[b_{s, \underline{n}}^{+} b_{s, \underline{n}}+d_{s, \underline{\underline{p}}}^{+} d_{s, \underline{\underline{n}}}\right],
\end{aligned}
$$




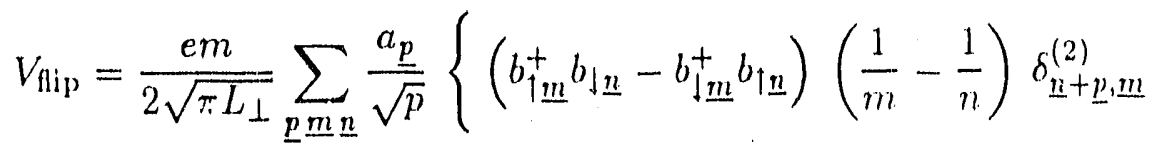

$$
\begin{aligned}
& -\left(d_{1 \underline{m}}^{+} d_{\underline{\underline{n}}-}-d_{\mathfrak{I}_{\underline{m}}}^{+} d_{\uparrow \underline{n}}\right)\left(\frac{1}{m}-\frac{1}{n}\right) \delta_{\underline{\underline{n}}+\underline{p}, \underline{m}}^{(2)}
\end{aligned}
$$

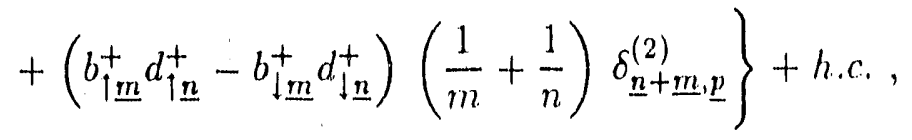

$$
\begin{aligned}
& V_{\text {no flip }}=\frac{c}{2 L_{\perp}} \sqrt{\frac{\pi}{L_{\perp}}} \sum_{g, p, \underline{m}, \underline{m_{n}}} \frac{a_{\underline{p}}}{\sqrt{p}}\left(2 \frac{p_{\perp}}{p}-\frac{n_{\perp}}{n}-\frac{m_{\perp}}{m}\right)
\end{aligned}
$$

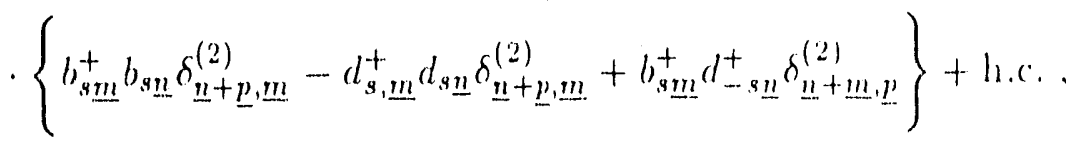

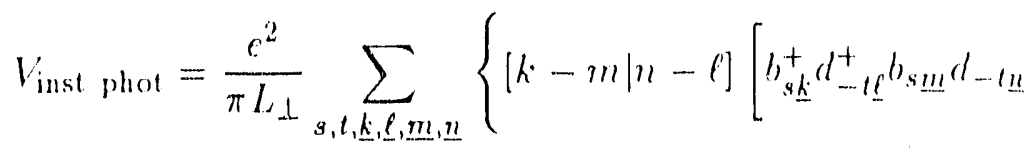

$$
\begin{aligned}
& \left.-\frac{1}{2} b_{s \underline{k}}^{+} b_{t \underline{\underline{e}}}^{+} b_{s \underline{\underline{m}}} b_{t \underline{\underline{n}}}-\frac{1}{2} d_{s \underline{\underline{k}}}^{+} d_{t \underline{t}}^{+} d_{s \underline{\underline{m}}} d_{t \underline{\underline{u}}}\right]
\end{aligned}
$$

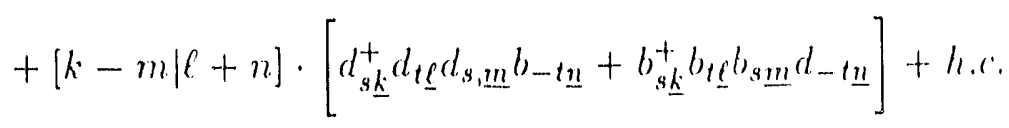

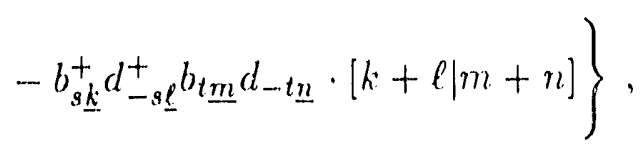

$$
\begin{aligned}
& V_{\text {long }}=\frac{e \lambda}{\sqrt{\pi L_{\perp}}} \sum_{g, \underline{k}, \underline{\ell}, \underline{\underline{m}}} \frac{1}{\ell^{3 / 2}} c_{\ell} \cdot\{ \\
& \left.\left(b_{s \underline{k}}^{+} b_{s, \underline{m}}-d_{\underline{3} \underline{k}}^{+} d_{s, \underline{m}}\right) \delta_{\underline{\underline{k}, \underline{m}+\underline{\ell}}}^{(2)}+b_{s \underline{\underline{k}}}^{+} d_{-s \underline{\underline{m}}}^{+} \delta_{\underline{\underline{l}}, \underline{\underline{k}}+\underline{\underline{m}}}^{(2)}\right\}+h . c .
\end{aligned}
$$




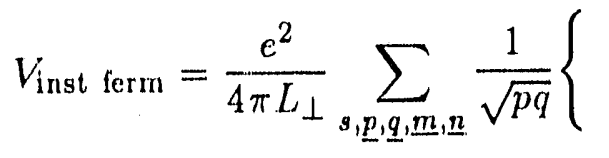

$$
\begin{aligned}
& a_{\underline{p}}^{+} a_{\underline{q}}\left[b_{s \underline{m}}^{+} b_{\underline{s} \underline{\underline{n}}}+d_{s_{\underline{m}}}^{+} d_{\underline{\underline{\underline{n}}}}\right] \cdot \mid\{\{r+m\{q+n\}-\{p-n \mid q-m\}] \\
& \left.+a_{\underline{p}}^{+} a_{\underline{q}}^{+} b_{s \underline{m}} d_{-s \underline{n}} \cdot\{p-m \mid \cdot q+q\}\right\}+ \text { h.c. } \\
& +a_{\underline{p}}^{+} a_{\underline{q}} b_{s \underline{m}} d_{-s \underline{\underline{n}}} \cdot[\{p-m \mid q+n\}-\{p-n \mid q+m\}]+h . c . \\
& \left.+a_{\underline{\underline{p}}, \underline{q} \underline{\underline{q}}}\left[b_{\underline{\underline{m}}}^{+} b_{s \underline{\underline{n}}}+d_{s \underline{\underline{m}}}^{+} d_{s \underline{\underline{n}}}\right] \cdot\{p+n \mid-q+m\}+h . c .\right\}
\end{aligned}
$$

Here

$$
\begin{aligned}
p, q & =2,4,6, \ldots \\
k, l, m, n & =1,3,5, \ldots \\
p_{\perp}, q_{\perp}, k_{\perp}, l_{\perp}, m_{\perp}, n_{\perp} & =0, \pm 1, \pm 2, \ldots \\
s, t & =\uparrow, \downarrow
\end{aligned}
$$

$V_{N O}$ represents the normal ordering termis which are part of the $\mathcal{O}\left(c^{2}\right)$ contributions to the self-energies. Since they arise from instantaneous interactions th ey are independent of particle masses and thus vanish in P.-V regularization [11].

We leave the explicit construction of the P.-V regularized Hamiltonian to the Appendix. For perturbative calculations we will weight the contributions frem the various electrons and photons (physical and $\mathrm{P}-\mathrm{V}$ ) with coefficients $c_{i}^{2}$ and $c_{j}^{2}$ which are 
later determined such that all unwanted terms vanish. E.g., the $\mathcal{O}\left(e^{2}\right)$ contributions to the self-energy of a transverse photon with momentum $\underline{p}$ are $\left(\hat{p}_{\perp}=p_{\perp}\left(\pi / L_{\perp}\right)\right)$ :

$$
\begin{aligned}
\delta E_{\underline{p}}^{\mathrm{trans}}= & \frac{e^{2}}{4 \pi L_{\perp}} \frac{1}{p} \sum_{i} c_{i}^{2} \sum_{n} \\
& \times \frac{m_{i}^{2}\left(\frac{1}{n}+\frac{1}{p-n}\right)^{2}+\left(\frac{2 \hat{p}_{\perp}}{p}-\frac{\hat{n}_{\perp}}{n}-\frac{\hat{p}_{\perp}-\hat{n}_{\perp}}{p-n}\right)^{2}}{\frac{\lambda^{2}+\widehat{p}_{\perp}^{2}}{p}-\frac{m_{\perp}^{2}+\widehat{n}_{\perp}^{2}}{n}-\frac{m_{i}^{2}+\left(\hat{p}_{\perp}-\widehat{n}_{\perp}\right)^{2}}{p-n}} .
\end{aligned}
$$

In order to obtain a finite results in the continuum limit we have to require $\sum_{i} c_{i}^{2}=0$. This allows us to simplify the numerator by using the replacement $\left(\widehat{n}_{\perp}-\widehat{p}_{\perp L}(n / p)\right)^{2} \rightarrow$ $-m_{i}^{2}+\lambda^{2} / p(1 / n+1 / p-n)^{-1}$, i.e.,

$$
\begin{aligned}
\delta E_{\underline{p}}^{\text {trans }}= & \frac{e^{2}}{4 \pi L_{\perp}} \frac{1}{p} \sum_{i} c_{i}^{2} \sum_{n} \\
& \times \frac{4 m_{i}^{2}+\lambda^{2} \frac{(p-2 n)^{2}}{p^{2}}}{n(p-n)\left\{\frac{\lambda^{2}}{p}-\left(\frac{1}{n}+\frac{1}{p-n}\right)\left[\left(\hat{n}_{\perp}-\hat{p}_{\perp} \frac{n}{p}\right)^{2}+m_{i}^{2}\right]\right\}} \\
= & \frac{e^{2}}{4 \pi L_{\perp}} \frac{1}{p} \sum_{i} c_{i}^{2} \\
& \times-\frac{4 m_{i}^{2}+\lambda^{2}\left[1-8 \frac{n}{p}\left(1-\frac{n}{p}\right)\right]}{n(p-n)\left\{\frac{\lambda^{2}}{p}-\left(\frac{1}{n}+\frac{1}{p}-n\right)\left[\left(\hat{n}_{\perp}-\hat{p}_{\perp} \frac{n}{p}\right)^{2}+m_{i}^{2}\right]\right\}} \\
& +\delta E_{\underline{p}}^{\text {long }},
\end{aligned}
$$

where we have already separated the self-energy of a longitudinal photon

$$
\delta E_{\underline{p}}^{\text {long }}=\frac{e^{2}}{4 \pi L_{\perp}} \frac{1}{p} \sum_{i} c_{i}^{2} \sum_{n} \frac{4 \lambda^{2} / p^{2}}{\frac{\lambda^{2}}{p}-\left(\frac{1}{n}+\frac{1}{p-n}\right)\left[\left(\widehat{n}_{\perp}-\widehat{p}_{\perp} \frac{n}{p}\right)^{2}+m_{i}^{2}\right]}
$$


In the continuum limit the self energies of longitudinal and transverse photons must, be equal-otherwise rotational invariance is broken. To analyze this condition further we transform this term into an integral

$$
\begin{aligned}
\delta E^{\text {trans }}-\delta E^{\text {long }} & \rightarrow \frac{e^{2}}{4 \pi^{2}} \frac{1}{p} \sum_{i} c_{i}^{2} \int_{0}^{1} d x \int_{-\infty}^{\infty} d k_{\perp} \frac{4 m_{i}^{2}+\lambda^{2}[1-8 x(1-x)]}{\lambda^{2} x(1-x)-m_{i}^{2}-k_{\perp}^{2}} \\
& =-\frac{e^{2}}{\pi} \frac{1}{p} \sum_{i} c_{i}^{2} \sqrt{m_{i}^{2}}
\end{aligned}
$$

and our second P-V condition has to be $\sum_{i} c_{i}^{2} \sqrt{m_{i}^{2}}=0$.

We have performed similar calculations for the on-shell self-energy of an elertron. Since this is a gange invariant quantity we can reguire that our calculation in light-cone gauge and light-cone quantization repsoduces the covariant result ohtained in Feyman gange and $2+1$ dimensional symmetrical integration. An alternative approach - which will be elaborated in more detail in the next section is to calculate the one-loop corrections to the Compton cross section and comprate with well known results. Both methods lead to the sane condition, namely

$$
\sum_{j} c_{j}^{2}=0 \quad \sum_{j} c_{j}^{2} \sqrt{\lambda_{j}^{2}}=0
$$

For practical calculations it is useful to reduce the number of $P$ V conditions. To achicve this one can add a counterterm to the Hamiltonian which cancels those terms which are multiplied by $c_{i}^{2} \sqrt{m_{i}^{2}}$ and $c_{j}^{2} \sqrt{\lambda_{j}^{2}}$ in the self-cnergies of photons and electrons respectively. At one loop this reduces-aby construction the number of P.-V conditions required. However, and this is a highly nontrivial result, mumerical calculations of the self-energies as well as the example in Appendix $5 B$ show that this is also true for higher loops, i.e., the second P-V particle is only necessary at one loop. Once we avoid it by adding a suitable one-loop countertim there is only one P-V particle needed at two loops and most probably also for higher loops. 
There might be various reasons for this special behavior at one loop. First of all there are ambiguities in how to treat normal ordering divergences which are of $\mathcal{O}\left(e^{2}\right)$ and contribute only to the one-loop selfenergies. Secondly, power counting in light-cone coordinates is different from the usual covariant power counting [12]. One has to count separately powers in $k_{\perp}$ and $1 / k^{+}$in order to properly estimate the degree of divergence. Here it turns out that the strongest divergence (e.g., at quadratic $k_{\perp}$ divergence in $3+1$ divergences) occurs only at the one-loop level. 'The situation here is similar to scalar QED in equal-time quantization [13].

\subsection{Renormalization using noncovariant counterterms}

QED2+1 is super renomalizahle and only two graphs are superficially divergent in leynman perturbation theory the one and twe-loop vacumm polatration are finite if gange invariant regularization is used.). Howerer, the presence of terms which break rotational invariance has forced us to introduce four P V particles (two photons and two electrons), i.e., the fork space content of the theory has increased considerably. Fen after calculating the one-loop counterterums hy hand one has to deal with one P-V photon and one P-V electron, i.e., the mumber of degrees of freedom still increases by a factor of four compared to the muregularized therery.

Furthermore practical calculations require in general some approximations which in general lead to further violations of rotational invariance [14]. In this work wo deal only with those violations of rotational invariance which are indued by an improper treatment of the high energy degrees of freedom (large $k_{\perp}$, small al) if an P..V regularization, or anything equivalent, is applied. ('The methods, which we are going to develop for the latter problem, should, however, also be applicable for approximation-induced effects.)

Using the light-cone power counting rules one show's that light-cone QED in $3+1$ and $2+1$ dimensions is renormalizable [11]. This implies that the violations 
of rotational invariance (which in our case are induced by an improper handling of arbitrarily high energies) can be compensated by a redefinition of terms in the Hamiltonian. In general such a renormalization procedures can be quite lengthy since, at least in principle, the $e^{-}$masses which appear in the kinetic cnergy and in the vertex, the various $e^{-}$charges and the various photon masses can all require different renormalizations, i.e., instead of three renormalization constants $(m, \lambda, e)$

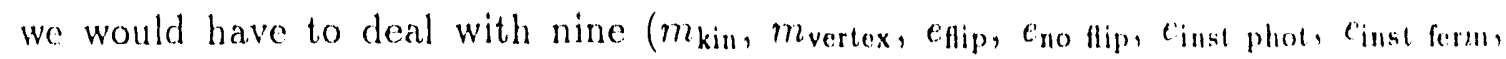
$\left.\lambda_{\text {long }}, \lambda_{\text {trans }}, \lambda_{\text {vertex }}\right)$. However, practical calculations have shown that violations of rotational invariance in $L C$ gauge occur only in two-point functions and there only in a very specific form $[15]$, namely

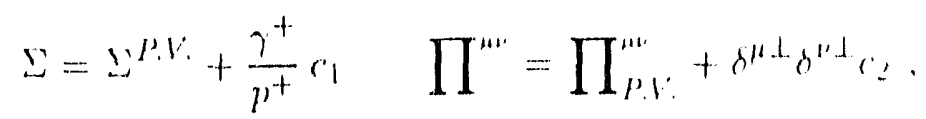

for electron and photon self-energies respectively. i.e.the deviations from the pl' regularized results. which lead to rotational invariant observaliles can be param

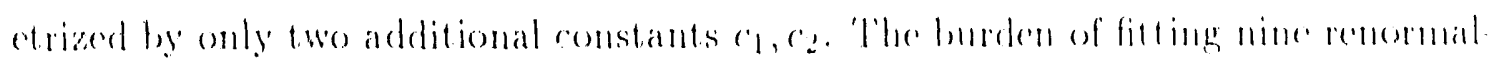
ization constants has thus beren reduced to fitting five $[16 i]$. In pradice one adels two extra connterterms

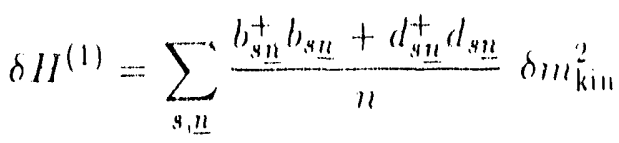

$$
\begin{aligned}
& \delta H^{(2)}=\sum_{\underline{p}} \frac{a_{\underline{p}}^{+} a_{\underline{p}}}{p} \delta \lambda_{\text {irans }}^{2}
\end{aligned}
$$

to the Hamiltonian and adjusts $\delta m_{\mathrm{kin}}^{2}$ and $\delta \lambda_{\text {irans }}^{2}$ such that rotational invariance is restored (this point will be discussed below). 'The next step, which is not necessary' in $9 \mathrm{HD}_{2+1}$, would then be the usual mass and charge renormalization [17] .

The constants $\delta m_{\text {kin }}^{2}$ and $\delta \lambda_{\text {trans }}^{2}$ are determined as follows. Fixing $\delta \lambda_{\text {trans }}^{2}$ is rather easy: one diagonalizes the Hamiltonian (within some approximations like eg., cutoff in Fock space) for a given $\delta \lambda_{\text {trans }}^{2}$ and compares the physical masses (eigenvalues 
of the Hamiltonian) of longitudinal and transverse photons, $\delta \lambda_{\text {irans }}^{2}$ is then luncel until these eigenvalues coincide.

For $\delta m_{\text {kin }}^{2}$ two methods are suggested. The first method is based on the fact that instantaneous $e^{-}$exchange becomes singular for small $p^{+}$transfer (e.g., in (ompton back scattering). This is of course an unphysical singularity which has to be cancelled by noninstantaneous $e^{-}$exchange. At tree level it is crucial for the cancellation that the kinetic mass of an electron $\left[m\right.$ in $\left.H_{0}(3.7)\right]$ equals the vertex mass $[m$ in $\left.V_{\text {flip }}(3.8)\right]$. At one loop the interaction will renomalize $m_{\text {kin }}$ and $m_{\text {vertex }}$ differently and one can easily convince oneself that the cancellation will be spoiled unless different renormalization comberterms for mekn and mvertex are used. 'This defines already the renomalization procedure, namely tuning man math finiteness of the Compton back scattering amplitude for zero $p^{+}$transfer is achiceded.

The serond method uses the degeneracy of the positrentum spectrum dere to rotational invariance. A glance at the Hamiltonian, Eq. (5.13) show's that, for zero

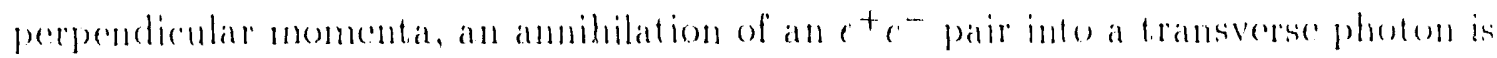
pessible if and only if both have a paralled spin but not for the $4=1,5:=0$ state. Another annibilation process is possible via longitudinal or instantancous photons but only from the $S=1, S_{z}=0$ state. In the first catse the vertex mass apperats whereas in the second it does not. For degeneracy of the th: $=0, \pm 1$ stales it is important that both interactions have the same strength. Again this is achieved al tree level by choosing $m m_{\text {kin }}=m_{\text {vertex }}$ but if loops are taken into account the condition changes. Degeneracy of the $S_{z}=0, \pm 1$ states in the gromud state of positronimm ciun thus be used as a renormalization condition.

The first method seems to be superior from a practical point of view, since it requires to look at the $e^{-\gamma}$ system only and not at $e^{-} e^{+} \gamma$ states as for the second method. However, from a practical point of view we are interested in the positronium spectrum, i.e., we diagonalize the Hamiltonian. The second methods thus requires 
only little effort to implement-namely, diagonalizing $H$ for two spin configuralions and repeating this a few times (to fit sme iteratively). Furthermore, and this will also be of practical importance, the renormalization constants will thus be evaluated antomatically to the same loop order and with the same approximations as the actual positronium calculations are done.

\subsection{Extension to $3+1$ Dimensions}

for those theories considered in this work (Yukawa and QPED) an extension to $3+1$ dimensions is straightforward. The only difference will be that mese cenflicients have to be renomalized and that there will be in general an infinite remomalization.

In practice the following steps have to be performed. If one witnts lo render all

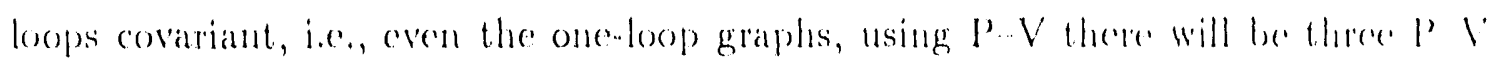
conditions for photons and electrons, namely [18]

$$
\begin{array}{r}
\int d \lambda^{2} \rho\left(\lambda^{2}\right)=0 \\
\int d \lambda^{2} \lambda^{2} \rho\left(\lambda^{2}\right)=0 \\
\int d \lambda^{2} \lambda^{2} \log \lambda^{2} \rho\left(\lambda^{2}\right)=0
\end{array}
$$

which is awkward from a numerical point of view. Thus one should only use the improved version of the P-V approach, where the onc-loop counterterms are constructed "by hand" and only one P-V condition has to be imposed for higher loops. The aumber of degrees of freedom will thus be the same as in a covariant approach (c.g., euclidean integration) with P. V regularization. The method of noncovariant. counterterms might also be very useful. For example, if one uses a kinetic energy cutoff further violations of rotational invariance are induced. The algorithun deseribed in Section 4 would automatically remedy this without further effort. 
The extension to nomabelian gange theories is not as straightorward. All mothods discussed in this work violate local gange invariance at least in intermedialo steps. For QED this is not a problem since, eg., the P'..V regularization preservest the Ward identities. In QCD this is not the case and one has lo add furthor ganger breaking counterterms which restore gange invariance [19].

\subsection{Summary and conclusion}

Naive light-cone quantization without careful regularization violates rotational invariance. In theories with a covariant hagrangian we have denomstraled this hes investigating the covariant structure of self-energies. In the case of a memevarian laggangian (QLe) in the light-cone gange) the Lorentz transformation propertices of Cireen's functions are nontrivial and therefore possible viobations of laneme invari ance alle not obvious.

However, these offects must show up in the calculation of physical processuses. 'la

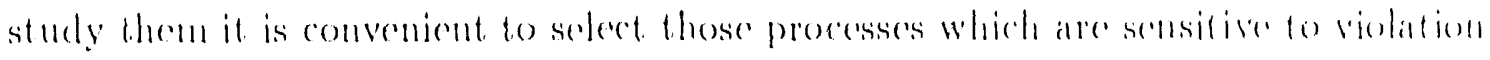

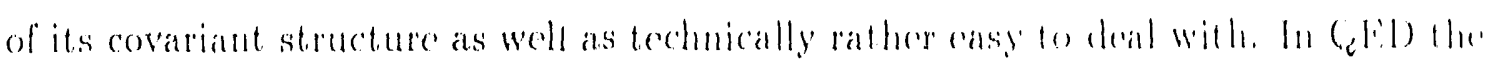

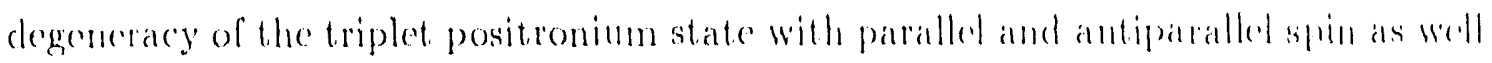
as ('ompton back scattering are such processes.

The violation of rotational invariance is mot limited to one loop, allhought unte might expect this since normal-ordering ambiguitics arise only in onf-loop selfenorgies. In fact, muless regularized properly, the nommal ondering contribulions leat to violation of rotational symmetry. However, those torms are not the only sondere of violations of this kind as our explicit two-loop calculations show. 'The indurent divergences are less severe there, though.

We have discussed from two basic methods to restore rotalional invariance, the Pauli-Villars method and the method of noncovariant comberterms. Buth methouts seem to recuire a large number of additional degrees of frecelom or commerterms. 
However, because of the specific structure of rotational invariage violation in light cone quantization-the worst problems are restricted to one loop and only certain components of two point functions (the $\gamma^{+}$component of the fermion self energy and the $\perp .1-$ components of the vacumm polarization) are affected. This allows us to optimize these nethods considerably. We give analytic expressions for one-loop comnterterns. As a result the P.V. approach then recuires only one ghost per particte to offset the violations of rotational symmetry at higher loops.

The mothod of noncovariant counterterms requires only two additional combterterms (compared to a manilest covariant approach), namely a mass form for transwerse photons and an additional correction to the formion mass term which appears at spin flip photon-electron vertices. 'To fix the additional contitants ome hats

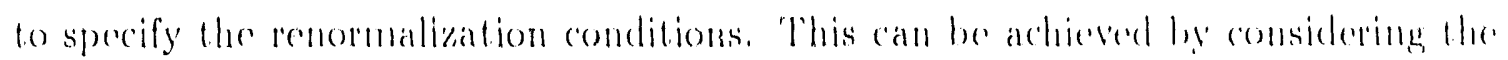
regenerate ground state of positronimu as wedl as the degeneracy of the longitudinal and transwerse photons. 



\subsection{Appendix 5A: The Pauli-Villars regularized Hamiltonian for $\mathrm{QED}_{2+1}$}

As discussed in the section about Pauli--Villars regularization, one Pauli-Villars condition

$$
\begin{aligned}
& \int d m^{2} \rho_{e}\left(m^{2}\right)=0 \\
& \int d \lambda^{2} \rho_{\gamma}\left(\lambda^{2}\right)=0 .
\end{aligned}
$$

for electrons and photons respectively is sufficient to guarantec covariant regularization in all calculations beyond one loop-provided all onc-loop subgraphs have been rendered covariant (e.g., by constructing the necessary one-loop counterterms). (Ine can easily convince oneself that the sum rules (5.30) and (5.31) can be achieved by introducing one additional electron and photon field respectively which are quan. tized with the wrong metric. One way to do so in practice is to introduce an extrat factor of $\sqrt{-1}$ for all heavy photon vertices and another factor of $\sqrt{-1}$ for all heal?! electron pair creation and annihilation vertices. In addition the heavy electron lats to be quantized as a boson.

In practice this implies

$$
H^{\mathrm{PV}}=H_{0}+V_{\text {flip }}+V_{\text {no flip }}+V_{\text {long }}+V_{\text {inst ferm }}+V_{1 \text { loop }}
$$

where 


$$
\begin{aligned}
H_{0}= & \sum_{\underline{p}} \frac{1}{p}\left[\left(\frac{p_{\perp} \pi}{L_{\perp}}\right)^{2}+\lambda^{2}\right] \cdot\left[a_{\underline{p}}^{+} a_{\underline{p}}+c_{\underline{p}}^{+} c_{\underline{p}}\right] \\
& +\sum_{s \underline{\underline{n}}} \frac{1}{n}\left[\left(\frac{n_{\perp} \pi}{L_{\perp}}\right)^{2}+m_{e}^{2}\right]\left[b_{s, \underline{\underline{n}}}^{+} b_{s, \underline{n}}+d_{s, \underline{n}}^{+} d_{s, \underline{n}}\right] \\
& +\sum_{\underline{p}} \frac{1}{p}\left[\left(\frac{p_{\perp} \pi}{L_{\perp}}\right)^{2}+\Lambda^{2}\right]\left[A_{\underline{p}}^{+} A_{\underline{p}}+C_{\underline{p}}^{+} C_{\underline{p}}\right] \\
& +\sum_{s \underline{\underline{n}}} \frac{1}{n}\left[\left(\frac{n_{\perp} \pi}{L_{\perp}}\right)^{2}+M^{2}\right]\left[B_{s, \underline{n}}^{+} B_{s, \underline{n}}+D_{s m \underline{\underline{n}}}^{+} D_{s, \underline{n}}\right]
\end{aligned}
$$

$$
\begin{aligned}
& V_{\text {fip }}=\frac{e}{2 \sqrt{\pi L_{\perp}}} \sum_{\underline{p} \underline{m n}} \frac{\left(a_{\underline{p}}+i A_{\underline{p}}\right)}{\sqrt{p}}\{ \\
& \times\left[m_{e}\left(b_{\uparrow \underline{m}}^{+} b_{\lfloor\underline{\underline{n}}}-b_{\lfloor\underline{m}}^{+} b_{\uparrow \underline{\underline{n}}}\right)+M\left(B_{\uparrow \underline{\underline{m}}}^{+} B_{\lfloor\underline{\underline{n}}}-B_{\lfloor\underline{\underline{m}}}^{+} B_{\uparrow \underline{\underline{n}}}\right)\right]\left(\frac{1}{m}-\frac{1}{n}\right) \delta_{n+p, m}^{(2)}
\end{aligned}
$$

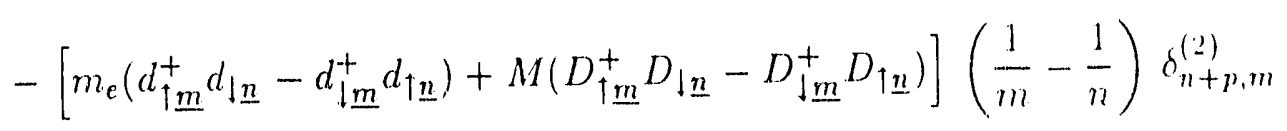

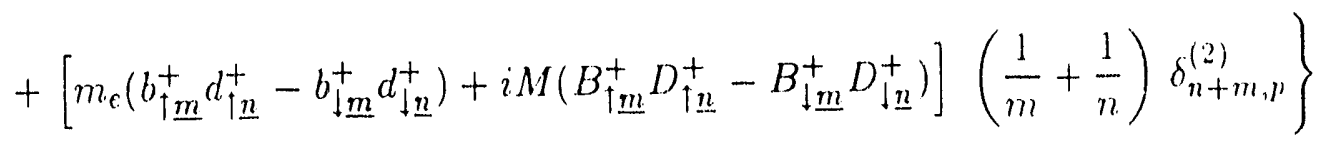

$$
\begin{aligned}
& \text { + "h.c." }
\end{aligned}
$$

$$
\begin{aligned}
& V_{\text {no flip }}=e \sqrt{\frac{\pi}{L_{\perp}}} \frac{1}{2 L_{\perp}} \sum_{s \underline{p} \underline{\underline{m}}} \frac{a_{\underline{\underline{p}}}+i A_{\underline{p}}}{\sqrt{p}}\left(\frac{2 p_{\perp}}{p}-\frac{n_{\perp}}{n}-\frac{m_{\perp}}{m}\right) \\
& \times\left\{\left(b_{s \underline{m}}^{+} b_{s \underline{\underline{n}}}+B_{s \underline{\underline{m}}}^{+} B_{s \underline{\underline{n}}}\right) \delta_{n+p, m}^{(2)}-\left(d_{s \underline{\underline{m}}}^{+} d_{s \underline{\underline{n}}}+D_{s \underline{\underline{m}}}^{+} D_{s \underline{\underline{\underline{n}}}}\right) \delta_{n+p, m}^{(2)}\right. \\
& \left.+\left(b_{s \underline{\underline{m}}}^{+} d_{-s \underline{\underline{n}}}^{+}+i B_{s \underline{\underline{m}}}^{+} D_{-s \underline{\underline{n}}}^{+}\right) \delta_{p, n+m}^{(2)}\right\}+ \text { "h.c." }
\end{aligned}
$$




$$
\begin{aligned}
& V_{\text {long }}=\frac{e}{\sqrt{\pi L_{\perp}}} \sum_{s \underline{k \underline{p} \underline{m}}} \frac{1}{p^{3 / 2}}\left(\lambda c_{\underline{p}}+i \Lambda C_{\underline{\underline{p}}}\{\right. \\
& \times\left[b_{s \underline{k}}^{+} b_{s \underline{m}}+B_{s \underline{k}}^{+} B_{s \underline{m}}\right] \delta_{m+p, k}^{(2)}-\left[d_{s \underline{k}}^{+} d_{s \underline{m}}+D_{s \underline{k}}^{+} D_{s \underline{m}}\right] \delta_{m+p, k}^{(2)} \\
& \left.+\left[b_{s \underline{k}}^{+} d_{-s \underline{m}}^{+}+i B_{s \underline{k}}^{+} D_{-s \underline{m}}^{+}\right] \delta_{k+m, p}^{(2)}\right\} \\
& V_{\text {inst ferm }}=e^{2} \frac{1}{4 \pi L_{\perp}} \sum_{g} \sum_{p q m n} \frac{1}{\sqrt{p q}}\left\{\left(a_{\underline{p}}^{+}+i A_{\underline{p}}^{+}\right)\left(a_{\underline{p}}+i A_{\underline{p}}\right)\right.
\end{aligned}
$$

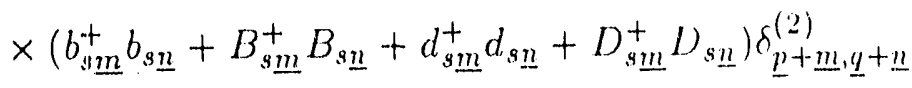

$$
\begin{aligned}
& \times(\{p+m \mid q+n\}=\{p-n \mid q-m\}) \\
& -\left(a_{\underline{p}}^{+}+i A_{\underline{p}}^{+}\right)\left(a_{\underline{q}}^{+}+i A_{\underline{q}}^{+}\right)\left(d_{-s_{\underline{n}}} b_{\underline{\underline{I} \underline{n}}}+i D_{-s_{\underline{n}}} B_{s \underline{\underline{n}}}\right) \\
& \times \delta_{\underline{p}+\underline{q}, \underline{m}+\underline{n}}^{(2)}\{p-m \mid-q+n\}+\text { "h.c." }
\end{aligned}
$$

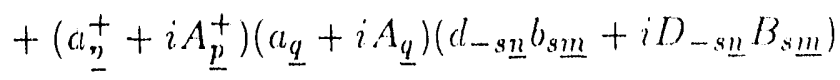

$$
\begin{aligned}
& \times \delta_{\underline{p}, \underline{q}+\underline{m}+\underline{n}}^{(2)}(\{p-n \mid q+m\}-\{p-m \mid q+n\})+\cdot \text { h.c." } \\
& \left.+\left(a_{\underline{p}}+i A_{\underline{p}}\right)\left(a_{\underline{q}}+i A_{\underline{q}}\right)\left(b_{s \underline{m}} b_{s \underline{\underline{n}}}+B_{s \underline{\underline{m}}}^{+} B_{s \underline{\underline{w}}}+d_{s \underline{\underline{m}}}^{+} d_{s \underline{\underline{\eta}}}+D_{s \underline{\underline{m}}}^{+} l\right)_{s \underline{\underline{m}})}\right)
\end{aligned}
$$

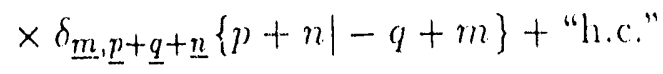




$$
\begin{aligned}
& V_{1 \text { loop }}=\frac{e^{2}}{4 \pi L_{\perp}} \sum_{p} \frac{a_{\underline{p}}^{+} a_{\underline{p}}}{p}\left\{\sum_{i, n} \frac{\lambda^{2}\left[8 \frac{n}{p}\left(1-\frac{n}{p}\right)-1\right]-4 m^{2}}{\frac{\lambda^{e}+\widehat{p}_{\perp}^{2}}{p}-\frac{m^{2}+\widehat{n}_{\perp}^{2}}{n}-\frac{m^{2}+\left(\hat{p}_{\perp}-\widehat{n}_{\perp}\right)^{2}}{p-n}}-" m \rightarrow M "\right\} \\
& +" a_{\underline{p}}^{+} a_{\underline{p}} \rightarrow A_{\underline{p}}^{+} A_{\underline{p}}, \lambda^{2} \rightarrow \Lambda^{2 "}+\frac{e^{2}}{4 \pi L_{\perp}} \sum_{n, s} \frac{\left[b_{s \underline{\underline{n}}}^{+} b_{s \underline{\underline{n}}}+d_{\underline{s} \underline{\underline{n}}}^{+} d_{\underline{\underline{n}}}\right]}{n} \\
& \times\left\{\sum_{p} \frac{\lambda^{2}-s\left[m^{2}+\widehat{n}_{\perp}^{2}\right] \cdot \frac{p}{n}+2 \widehat{n}_{\perp} \widehat{p}_{\perp}}{\frac{m^{2}+\widehat{n}_{\perp}^{2}}{n}-\frac{\lambda^{2}+\widehat{p}_{\perp}}{p}-\frac{m^{2}+\left(\hat{n}_{\perp}-\widehat{p}_{\perp}\right)^{2}}{n-p}} \frac{1}{p(p-n)}-" \lambda^{2} \rightarrow \Lambda^{2 "}\right\} \\
& +" b^{+} b \rightarrow B^{+} B, d^{+} d \rightarrow D^{+} D, m^{2} \rightarrow M^{2} " \text {. }
\end{aligned}
$$

The conventions are the same as in Eqs. (5.13)-(5.19). $a_{\underline{p}}, A_{\underline{q}}, c_{\underline{p}}, C_{\underline{q}}, B_{s, \underline{m}}, D_{s, \underline{n}}$ obey usual boson commutation relations, $b_{s, \underline{m}} d_{s, \underline{n}}$ fermion anticommutation relations. "h.c." indicates Hermitian conjugation only for field operators--not for 6 numbers, i.e., $i A_{\underline{p}}+$ "h.c." $=i A_{\underline{p}}+i A_{\underline{p}}^{+}$. Of course $H$ is thus not hermitian but this should not influence unitarity below the production threshold for the heavy photons and electrons. There is no instantaneous photon exchange term since those terms cancel among the light and heavy photons [20]. The one-loop counterterms have been constructed such that they, together with one-loop corrections induced by $H$, avoid all one-loop self-energies which would be proportional to $\int d m^{2} \rho\left(m^{2}\right) \sqrt{m^{2}}$ or $\int d \lambda^{2} \rho\left(\lambda^{2}\right) \sqrt{\lambda^{2}}$ in the continuum limit. Without the one-loop counterterms nore Pauli-Villars particles would be necessary to make all such terms vanish. 


\subsection{Appendix 5B: The two-loop self energy in Yukawa $D_{\perp}+2$}

In light-cone pertubation theory (LCPTh) the strong divergences (quadratic in $3+1$ ) occur at the one-loop level. Thus one might be tempted to expect that the violations of rotational invariance occur also only at ore loop. This is not true as the following simple example shows.

We consider a massless fermion coupled to a massive scalar boson via a Yukawa interaction term. As a specific example we evaluate explicitly the rainbow graph (Fig. 5.1) contribution to the $\gamma^{+}$component of the one-shell fermion self-energy. If we choose vanishing $p_{\perp}$ for the incoming electron, i.e., $p_{\mathrm{in}}^{-}=p_{\perp}^{2} / p^{+}=0$, it follows from (2.2) that this component should be zero.

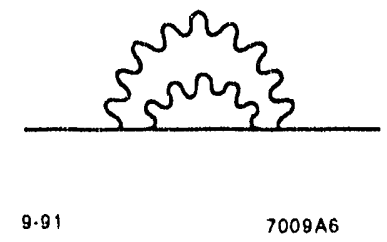

Figure 5.1. Rainbow diagram contribution to the two-loop fermion self-energ! in the Yukawa model.

In order to separate one-loop and two-loop effects we allow the masse's of the inner $(\lambda)$ and the outer boson $(\Lambda)$ in the diagram to be different from each of herp. This also makes it easy to regularize the inner loop "sufficiently" while leaving the outer loop unregularized for the moment. Applying LC.PTh one casily finds [21] (u1) to the same constants)

$$
\Sigma\left(p^{\mu}\right)=C \int d^{D_{\perp}} k_{\perp} \int_{0}^{1} \frac{d x}{x(1-x)^{2}} \frac{p_{1}}{\frac{k_{\perp}^{2}}{1-x}+\frac{k_{\perp}^{2}+\lambda^{2}}{x}} I^{1 L O O P^{\prime}}\left(p_{1}\right) \frac{p_{1}}{\frac{k_{\perp}^{2}}{1-x}+\frac{k_{\perp}^{2}+\lambda^{2}}{x}}
$$

where

$$
p_{1}^{+}=p^{+}(1-x) \quad p_{1}^{-}=-\frac{k_{1}^{2}+\lambda^{2}}{x p^{+}}
$$


and

$$
I^{1 L O O P}\left(p_{1}\right)=\frac{p_{1}}{1-x} \int d^{D_{\perp}} q_{\perp} \int_{0}^{1} \frac{d y}{y(1-y)} \int d \Lambda^{2} \frac{(1-y) \rho\left(\Lambda^{2}\right)}{p_{1}^{-}-\frac{q_{\perp}^{2}+\Lambda^{2}}{y(1-x)}-\frac{\left(k_{\perp}+q_{\perp}\right)^{2}}{(1-y)(1-x)}}
$$

Here we have already used $\int d \Lambda^{2} \rho\left(\Lambda^{2}\right)=0, \int d \Lambda^{2} \rho\left(\Lambda^{2}\right)\left(\Lambda^{2}\right)^{D_{\perp} / 2}=0$ to cast $I^{1 \text { Loop }}$ into a rotationally invariant form [22]. Using [note that $p_{1}$ is an energy shell; see Eq. (5.40)]

$$
p_{1}^{2}=-(1-x)\left[\frac{k_{\perp}^{2}}{1-x}+\frac{k_{\perp}^{2}+\lambda^{2}}{x}\right]
$$

one finds

$$
\begin{aligned}
& \operatorname{tr}\left(\Sigma \gamma^{-}\right)=C \int d^{D_{\perp}} k_{\perp} \int d^{D_{\perp}} q_{\perp} \int_{0}^{1} \frac{d x}{x^{2}(1-x)^{2}} \int_{0}^{1} \frac{d y}{y} \int d \Lambda^{2} \rho\left(\Lambda^{2}\right) \\
& \times \frac{k^{2}+\lambda^{2}}{\frac{k^{2}}{1-x}+\frac{k^{2}+\lambda^{2}}{x}} \frac{1}{\frac{k^{2}+\lambda^{2}}{x}+\frac{q^{2}+\Lambda^{2}}{y(1-x)}+\frac{(k+q)^{2}}{(1-y)(1-x)}} \\
& =C \pi^{D_{\perp}} \Gamma\left(1-D_{\perp}\right) \frac{\pi}{\sin \pi\left(D_{\perp} / 2\right)} \int \frac{d \Lambda^{2} \rho\left(\Lambda^{2}\right)}{\left(\Lambda^{2}\right)^{1-D_{\perp}}} \neq 0 \text {. }
\end{aligned}
$$

First and most important, the $\gamma^{+}$component of $\Sigma$ is nonzero and rotational inrariance is thus violated since $p^{-}=0$. Secondly, the result is independent of the outer boson mass $\lambda$; i.e., a Pauli-Villars regularization (with condition $\int d \lambda^{2} \rho\left(\lambda^{2}\right)=0$ ) would have rendered $\operatorname{tr}\left(\Sigma \gamma^{-}\right)$zero.

This is a rather typical result for higher loop graphs and implies the following. Once one has (over-) regularized the short distance singularities so much that one can handle the one-loop singularities in a rotationally invariant way (as in P.-V) then the (milder) higher loop singularities should be no problem any more if one uses the same (over-) regularized versions of the theory there.

It is, however, not sufficient to add only a one-loop counterterm and add wo two-loop counterterms at all, although one might be tempted to do so, because 
e.g., in $2+1$ dimensions the two-loop self-energy of a fermion is finite in a covariant, approach, this violates rotational invariance by a logarithmic divergence. 


\section{REFERENCES for CHAPTER 5}

[1] P. A. M. Dirac, Rev. Mod. Phys. 21, 392 (1949); H. Leutwyler and J. Stern, Ann. Phys. (NY) 112, 94 (1978).

[2] A simple and well-known example is rotational invariance in the context of equal time quantized field theory. The angular momentum operator is a sum of single particle operators and generally approximation schenles preserve rotational invariance.

[3] The situation here is very similar to boost transformations in equal time quantization which mix $\vec{x}$ and $x^{0}$.

[4] However, even in $1+1$ dimensions parity is not manifestly preserved.

[5] Note that we have already introduced one $P-V$ regulator ficld with mass $A$ in order to avoid the ambiguity in the treatment of normal-ordering divergences.

[6] Unfortunately we were not able to prove this in general. It is, however. plausible, since the worst singularities in LC loop calculations occur usually for the $\gamma^{+}$component where they lead to a violation of rotational invariance. We have verified the statement for all relevant two-loop diagrams.

[7] J. D. Bjorken and S. D. Drell, "Relativistic Quantum fields"(McCiraw-Hill, New York, 1966).

[8] H.-C. Pauli and S. J. Brodsky, Phys. Rev. D32, 1993 (1987); 2001 (1987); 'T'. Eller, H.-C. Pauli and S. J. Brodsky, Phys. Rev. D35, 1493 (1987); K. Hornbostel, Ph.D. thesis, SLAC-REPORT--0333 (1989).

[9] It should be emphasized that we could have chosen another basis set for the expansion of the fields. In fact we are always free to replace sums by integrals and thus obtain a continuum formulation. Our choice here does not restrict generality.

(10) A. Tang, Ph.l. thesis, SLAC-PUB-35] (1990). 
[11] In the next section we will construct noncovariant counterterms which display the same structure as the normal-ordering terms. i.e., in the latter approach we can also omit $V_{N O}$ since taking it into account just corresponds to a redefinition of parameters.

[12] S. J. Brodsky, R. Roskies and R. Suaya, Phys. Rev. D8, 4574 (1973).

[13] In this argument refers to the sum of all light-cone timeorderings only.

[14] This is similar to approximations used in equal time approximation which lead to violations of boost invariance.

[15] This is true if all noncovariant terms have been removed in subloops.

[16] In principle further counterterms which have a structure different from terms present in the original Hamiltonian are conceivable.

[17] Note that no wave function renormalization is necessary in the Hamiltonian approach since wave functions are automatically normalized. The normalization constants cancel, due to the Ward identifies, in all physical observables.

[18] C. Bouchiat, P. Fayet and N. Sourlas, Lett. Nuovo Cim. 4, 9 (1972); S.-.J. Chang and T.-M. Yan, Phys. Rev. D7, 1147 (1973).

[19] B. Warr, private communication.

[20] Note that the original instantaneous photon exchange term (3.10) is independent of the photon mass.

[21] Here we have used the Brodsky trick to combine instantaneous fermions with those fermion lines which extend only over one intermediate state by putting the corresponding fermions on energy shell in the numerator.

[22] In fact $I^{1 \text { Loc }}$ can be written as $I^{1 \text { Loop }}=\not p_{1} f\left(p_{1}^{2}\right)$. 


\section{Rotational Invariance in Light-Cone Quantization}

\subsection{Introduction}

Light-cone quantization might be a very valuable tool toward a better understanding of the strong interaction. The main advantages of the formalism are the simple vacuum structure, the manifest boost invariance in the $z$-direction and the Hamiltonian formulation that leads to a very physical approach to field theory.

One of the major disadvantages of the formalism [1] (as for any Hamiltonian form of dynamics) is its nonmanifest Lorentz invariance (here, rotational invariance). Being not manifestly Lorentz covariant one still expects that physical observables $(S$ matrix elements) exhibit the full Lorentz covariance of the underlying Lagrangian. Since the verification of Lorentz covariance of the $S$-matrix in a noncovariant formalism is in general rather tedious, it has become common practice to simply assume covariance of the $S$-matrix in naive light-cone quantization [2]. This paper deals with the problem of Lorentz covariance (in particular, rotational invariance) in light-cone quantization.

A powerful test of rotational invariance is given by examining the angular distribution of the decay products of a heavy scalar particle at rest, such as

$$
\sigma \rightarrow f \bar{f}
$$

Starting out with the light-cone quantized Yukawa modf ( see Chapter 5)

$$
\mathcal{L}=\bar{f}(i \not \partial-m) f+\phi\left(\square+\lambda^{2}\right) \phi+\gamma \bar{f} f \phi
$$

we note that any deviation from a uniform $f \bar{f}$ distribution in physical $S$-matrix elements would indicate a serious violation of rotational invariance. 


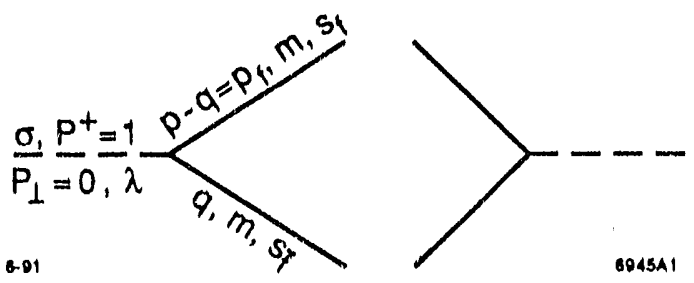

Figure 6.1. Tree level matrix element for the decay $\sigma \rightarrow f \bar{f}$. The dashed line represents a heavy boson with mass $\lambda$ at rest: $p^{+}=p^{-}, p_{\perp}=0$. The sum runs over the fermion (mass $m$ ) spin labels $s_{f}, s_{\bar{f}}$.

This section investigates the decay (6.1) at the one- and two-loop level. A discussion beyond one loop is important in order to decide whether self-induced inertia terms [3], which naturally arise from normal ordering of the Hamiltonian, could cure the problem. Violations at higher loops would mean, in particular, that any clever arrangement of self-induced inertia terms cannot restore a covariant answer for physical S-matrix elements, since self-induced inertias are of second order in the coupling.

We demonstrate an alternative treatment by adding counterterms to the Lagrangian respecting only those symmetries, which are manifestly preserved on the light-cone, i.e., transverse rotations and boosts along the $z$-axis. The goal of this paper is to construct them explicitly and show how rotational invariance can be restored for physical $S$-matrix elements. To complete the discussion, in Section 4 we address the question of why light-cone quantization leads to incorrect results, if naively applied.

\subsection{Breakdown of Covariance at the One-Loop Level and Addition of Noncovariant Counterterms}

We begin our considerations with the decay of a scalar particle into a fermion antifermion pair $\sigma \rightarrow f \bar{f}$ at tree level. The corresponding matrix element squared is (see Fig. 6.1) 


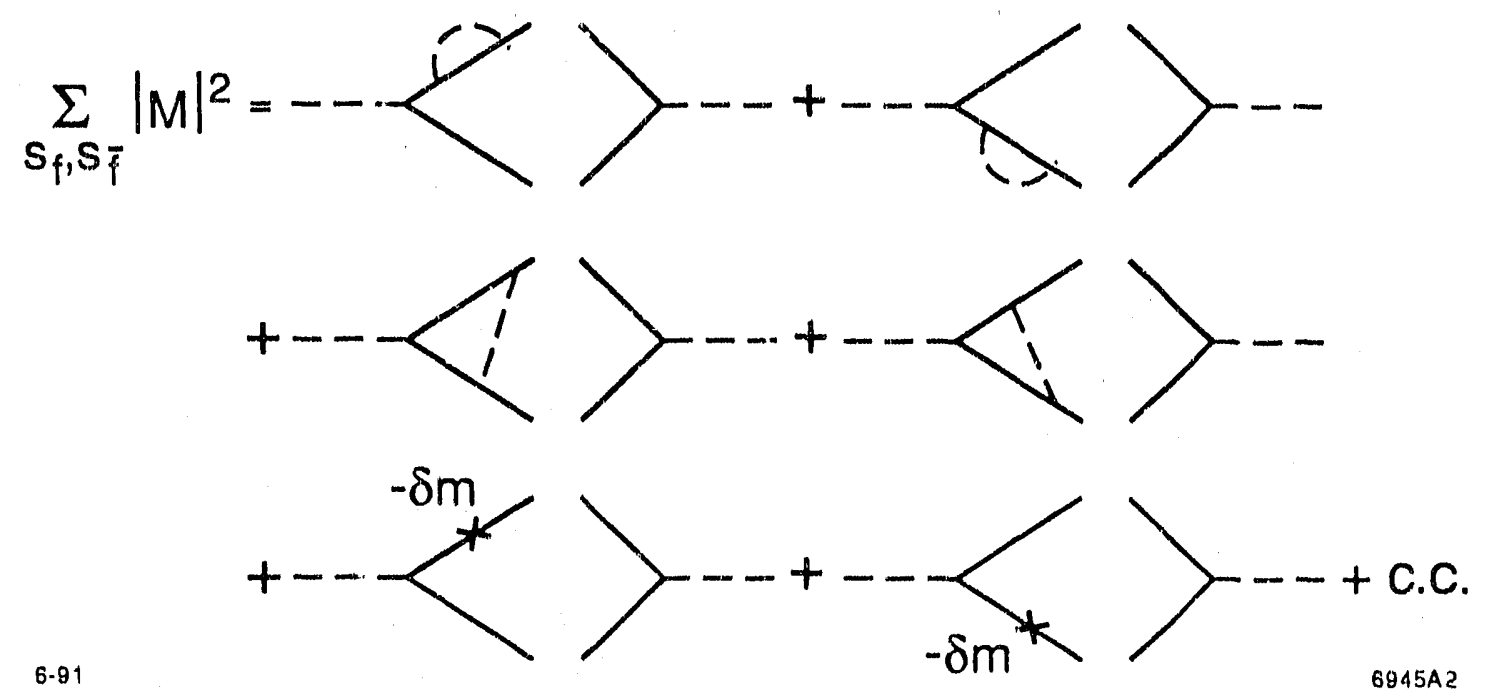

Figure 6.2. Fourth-order contributions to $\sigma \rightarrow f \bar{f}$. The $\delta m$ insertion represents the one-loop mass counterterm.

$$
\sum_{s_{f}, s_{J}}|M|^{2}=\operatorname{Tr}\left(\left(p_{f}+m_{v}\right)\left(-\not h+m_{v}\right)\right) .
$$

Overall light-cone energy conservation constrains the external momenta, leading to

$$
\lambda^{2}=\frac{m^{2}+q_{\perp}^{2}}{q^{+}\left(1-q^{+}\right)} .
$$

Note that, in order to allow for noncovariant counterterms, two different masses have been introduced [4]. A vertex mass $m_{v}$, appears in the numerator, and a kinetic mass $m$, appears in $P^{-}$conservation and in all denominators associated with the diagram [5] (see also previous chapter). Eqs. (6.3) and (6.4) lead immediately to

$$
\sum_{S_{\jmath}, S_{\bar{J}}}|M|^{2}=-2 \frac{m_{v}^{2}-m^{2}}{q^{+}\left(1-q^{+}\right)}-2 \lambda^{2}+8 m_{v}^{2} .
$$

Obviously rotational invariance is obtained if and only if $m_{v}=m$, i.e., no problems arise in tree-level physics.

At the one-loop level the set of diagrams in Fig. 6.2 contributes to the decay. Note that to order $\gamma^{4}$ only interference terms between one-loop and tree level diagrams 


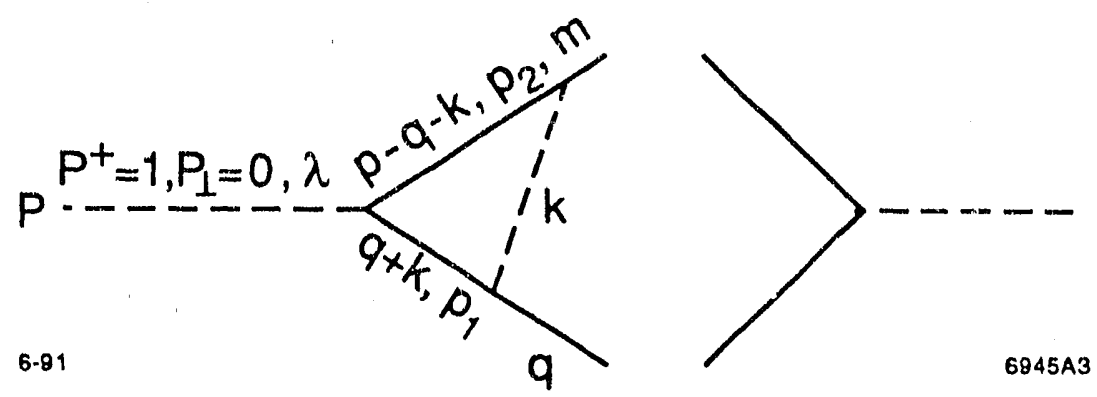

Figure 6.3. Typical contribution to the vertex correction of $\sigma \rightarrow f \bar{f}$.

contribute. As an example we calculate the contribution from interference between a boson-exchange graph aiıd the tree graph (see Fig. 6.3) [6]

$$
\begin{aligned}
& I_{B o s-E x}=\gamma^{4} \int_{0}^{1} \frac{d k^{+}}{\left(16 \pi^{3}\right)} d^{2(1-\epsilon)} k_{\perp} \frac{\theta\left(1-q^{+}-k^{+}\right)}{\left(q^{+}+k^{+}\right)\left(1-q^{+}-k^{+}\right) k^{+}} \\
& \frac{\operatorname{Tr}\left((p-q+m)\left(p_{2}+m\right)\left(-p_{1}+m\right)\left(-q_{1}+m\right)\right)}{\left(p^{-}-\frac{m^{2}+\left(q_{\perp}+k_{\perp}\right)^{2}}{q^{+}+k^{+}}-\frac{m^{2}+\left(q_{\perp}+k_{\perp}\right)^{2}}{1-q^{+}-k^{+}}\right)\left(p^{-}-\frac{m^{2}+\left(q_{\perp}+k_{\perp}\right)^{2}}{1-q^{+}-k^{+}}-\frac{\lambda^{2}+k_{\perp}^{2}}{k^{+}}-\frac{m^{2}+q_{\perp}^{2}}{1-q^{+}}\right)}
\end{aligned}
$$

Using the Brodsky trick [7] to include instantaneous fermion contributions, performing the trace, combining energy denominators and integrating over $k_{\perp}$, we obtain

$$
\begin{gathered}
I_{B o s,-E x .}=\gamma^{4} \int_{0}^{1} \frac{d k^{+}}{16 \pi^{3}} \frac{\theta\left(1-q^{+}-k^{+}\right)}{\left(q^{+}+k^{+}\right)\left(p^{+}-q^{+}-k^{+}\right) k^{+}} \int_{0}^{1} d \alpha \frac{1}{\mu^{2}} \\
\left(\frac{A+B}{2} \frac{\Gamma(\epsilon)}{\left(M^{2}\right)^{\epsilon}}+\frac{C}{M^{2}}\right)
\end{gathered}
$$

where 


$$
\begin{aligned}
\mu= & -\left(\frac{\alpha}{\left(k^{+}+q^{+}\right)\left(1-q^{+}-k^{+}\right)}+\frac{(1-\alpha)\left(1-q^{+}\right)}{k^{+}\left(1-q^{+}-k^{+}\right)}\right) \\
M^{2}= & \mu^{-1} \cdot\left(-q_{\perp}^{2} \mu^{-1}\left(\frac{\alpha}{\left(q^{+}+k^{+}\right)\left(1-q^{+}-k^{+}\right)}+\frac{(1-\alpha)}{\left(1-q^{+}-k^{+}\right)}\right)^{2}\right. \\
& +\alpha\left(p^{-}-\frac{m^{2}+q_{\perp}^{2}}{q^{+}+k^{+}}-\frac{m^{2}+q_{\perp}^{2}}{1-q^{+}-k^{+}}\right) \\
& \left.+(1-\alpha)\left(p^{-}-\frac{m^{2}+q_{\perp}^{2}}{q^{+}}-\frac{\lambda^{2}}{k^{+}}-\frac{m^{2}+q_{\perp}^{2}}{1-k^{+}-q^{+}}\right)\right) \\
A= & \frac{2\left(4 m^{2} q^{+2}-m^{2}-q_{\perp}^{2}\right)}{\left(q^{+}+k^{+}-1\right) q^{+}} \\
B= & \frac{2\left(4 m^{2} q^{+2}-4 m^{2} q^{+}+m^{2}+q_{\perp}^{2}\right)}{\left(1-q^{+}\right) q^{+}} .
\end{aligned}
$$

$C$ acquires terms from zero and linear order in the integration variable $k_{\perp}$ of the Dirac trace. The linear terms give a contribution after shifting momenta. Since the expression is rather lengthy we do not display it here.

Similar steps must be performed for all the other diagrams of Fig. 6.2. This involves renormalizing the diagrams using minimal subtraction and performing the integral over $k^{+}$and $\alpha$ numerically. Then rotational invariance can be checked for the total one-loop $S$-matrix element by computing the diagrams for two different sets of external momenta:

$$
\begin{aligned}
\text { Set (I) }: & q^{+}=\frac{1}{2}, q^{x}=\frac{1}{4}, q^{y}=0 \\
\text { Set (II) }: & q^{+}=\frac{1}{4}, q^{x}=0, q^{y}=0
\end{aligned} .
$$

In both cases, we have chosen $\lambda=1, m=\sqrt{3 / 16}$. Since both sets obey Eq. $(6.3)$ and describe a scalar at rest, i.e., $P^{+}=P^{-}$and $P_{\perp}=0$, the answer is supposed to be the same for both of them, unless rotational invariance is broken. 


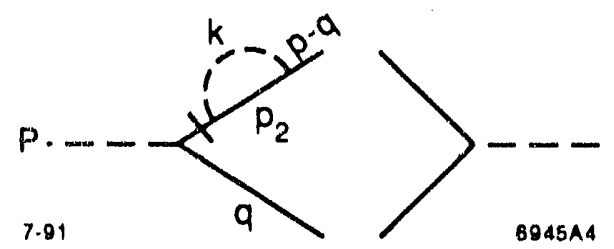

Figure 6.4. Instantaneous contributions to the external self-energy.

For the asymmetry $r$, i.e., the result of the numerical integration for the difference of set (I) and set (II), in in terms of

$$
a=\frac{8 \pi^{3}}{\gamma^{4}} \sum_{s_{\rho} s_{\mathcal{J}}}|M|^{2}
$$

we find $r=0.02 a$. That means rotational invariance is broken for physical $S$-matrix elements at the one-loop level. In Appendix $6 \mathrm{~A}$ we give details of this calculation. In particular it is shown there that the piece which violates rotational invariance comes from the instantaneous contribution in the external self-energy diagrams shown in Fig. 6.4.

In order to keep our discussion as clear as possible, we restrict the number of spacial dimensions to two in what follows. This enables us to disentangle the specific renormalization procedure on the light-cone from the ordinary ones, since the Yukawa model is superrenormalizable in $2+1$ dimensions.

The remaining goal of this section is to show that the term that violates rotational invariance is of the same form as the first term in the r.h.s. of Eq. (6.5). Thus, by allowing independent renormalizations for $m_{v}$ and $m$ one can restore rotational invariance.

Using light-cone perturbation theory (LCPTh) rules one finds [8] for the graph in Fig. 6.4 


$$
\begin{aligned}
I\left(q^{+}, q_{\perp}\right)= & \int_{0}^{1-q^{+}} \frac{d k_{\perp} d k^{+}}{16 \pi^{3}} \\
& \times \frac{\operatorname{Tr}\left((p-\not h+m)\left(p_{2}+m\right) \frac{1}{2} \gamma^{+}\left(-h^{2}+m\right)\right)}{\left(1-q^{+}-k^{+}\right) k^{+}\left(1-q^{+}\right)\left(p^{-}-\frac{m^{2}+q_{\perp}^{2}}{q^{+}}-\frac{\lambda^{2}+k_{\perp}^{2}}{k^{+}}-\frac{m^{2}+\left(q_{\perp}+k_{\perp}\right)^{2}}{1-q^{+}-k^{+}}\right)} .
\end{aligned}
$$

A change of variables $k^{+}=\left(1-q^{+}\right) x, \hat{k}_{\perp}=k_{\perp}+x q_{\perp}$, combined with use of

$$
(p-q)^{2}=p^{-}\left(1-q^{+}\right)-\left(1-q^{+}\right) \frac{m^{2}+q_{\perp}^{2}}{q^{+}}-q_{\perp}^{2}=m^{2}
$$

and

$$
\frac{\lambda^{2}+k_{\perp}^{2}}{x}+\frac{m^{2}+\left(q_{\perp}+k_{\perp}\right)^{2}}{1-x}=\frac{\lambda^{2}}{x}+\frac{m^{2}}{1-x}+\frac{\left(k_{\perp}+q_{\perp} x\right)^{2}+x q_{\perp}^{2}(1-x)}{x(1-x)}
$$

yields

$$
=\int_{0}^{1} \frac{d \widehat{k}_{\perp} d x}{\left(16 \pi^{3}\right)} \frac{\operatorname{Tr}(\ldots)}{x(1-x)\left(m^{2}-\frac{\lambda^{2}}{x}-\frac{m^{2}}{1-x}-\frac{\hat{k}_{\perp}^{2}}{x(1-x)}\right)\left(1-q^{+}\right)}
$$

where $\widehat{k}=k_{\perp}+q_{\perp} x$. To write this in a more compact form, we define the $q^{+}$and $q^{\perp}$ independent function

$$
f(m, \lambda)=\int_{0}^{1} \frac{d \widehat{k}_{\perp} d x}{\left(16 \pi^{3}\right)} \frac{2-x}{\left(x(1-x) m^{2}-\lambda^{2}(1-x)-m^{2} x-\widehat{k}_{\perp}^{2}\right)}
$$

Discarding odd terms in $\widehat{k}_{\perp}$, which do not contribute to the integral, we obtain

$$
\begin{gathered}
I=\int_{0}^{1} d x \frac{d \widehat{k}_{\perp}}{\left(16 \pi^{3}\right)} \frac{(2-x) m\left(1-q^{+}\right)-(2-x) m^{2} q^{+}}{\left(x(1-x) m^{2}-\lambda^{2}(1-x)-m^{2} x-\widehat{k}_{\perp}^{2}\right)\left(1-q^{+}\right)} \\
=\frac{1-2 q^{+}}{1-q^{+}} f(m, \lambda)=\left(2-\frac{1}{1-q^{+}}\right) f(m, \lambda) .
\end{gathered}
$$


A similar calculation for the diagram that correspond to the anti-fermion selfenergy, yields

$$
\widetilde{I}=\left(2-\frac{1}{q^{+}}\right) f(m, \lambda),
$$

which contains the same function $f(m, \lambda)$. The total answer,i.e, the sum of $I$ and $\widetilde{I}$, is

$$
I_{\text {tot }}=\left(4-\frac{1}{q^{+}\left(1-q^{+}\right)}\right) f(m, \lambda)
$$

This result has the remarkable feature that it contains the same $q^{+}$dependence as the term in Eq. (6.5) that violates rotational invariance. Hence the violation of rotational invariance at the one-loop level can be cured by an appropriate renormalization of $m$ and $m_{v}$, i.e., by using different bare values for $m$ and $m_{v}$ in the light-cone Hamiltonian,

\subsection{Breakdown of Covariance at the Two-Loop Level}

In this section it is shown that violations of rotational invariance in the light-cone formulation are not restricted to the one-loop level. This statement is correct even if the one-loop subdivergences are treated covariantly.

In order to constrain the number of diagrams that contribute to the $S$-matrix, we introduce a second fermion flavor and bosons, which change isospin, into the $2+1$ dimensional Yukawa model. However all couplings at fermion-boson vertices are assigned differently, so that isospin symmetry is broken. The new interaction Lagrangian is

$$
\mathcal{L}_{\mathrm{int}}=g_{p n} \bar{p} n \phi_{-}+g_{p p} \bar{p} p \phi_{0}+g_{n n} \bar{n} n \phi_{0}+\text { h.c. }
$$

In this two-flavor model only the rainbow self-energy (Fig. 6.5) and the ladder vertex correction (Fig. 6.6) contribute at order $g_{p p}^{2} \cdot g_{p n}^{4}$ to the decay $\phi_{0} \rightarrow \bar{p} p$. All other 


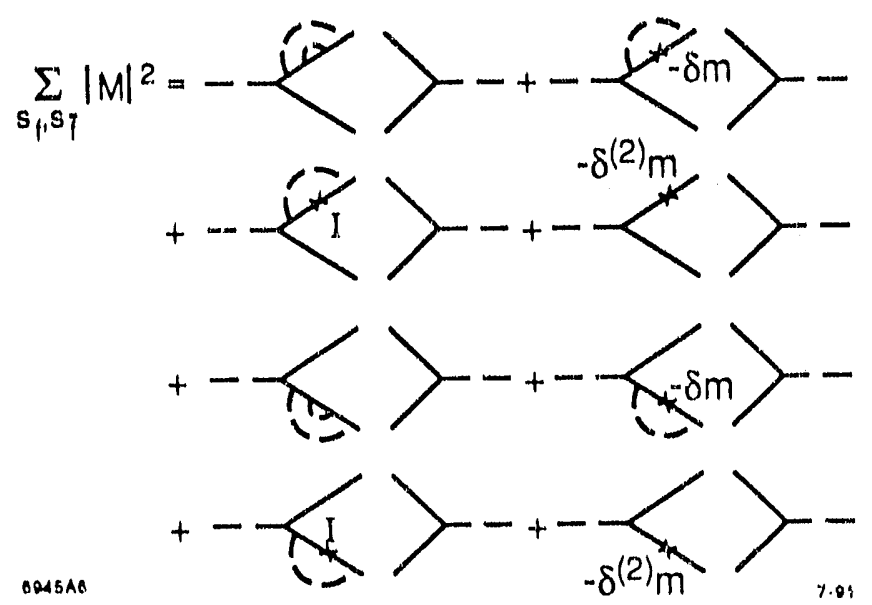

Figure 6.5. Two-loop rainbow self-energy contribution to $\sigma \rightarrow f \bar{f}$. $\delta m, \delta^{(2)} m$ denote the one- and two-loop self-energy mass correction, respectively. I corresponds to a counterterm which restores rotational invariance at the one-loop level.

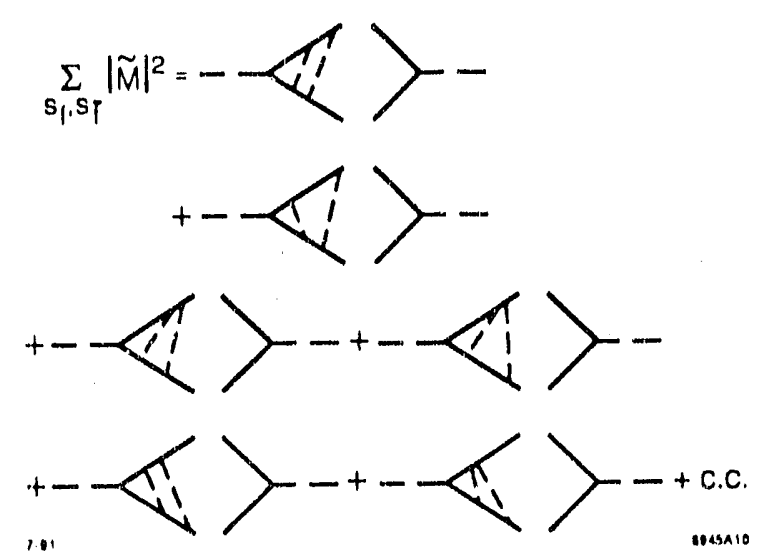

Figure 6.6. Two-loop ladder vertex correction to $\sigma \rightarrow f \bar{f}$. Six timeorderings add up to the covariant answer.

diagrams contribute with other combinations of coupling constants and must be separately covariant, if covariance is assumed for all values of the couplings.

The rainbow self-energy contribution is shown diagramatically in Fig. 6.5. The third diagram restores covariance at the one-loop level. Diagrams which contain $\delta m, \delta^{(2)} m$ are one and two-loop mass counterterms, respectively.

As in Section 2, we consider the instantaneous contribution to the self-energy' diagrams in Fig. 6.7 separately from the rest. Table 6.1 shows the result of the 


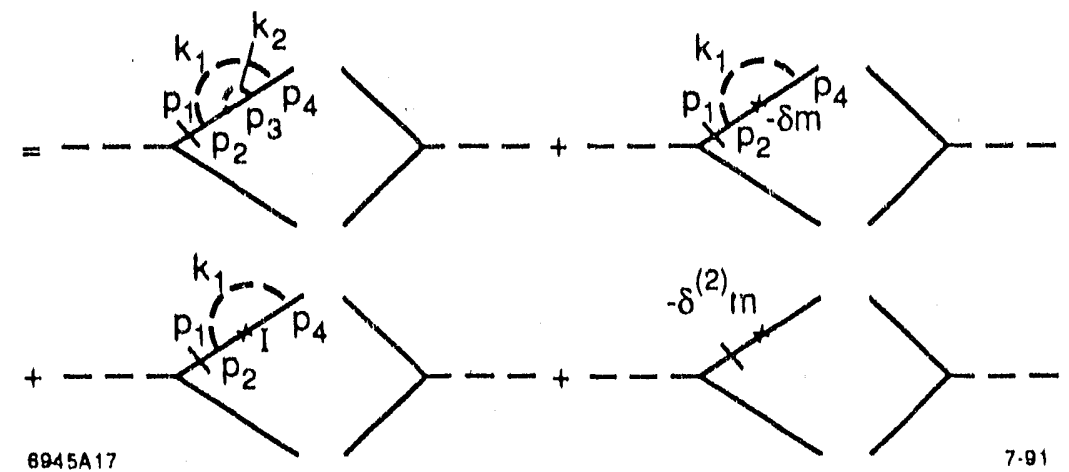

Figure 6.7. Instantaneous self-energy correction in two loops. Momentum labels are assigned as indicated.

Table 6.1. Self-energy contribution to $\sigma \rightarrow f \bar{f}$ in two loops. $a_{2}$ describes the contribution from the instantaneous diagrams (Fig. 6.7), which violate rotational invariance. $a_{1}$ is the result of the numerical integration of the residual self-energy diagrams.

\begin{tabular}{|c|c|c|}
\hline Set & $a_{1}$ & $a_{2}$ \\
\hline (I) & $-1.58 \pm 0.01$ & $0.015 \pm 0.004$ \\
(II) & $-1.58 \pm 0.01$ & $-0.135 \pm 0.002$ \\
\hline
\end{tabular}

Table 6.2. Result of the numerical integration of the ladder vertex correction to $\sigma \rightarrow f \bar{f}$ (Fig. 6.6). A rotational invariant answer is obtained for both sets.

\begin{tabular}{|c|c|}
\hline Set & $a_{1}$ \\
\hline (I) & $-2.13 \pm 0.01$ \\
(II) & $-2.13 \pm 0.01$ \\
\hline
\end{tabular}

numerical integration for both sets of momenta in (2.7). As in the one-loop case, rotational invariance is violated for the instantaneous contribution to the external self-energy diagrams.

The ladder vertex contributions yield the 6 time-orderings shown in Fig. 6.6. The result of the numerical integration is given in Table 6.2 . 


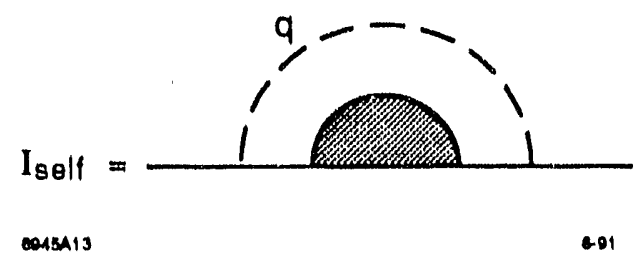

Figure 6.8. $n+1$ loop rainbow self-energy correction.

Thus the ladder diagrams appear to be rotationally invariant by themselves, and a possible cancellation of the noncovariant terms in the self-energy diagram cannot occur. Details of this calculation are given in Appendix 6B [9] .

In the remainder of this section we want to demonstrate that the breakdown of covariance, as in the one-loop case, can be cured by an appropriate renormalization of $m_{v}$ and $m$. Since the calculation is similar to that of the one-loop case, we restrict ourselves to an illustration of this procedure.

We start out with the matrix element in Fig. 6.8 in two loops. In Appendix 6C it is shown that the two-loop self-energy $I_{\text {self }}$ contains a noncovariant piece proportional to $\mathrm{C \gamma}^{+} / \mathrm{p}^{+}$(see also previous chapter), where $C$ is independent of the incoming fermion momentum [10]. Thus, after on-shell mass renormalization, one finds

$$
\begin{aligned}
I_{\text {self }}= & \left(\frac{\gamma^{+}}{p^{+}}-\frac{\bar{u} \gamma^{+} u}{\bar{u} u p^{+}}\right) C \\
& +(p-m) f_{1}\left(p^{2}\right)+\left(p^{2}-m^{2}\right) f_{2}\left(p^{2}\right) .
\end{aligned}
$$

The instantaneous self-energy contribution of Fig. 6.7 becomes

$$
\begin{aligned}
I & =\operatorname{Tr}\left((-k+m) \frac{1}{2} \frac{\gamma^{+}}{p^{+}} \frac{1}{m}(p+m)\right) C \cdot \gamma^{2} \\
& =4 \gamma^{2} C \frac{p^{+}-k^{+}}{p^{+}}=4 \gamma^{2} C \frac{2 p^{+}-1}{p^{+}} \\
& =4 \gamma^{2} C\left(2-\frac{1}{p^{+}}\right)
\end{aligned}
$$


where we have set $p=m$ for the external fermion in Fig. 6.7, and used the following $\gamma^{+} \gamma^{+}=0, k^{+}+p^{+}=1$ and $\bar{u} \gamma^{+} u=2 p^{+}$.

An analogous calculation for the diagram which corresponds to the anti-fermion self-energy, yields

$$
\tilde{I}=4 \gamma^{2} C\left(2-\frac{1}{1-p^{+}}\right)
$$

so that the total contribution becomes

$$
I+\widetilde{I}=4 \gamma^{2} C\left(4-\frac{1}{p^{+}\left(1-p^{+}\right)}\right)
$$

Again we see that Eq. (6.24) has the same form as the piece that violates rotational invariance in Eq. (6.5), which means that rotational invariance can be restored by tuning the vertex mass and the kinetic mass differently [11] .

\subsection{Surface and Zero Mode Contributions}

In the previous sections we have discussed the breakdown of rotational invariance in light-cone quantization and described a way to cure the problem by adding noncovariant counterterms. In order to make the discussion more complete, we will investigate in this section the question of why rotational invariance is broken if lightcone quantization is applied naively. The conclusion will be that naive light-cone quantization omits important surface and zero mode contributions.

We start our discussion with the $n+1$ loop self-energy diagram in Fig. 6.8 in $d$ dimensions and covariant perturbation theory. Since the theory is based on a manifestly covariant Lagrangian, one expects for the $n$-loop self-energy $I_{s e l f}^{n}$ the following structure after mass renormalization

$$
I_{s e l f}^{n}=(\not p-\not q-m) f_{1}^{n}\left((p-q)^{2}\right)+\left[(p-q)^{2}-m^{2}\right] f_{2}^{n}\left((p-q)^{2}\right)
$$


where $f_{i}^{n}$ must have a spectral representation

$$
f_{i}^{n}\left(q^{2}\right)=\int_{s_{0}>0}^{\infty} d s \frac{\rho_{i}^{n}(s)}{q^{2}-s+i \epsilon}
$$

with no poles for $q^{2} \leq 0$. We discuss here only the zero mode effects induced by $f_{1}^{n}$. For $f_{2}^{n}$ the same considerations can be made yielding similar results [12].

One finds for the $f_{1}^{n}$ contribution to the self-energy in $n+1$ loops

$$
I_{\mathrm{self}}^{n+1}=\int \frac{d^{D} q}{(2 \pi)^{D}} \frac{f_{1}^{n}\left((p-\cdots q)^{2}\right)(p-\not q+m)}{\left((p-q)^{2}-m^{2}+i c\right)\left(q^{2}-\lambda^{2}+i c\right)}
$$

Since problems are expected for the $\gamma^{+}$-component only [13], we compute

$$
\begin{aligned}
\frac{1}{D} \operatorname{Tr}\left(\gamma^{-} I_{\text {self }}^{n+1}\right)= & \int \frac{d^{D} q}{(2 \pi)^{D}}\left(p^{-}-\frac{q_{1}^{2}+\lambda^{2}}{q^{+}}\right) \frac{f_{1}^{n}\left((p-q)^{2}\right)}{\left((p-q)^{2}-m^{2}+i \epsilon\right)\left(q^{2}-\lambda^{2}+i \epsilon\right)} \\
& +\int \frac{d^{D} q}{(2 \pi)^{D}} \frac{1}{q^{+}} \frac{f_{1}^{n}\left((p-q)^{2}\right)}{(p-q)^{2}-m^{2}+i \epsilon}
\end{aligned}
$$

where

$$
q^{-}=\frac{1}{q^{+}}\left(q^{2}-\lambda^{2}+\left(q_{\perp}^{2}+\lambda^{2}\right)\right)
$$

was used.

It should be emphasized that even though light-cone variables have been introduced, only algebraical steps have been performed so far, i.e., no breakdown of covariance can have occurred at this point. The trouble occurs when the integration over $q^{-}$is performed, in order to obtain LCPTh.

The first integral in Eq. (6.28) poses problems at the one-loop level, i.e., $f \equiv 1$, when trying to perform the $q^{-}$integration. This is because the integrand falls off no faster than $1 / q^{-}$for $p^{+}-q^{+}=0$ or $q^{+}=0$. Whereas the first case should give rise to a contribution of measure 0 , we expect, nonvanishing contributions from the 
surface term in the second case, since the denominators are multiplied by a function which diverges for $q^{+} \rightarrow 0$.

What we encounter here is nothing else but the one-loop problem of the selfenergy which has been noticed by many authors $[7,14,15]$.

However, in higher loops we expect no trouble arising from this term. 'To illustrate this we use the spectral decomposition of Eq. (6.26) and write the first contribution to Eq. $(6.28)$ as

$$
\begin{aligned}
I_{1}= & \left.\int_{s_{0}}^{\infty} d s \int \frac{d^{D} q}{(2 \pi)^{D}}\left(p^{-}-\frac{q_{1}^{2}+\lambda^{2}}{q^{+}}\right) \frac{\rho_{1}(s)}{\left((p-q)^{2}-s+i \epsilon\right)\left((p-q)^{2}\right.}-m^{2}+i \epsilon\right) \\
& \times \frac{1}{\left(q^{2}-\lambda^{2}+i \epsilon\right)} .
\end{aligned}
$$

If sufficiently regular behavior for $\rho_{1}(s)$ is assumed, the integrand falls off like $\sim$ $\left(1 / q^{-}\right)^{2}$ or faster, which means that surface terms do not contribute [16-18] .

The situation is different for the second integral in Eq. (6.28), however. Performing the $q^{-}$integration leads to [19]

$$
\frac{1}{2} \int d q^{-} d^{D-2} q_{\perp} \frac{1}{q^{+}} \frac{f_{1}^{n}\left((p-q)^{2}\right)}{(p-q)^{2}-m^{2}+i \epsilon}=\frac{1}{p^{+}} \delta\left(p^{+}-q^{+}\right) \int \frac{d^{D} q f_{1}^{n}\left((p-q)^{2}\right)}{(q-p)^{2}+m^{2}+i \epsilon} .
$$

This is because for $p^{+} \neq q^{+}$the contour of the left-hand side can be chosen such that its contribution vanishes. The rest follows from

$$
\frac{1}{2} \int_{0}^{p^{+}} d q^{+} \int d q^{-} d^{D-2} q_{\perp} \frac{1}{q^{+}} \frac{f_{1}^{n}\left((p-q)^{2}\right)}{(p-q)^{2}-m^{2}+i \epsilon}=\frac{1}{p^{+}} \int \frac{d^{D} p f_{1}^{n}\left((p-q)^{2}\right)}{(p-q)^{2}-m^{2}+i \epsilon}
$$

The point is that naive light-cone quantization onits the zero-mode contribution on the right-hand side of Eq. (6.31) and thereby causes a violation of rotational invariance. This also predicts that the piece that violates rotational invariance is 
always proportional to $1 / p^{+}$, which is in perfect agreement with all our experiences at the one, two and three-loop level.

Since the right. hand side of $\mathrm{Eq},(6.31)$ does not depend on the outer boson mass, we see that using a heavy Pauli-..Villars boson regulator instead of dimensional regularization would have taken care of the problem [20].

Io complete this section we want to list again the properties of the diagrams in Fig. 6.8:

- It is very likely that noncovariances appear in any order of perturbation theory.

- The noncovariant piece is always $p_{\perp}$ and $p^{-}$independent and of the form $C\left(\gamma^{+} / p^{+}\right)$.

- The noncovariant zero-mode contribution is independent of tho outer boson mass, which explains why a Pauli-Villars regulator plays an extraordinary role among regulators.

- Dimensional regularization is not sufficient, neither is the so-called "covariant cut-off" [21].

- Even supersymmetric theories suffer from this problem (see Appendix 61)).

\subsection{Summary and conclusions}

We have shown that naive light-cone quantization leads to a violation of rotational invariance in physical $S$-matrix elements. To do this we investigated the decay of a heavy scalar particle at rest and observed a deviation from a uniform distribution of its decay products. The analysis shows that the effect is not restricted to one loop (An explicit example is given in Appendix 6C). Following the general arguments of Section 4 one expects a violation at any order in perturbation theory.

At the one- and two-loop level, we explicitly show that the problem can be cured by tuning the vertex mass $m_{n}$ differently from the kinetic mass $m$. This 
procedure corresponds to adding noncovariant counterterms, which preserve only the kinematic light-cone symmetries. That requires an additional renormalization condition, compared to a manifestly covariant theory.

We suggest the decay of a heavy boson at rest because violation of covariance is obvious in this case. Once the additional counterterm is fixed the statement of renormalizability requires that all processes can be evaluated to the same order in perturbation theory [22] without encountering any further violations [23]. To complete our discussion, we investigated the question of why light-cone quantization goes wrong if it is not applied carefully enough. We found that nonvanishing surface contributions accompanied by a zero mode problem at one loop and missing zero mode contributions at higher loop orders cause a breakdown of the covariant structure of the theory. At this point it should be mentioned that the same problems are expected to occur in gauge theories (in $A^{+}=0$ or any other gauge), quantized on the light-cone. As far as practical methods are concerned, such as DLCQ [24] or the Tamm-Dancoff procedure [3], additional violations of rotational invariance are anticipated. This is because one is forced to work with a finite value of a cut-off which by itself breaks Lorentz invariance. In this paper, we have discussed only those violations of rotational invariance which survive the continuum limit. 


\subsection{Appendix 6A}

Using LCP'Th theory for the self-energy contribution $I_{\text {setf }}$ (Fig. 6.9), one finds

$$
\begin{aligned}
I_{s e l f}= & \frac{\gamma^{4}}{16 \pi^{3}} \int d k^{+} d^{2(1-\epsilon)} k_{\perp} \frac{\Theta\left(1-q^{+}-k^{+}\right)}{\left(1-q^{+}\right) k^{+}\left(1-q^{+}-k^{+}\right)} \\
& \times \frac{\operatorname{Tr}\left(\left(p_{2}+m\right)\left(p_{1}+m\right)\left(p_{2}+m\right)\left(-q_{1}+m\right)\right)}{\left(p^{-}-\frac{m^{2}+q_{\perp}^{2}}{1-q^{+}}-\frac{m^{2}+q_{\perp}^{2}}{q^{+}}\right)\left(p^{--}-\frac{m^{2}+\left(q_{\perp}+k_{1}\right)^{2}}{\left(1-q^{+}-k^{+}\right)}-\frac{k^{2}+\lambda^{2}}{k^{+}}-\frac{m^{2}+q_{\perp}^{2}}{q^{+}}\right)},
\end{aligned}
$$

where $p_{\perp}=0$ and $p^{+}=1$. Note that an off-shell value for $p^{-}$has been assigned in order to deal with the double pole. At the end of the calculation, $p^{-}$is taken on shell. If one shifts variables to

$$
\widetilde{k}_{\perp}=k_{\perp}+q_{\perp} \frac{k^{+}}{1-q^{+}},
$$

the Dirac trace can be reduced to the simple form $A \widetilde{k}_{\perp}^{2}+C$, where

$$
A=2\left(4 m^{2} q^{+}-3 m^{2}+q^{+2} \lambda^{2}-2 q^{+} \lambda^{2}+q_{\perp}^{2}+\lambda^{2}\right) / k_{1}^{+}
$$

and $C$ contains terms of zero and linear order in the integration variable $\widetilde{k_{\perp}}$ of the Dirac trace. This is correct only after terms are discarded which do not contribute to the integral. The linear terms give a contribution after shifting momenta. Since

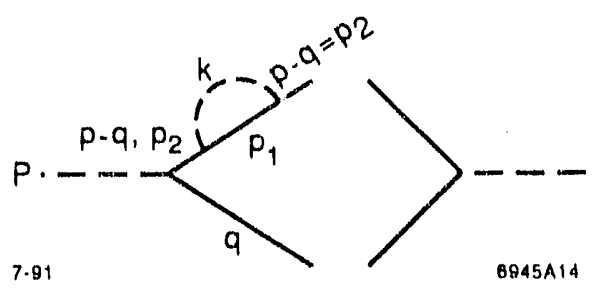

Figure 6.9. Self-energy diagram in one loop. 
the expression is rather lengthy we do not display it here. The $\widetilde{k}_{\perp}$ integration can be trivially performed, yielding

$$
\begin{aligned}
= & -\frac{\gamma^{4}}{16 \pi^{3}} \int_{0}^{1} d k^{+} \frac{\theta\left(1-q^{+}-k^{+}\right)}{\left(1-q^{+}\right)^{2}} \frac{1}{\left(p^{-}-\frac{m^{2}+q_{\perp}^{2}}{1-q^{+}}-\frac{m^{2}+q_{\perp}^{2}}{q^{+}}\right)} \\
& \times\left((1-\epsilon) A \frac{\Gamma(-1+\epsilon)}{\left(M^{2}\right)^{-1+\epsilon}}+C \frac{\Gamma(\epsilon)}{\left(M^{2}\right)^{\epsilon}}\right)
\end{aligned}
$$

where

$$
\begin{aligned}
M^{2}= & -\frac{k^{+}\left(1-k^{+}-q^{+}\right)}{1-q^{+}} \\
& \times\left(q_{\perp}^{2} \frac{k^{+}}{\left(1-q^{+}\right)\left(1-q^{+}-k^{+}\right)}+p^{-}-\frac{\lambda^{2}}{k^{+}}-\frac{m^{2}+q_{\perp}^{2}}{1-q^{+}-k^{+}}-\frac{m^{2}+q_{\perp}^{2}}{q^{+}}\right)
\end{aligned}
$$

$C_{e u l}=0.577 \ldots$ is Euler's constant. The self-energy counterterm that corresponds to the diagrarnm in Fig. 6.10 is evaluated in a similar fashion. As in the self-energy diagram (see Fig. 6.9) the instantaneous contribution is included by putting

$$
p_{2}^{-}=p^{-}-\frac{m^{2}+q_{\perp}^{2}}{q^{+}}
$$

on energy shell. $\delta m$ is given by

$$
\begin{aligned}
\delta m= & \frac{1}{2 m} \gamma^{2} \int d k^{+} d^{(1-\epsilon)} k_{\perp} \frac{1}{k^{+}\left(1-k^{+}\right)} \\
& \times-\frac{\bar{u}\left(p_{1}+m\right) u}{\left(p^{-}-\frac{m^{2}+k_{\perp}^{2}}{1-k^{+}}-\frac{\lambda^{2}+k_{\perp}^{2}}{k^{+}}\right)}
\end{aligned}
$$

with $p_{\perp}=0, p^{+}=1$ for the initial fermion. Note that it does not matter whether the instantaneous contribution is included or not, since it is $k_{\perp}$ independent and therefore gives a vanishing contribution in dimensional regularization. 


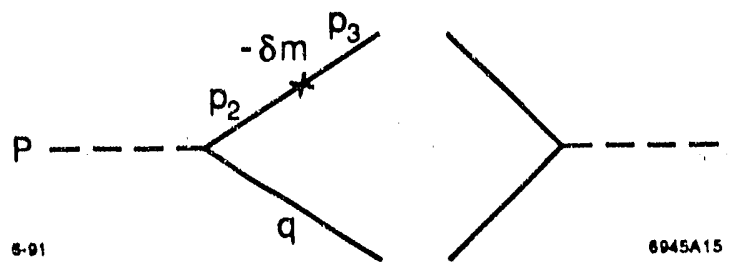

Figure 6.10. One-loop mass correction to the self-energy.

Table 6.3. Total one-loop contribution to $\sigma \rightarrow f \bar{f}$.

\begin{tabular}{|c|c|}
\hline Set & $a_{1}$ \\
\hline (I) & $0.048 \pm 0.2 * 10^{-4}$ \\
(II) & $0.285 \pm 0.6 * 10^{-5}$ \\
\hline
\end{tabular}

Performing steps similar to those taken before one finds

$$
\begin{aligned}
\delta m= & -\frac{\gamma^{2}}{2 m} \int d k^{+}\left(1-\epsilon \ell n N^{2}\right)\left(\frac{1}{\epsilon}-C_{e u l}\right) \\
& \times\left(-\left(\lambda^{2}-m^{2}\right)+m^{2}\left(1-k^{+}\right)+k^{+} m^{2}-2 m^{2}\right)
\end{aligned}
$$

where

$$
N^{2}=-k^{+}\left(1-k^{+}\right)\left(p^{-}-\frac{m^{2}}{1-k^{+}}-\frac{\lambda^{2}}{k^{+}}\right) \text {. }
$$

Table 6.3 shows the result for the numerical integration. The result is that rotational invariance is broken at the one-loop level. Numerically we find that the violating piece arises from the instantaneous self-energy contribution. 


\subsection{Appendix 6B}

We start out with the two-loop rainbow self-energy diagram (Fig. 6.5). LCPTh yields

$$
\begin{aligned}
& I_{\text {rainboul }}=\frac{g_{p p}^{4} g_{p p}^{2}}{\left(16 \pi^{3}\right)^{2}} \int \frac{d k_{1}^{+} d k_{2}^{+} d k_{1 \perp} d k_{2 \perp}}{p_{1}^{+} p_{2}^{+} p_{3}^{+} p_{4}^{+} k_{1}^{+} k_{2}^{+}}\left[\frac{1}{\left(P^{-}-\frac{m^{2}+p_{1}^{2}}{p_{1}^{+}}-\frac{m^{2}+q_{\perp}^{2}}{q^{+}}\right)}\right. \\
& \frac{\operatorname{Tr}\left(\left(p_{f}+m\right)\left(p_{4}+m\right)\left(p_{3}+m\right)\left(p_{2}+m\right)\left(p_{1}+m\right)(-\not h+m)\right)}{D_{1}} \\
& \left.+\frac{\operatorname{Tr}\left(\left(p_{f}+m\right)\left(p_{4}+m\right)(-\delta m)\left(p_{2}+m\right)\left(p_{1}+m\right)(-\not-m+m)\right)}{D_{2}} p_{3}^{+}\right] \\
& -\frac{g_{p p}^{4} g_{p p}^{2}}{\left(16 \pi^{3}\right)^{2}} \int \frac{d k_{1}^{+} d k_{2}^{+} d k_{1 \perp} d k_{2}^{\perp}}{p_{1}^{+} p_{2}^{+} p_{3}^{+} p_{4}^{+} k_{1}^{+} k_{2}^{+}}\left[\frac{1}{\left(P--\frac{m^{2}+p_{1}^{2}}{p_{1}^{+}}-\frac{m^{2}+q_{\perp}^{2}}{q^{+}}\right)}\right. \\
& \left.\operatorname{Tr}\left(\left(p_{f}+m\right)\left(p_{1}+m\right)(-\not h+m)\right) \frac{1}{4 m} \operatorname{Tr}\left(6 p_{4}+m\right)\left(p_{3}+m\right)\left(p_{2}+m\right)\right) \\
& \overline{\left(P_{M, 1}^{-}-k_{1}^{-}-\frac{m^{2}+p_{2 \perp}^{2}}{p_{2}^{+}}\right)\left(P_{M, 1}^{-}-k_{1}^{-}-k_{2}^{-}-\frac{m^{2}+p_{3}^{2}}{p_{3}^{+}}\right)\left(P_{M, 1}^{-}-\frac{m^{2}+p_{3 \perp}^{2}}{p_{1}^{+}}-k_{1}^{-}\right)} \\
& \left.-\frac{\left.\left.\frac{1}{4 m} \operatorname{Tr}\left(\left(p_{f}+m\right)\left(p_{1}+m\right)(-\not++m)\right) \operatorname{Tr}\left(p_{1}+m\right)(-\delta m) \varphi_{2}+m\right)\right)}{\left(P^{-}-\frac{m^{2}+p_{1 \perp}^{2}}{p_{1}^{+}}-\frac{m^{2}+q_{\perp}^{2}}{q^{+}}\right)\left(P_{M, 1}^{-}-k_{1}^{-}-\frac{m^{2}+p_{2 \perp}^{2}}{p_{2}^{+}}\right)\left(P_{M, 1}^{-}-\frac{m^{2}+p_{1 \perp}^{2}}{p_{1}^{+}}-k_{1}^{-}\right)} p_{3}^{+}\right] \\
& +I_{\text {mirrow }}
\end{aligned}
$$

where

$$
\begin{aligned}
& D_{1}=\left(P^{-}-k_{1}^{-}-\frac{m^{2}+p_{2 \perp}^{2}}{p_{2}^{+}}-\frac{m^{2}+q_{\perp}^{2}}{q^{+}}\right) \\
& \left(P^{-}-k_{1}^{-}-k_{2}^{-}-\frac{m^{2}+p_{3 \perp}^{2}}{p_{3}^{+}}-\frac{m^{2}+q_{\perp}^{2}}{q^{+}}\right)\left(P^{-}-\frac{m^{2}+p_{4 \perp}^{2}}{p_{4}^{+}}-\frac{m^{2}+q_{\perp}^{2}}{q^{+}}-k_{1}^{-}\right)
\end{aligned}
$$




$$
\begin{aligned}
D_{2}= & \left(p^{-}-\frac{m^{2}+p_{1 \perp}^{2}}{p_{1}^{+}}-\frac{m^{2}+q_{\perp}^{2}}{q^{+}}\right) \\
& \left(P^{-}-k_{1}^{-}-\frac{m^{2}+p_{2 \perp}^{2}}{p_{2}^{+}}-\frac{m^{2}+q_{\perp}^{2}}{q^{+}}\right)\left(P^{-}-\frac{m^{2}+p_{4 \perp}^{2}}{p_{4}^{+}}-\frac{m^{2}+q_{\perp}^{2}}{q^{+}}-k_{1}^{-}\right)
\end{aligned}
$$

and where $I_{\text {mirrow }}$ denotes the contribution from diagrams in the last two lines of Fig. 6.5. The momenta are given by

$$
\begin{gathered}
p_{1}=\left(1-q^{+}, \lambda^{2}-\frac{m^{2}+q_{\perp}^{2}}{q^{+}},-q_{\perp}\right) \\
p_{2}=p_{4}=\left(1-q^{+}-k_{1}^{+}, \lambda^{2}-\frac{m^{2}+q_{\perp}^{2}}{q^{+}}-k_{1}^{-},-q_{\perp}-k_{1 \perp}\right), \\
p_{3}=\left(1-q^{+}-k_{1}^{+}-k_{2}^{+}, \frac{p_{2}^{-}}{p_{2}^{+}} p_{3}^{+},-q_{\perp}-k_{1 \perp}-k_{2 \perp}\right) \\
\tilde{p}_{2}=\tilde{p}_{4}=\left(1-q^{+}-k_{1}^{+}, \frac{m^{2}+p_{1 \perp}^{2}}{p_{1}^{+}}-k_{1}^{-},-q_{\perp}-k_{1 \perp}\right) \\
\tilde{p}_{3}=\left(1-q^{+}-k_{1}^{+}-k_{2}^{+}, \frac{\tilde{p}_{2}^{-}}{p_{2}^{+}} p_{3}^{+},-q_{\perp}-k_{1 \perp}-k_{2 \perp}\right)
\end{gathered}
$$

and

$$
\begin{gathered}
k_{1}^{-}=\frac{k_{1, \perp}^{2}+\lambda^{2}}{k_{1}^{+}}, \\
k_{2}^{-}=\frac{k_{2, \perp}^{2}+\lambda^{2}}{k_{2}^{+}}, \\
P_{M, 1}^{-}=\frac{p_{1, \perp}^{2}+m^{2}}{p_{1}^{+}}, \\
P^{-}=\lambda^{2} .
\end{gathered}
$$

Note that the third diagram of Fig. 6.5 which restores covariance at the one-loop level can be taken into account by setting $p_{3}^{-}=\left(p_{2}^{-} / p_{2}^{+}\right) p_{3}^{+}$and $\tilde{p}_{3}^{-}=\left(\tilde{p}_{2}^{-} / p_{2}^{+}\right) p_{3}^{+}$. 
This rule relates the bad component of the self-energy $\left(\gamma^{+} p_{1}^{-}\right)$to the good component $\left(\gamma^{-} p_{1}^{+}\right)$and covariance is achieved by construction [25] .

The one-loop mass correction $\delta m$ is given by

$$
\delta m=\frac{e^{2}}{16 \pi^{3}} \int_{0}^{1} d k_{2}^{+} d k_{2 \perp} \frac{\left(1-k_{2}^{+}\right) m^{2}+m^{2}}{\left(-k_{2 \perp}^{2}+k_{2}^{+}\left(1-k_{2}^{+}\right)\left(m^{2}-\frac{m^{2}}{1-k_{2}^{+}}-\frac{\lambda^{2}}{k_{2}^{+}}\right)\right.} .
$$

The last two terms of Eq. (6.42) correspond to the two-loop mass correction $\delta^{(2)} \mathrm{m}$. Note that they are defined quasi-local, i.e., the $\delta^{(2)} m$-subtraction occurs already at. the integrands before integration. This makes the expression suitable for numerical integration.

The instantaneous self-energy contribution can be obtained by subtracting a similar expression like $\mathrm{Eq} .(6.42)$ from $I_{\text {rainbow, }}$, where $p_{1}^{-}$is set on mass shell. The two-loop vertex correction is computed in a similar way. 


\subsection{Appendix 6C}

In this section we show by explicit construction that the two-loop rainbow selfenergy in naive LCPTh contains a noncovariant piece of the form

$$
C \frac{\gamma^{+}}{P^{+}}
$$

even when all subloops have been rendered covariant. $C$ is independent of the incoming fermion momentum $P$. Since by assumption the 1-loop self-energy $I_{\text {self }}^{(1)}$ (Fig. 6.9) is covariant, one should be able to express $I_{\text {self }}^{1}$ in the form $(6.25),(6.26)$. In this particular example we find

$$
\begin{gathered}
\rho_{1}(s)=\frac{e^{2}}{8 \pi^{3}} \Omega(D) \int d x \quad(1-x)(\tau(x))^{\frac{D}{2}-1} \Theta(\tau(x)) \\
\rho_{2}(s)=\frac{e^{2}}{8 \pi^{3}} \Omega(D) \int d x \quad(2-x)(\tau(x))^{\frac{D}{2}-1} \Theta(\tau(x)) \frac{m}{m^{2}-s}
\end{gathered}
$$

where $\tau(x)=\left(x(1-x) s-\left(m^{2} x+\lambda^{2}(1-x)\right)\right.$ was introduced. $\Omega(D)$ is the volume of the D-dimensional unit sphere. Thus in a covariant formalism the 2-loop rainbow self-energy becomes

$$
I^{(2)}=\int_{s_{0}>0}^{\infty} d s \int_{-\infty}^{\infty} \frac{d^{D} k}{(2 \pi)^{D}} \frac{\left(\rho_{1}(s)+(p-k+m) \rho_{2}(s)\right)}{\left(k^{2}-\lambda^{2}+i \epsilon\right)(p-k-m+i \epsilon)\left((p-q)^{2}-s+i \epsilon\right)}
$$

Naive LCPTh replaces $I^{(2)}$ by $I_{l c}^{(2)}$, where

$$
\begin{aligned}
l_{l c}^{(2)}= & \int \frac{d s}{\left(2\left(2 \pi^{2}\right)^{D-1}\right)^{2}} \\
& \frac{\left.\left.\int \frac{d k^{+} d^{D-2} k_{1}}{\left(p^{+}-k^{+}\right)^{2} k^{+}}\left(\rho_{1}(s)+\varphi \rho_{1}+m\right) \rho_{2}(s)\right) \quad \emptyset / 1+m\right)}{\left(p^{-}-\frac{\left(p_{\perp}-k_{\perp}\right)^{2}+m^{2}}{p^{+}-k^{+}}-\frac{k_{\perp}^{2}+\lambda^{2}}{k^{+}}\right)\left(p^{-}-\frac{\left(p_{\perp}-k_{\perp}\right)^{2}+s}{p^{+}-k^{+}}-\frac{k_{\perp}^{2}+\lambda^{2}}{k^{+}}\right)}
\end{aligned}
$$




$$
\begin{aligned}
= & \left.\int \frac{d s}{\left(2(2 \pi)^{D-1}\right)^{2}} \int \frac{d k^{+} d^{D-2} k_{\perp}}{\left(p^{+}-k^{+}\right)^{2} k^{+}}\left(\rho_{1}(s)+\varphi_{1}+m\right) \rho_{2}(s)\right) \\
& \left(p_{1}+m\right) \frac{\left(p^{+}-k^{+}\right)}{s-m^{2}} \\
& \left(\frac{1}{\left(p^{-}-\frac{\left(p_{\perp}-k_{\perp}\right)^{2}+m^{2}}{p^{+}-k^{+}}-\frac{k_{\perp}^{2}+\lambda^{2}}{k^{+}}\right)}-\frac{1}{\left(p^{-}-\frac{\left(p_{\perp}-k_{\perp}\right)^{2}+s}{p^{+}-k^{+}}-\frac{k_{\perp}^{2}+\lambda^{2}}{k^{+}}\right)}\right)
\end{aligned}
$$

and

$$
\hat{p}_{1}=\left(p^{+}-k^{+}, p^{-}-\frac{k_{\perp}^{2}+\lambda^{2}}{k^{+}}-\frac{m^{2}+\left(p_{\perp}-k_{\perp}\right)^{2}}{\left(p^{+}-k^{+}\right)}, p_{\perp}-k_{\perp}\right) .
$$

'The problem is thus reduced to finding the noncovariant piece of the one-loop selfenergy. This has been done [13] and the answer is of the asserted form of Eq. (6.43). 


\subsection{Appendix 6D: The two-loop self-energy in the supersym- metric Wess-Zumino model}

When dimensional regularization is used in the Yukawa model, there is no need for a one-loop noncovariant counterterm if the boson and fermion masses are equal [26]. This observation could be of crucial importance for the light-cone quantization of supersymmetric field theories. In fact, in Ref, 27, it has been proposed to use the (finite [28]) $N=4$ supersymmetric Yang-Mills theory as a regularized extension of light-cone $Q C D_{3+1}$.

Compared to normal theories with similar interactions, supersymmetric theories have a less singular UV-behaviour. Since part of the problem with the violation of rotational invariance is connected with the loop regularization of light-cone singularities, one might hope that SUSY theories are less troubled by noncovariant self-energies. Technically, the improved UV-behaviour arises from cancellations between various diagrams related by SUSY transformations. Perhaps something similar happens with the noncovariant self-energies in light-cone quantization. As mentioned above this is indeed the case at the one-loop level if one uses dimensional regularization in the transverse coordinates. In order to find out whether such a behaviour persists in higher loops, we will investigate the two-loop self-energy of a fermion in the SUSY Wess-Zumino model [29]

$$
\begin{aligned}
\mathcal{L}= & -\frac{1}{2}\left(\partial_{\mu} A\right)^{2}-\frac{1}{2}\left(\partial_{\mu} B\right)^{2}-\frac{1}{2} i \bar{\psi} \gamma^{\mu} \partial_{\mu} \psi \\
& -\frac{1}{2} m^{2} A^{2}-\frac{1}{2} m^{2} B^{2}-\frac{1}{2} m i \bar{\psi} \psi \\
& -g m A\left(A^{2}+B^{2}\right)-\frac{1}{2} g^{2}\left(A^{2}+B^{2}\right)^{2} \\
& -i g \bar{\psi}\left(A-\gamma_{5} B\right) \psi,
\end{aligned}
$$


where $\psi$ is a Majorana spinor and $A$ and $B$ are, respectively, a scalar and a psendoscalar field. The (unsubtracted) one-loop self-energies for bosons and fermions in this model read

$$
\widetilde{\Sigma}_{F}=p_{1} f_{1}\left(p^{2}\right) \quad \widetilde{\Sigma}_{B}=2 p^{2} f_{1}\left(p^{2}\right)
$$

where

$$
f_{1}\left(p^{2}\right)=c \int d^{D-2} k_{\perp} \int_{0}^{1} d x \frac{1-x}{p^{2} x(1-x)-m^{2}-k_{\perp}^{2}+i \epsilon}
$$

( $c$ is some constant). Performing an on-shell mass subtraction one finds [30]

$$
\begin{aligned}
& \Sigma_{F}=(\not p-m) f_{1}\left(p^{2}\right)+\left(p^{2}-m^{2}\right) \frac{f_{2}\left(p^{2}\right)}{m} \\
& \Sigma_{B}=2\left[\left(p^{2}-m^{2}\right) f_{1}\left(p^{2}\right)+\left(p^{2}-m^{2}\right) f_{2}\left(p^{2}\right)\right]
\end{aligned}
$$

where

$$
f_{2}\left(p^{2}\right)=m^{2} \frac{f_{1}\left(p^{2}\right)-f_{1}\left(m^{2}\right)}{p^{2}-m^{2}}
$$

Inserting these one-loop corrections into the one-loop self-energy yields the nested (rainbow-type) contributions to the fermion self energy at $\mathcal{O}\left(g^{4}\right)[31]$

$$
\begin{aligned}
& \Sigma^{1 a}\left(p^{\mu}\right)=\tilde{c} \int d^{D} k \frac{1}{k^{2}-m^{2}+i \epsilon} \frac{(\not p-k) f_{1}\left((p-k)^{2}\right)}{(p-k)^{2}-m^{2}+i \epsilon} \\
& \Sigma^{1 b}\left(p^{\mu}\right)=\tilde{c} \int d^{D} k \frac{1}{k^{2}-m^{2}+i \epsilon} \frac{2(\not p-k) f_{1}\left(k^{2}\right)}{(p-k)^{2}-m^{2}+i \epsilon} \\
& \Sigma^{2 a}\left(p^{\mu}\right)=\hat{c} \int d^{D} k \frac{1}{k^{2}-m^{2}+i \epsilon} \frac{2(\not p-k) f_{2}\left((p-k)^{2}\right)}{(p-k)^{2}-m^{2}+i \epsilon} \\
& \Sigma^{2 b}\left(p^{\mu}\right)=\tilde{c} \int d^{D} k \frac{1}{k^{2}-m^{2}+i \epsilon} \frac{2(\not p-k) f_{2}\left(k^{2}\right)}{(p-k)^{2}-m^{2}+i \epsilon}
\end{aligned}
$$

where $\tilde{c}$ is some constant.

$\Sigma^{a}$ and $\Sigma^{b}$ correspond to insertions of $\Sigma^{1, \text { loop }}$ into the fermion and boson linc, respectively. 
Following Section 4 we substitute in the numerator of the $\gamma^{+}$component

$$
\begin{aligned}
& \Sigma^{1 a}, \Sigma^{2 a}: \quad-k^{-} \mapsto-\frac{k^{2}-m^{2}}{k^{+}}-\frac{k_{\perp}^{2}+m^{2}}{k^{+}} \\
& \Sigma^{1 b}, \Sigma^{2 b}: \quad p^{-}-k^{-} \mapsto \frac{(p-k)^{2}-m^{2}}{\left(p^{+}-k^{+}\right)}+\frac{\left(p_{\perp}-k_{\perp}\right)^{2}+m^{2}}{\left(p^{+}-k^{+}\right)} .
\end{aligned}
$$

As we have shown there naive light-cone quantization (NLCQ) simply neglects the first term thus omitting

$$
\begin{aligned}
& \Delta \Sigma^{1 a}=-\tilde{c} \gamma^{+} \int \frac{d^{D} k}{k^{+}} \frac{f_{1}\left((p-k)^{2}\right)}{(p-k)^{2}-m^{2}}=-\frac{\tilde{c}}{p^{+}} \int d^{D} k \frac{f_{1}\left(k^{2}\right)}{k^{2}-m^{2}} \\
& \Delta \Sigma^{1 b}=2 \frac{\tilde{c}}{p^{+}} \gamma^{+} \int d^{D} k \frac{f_{1}\left(k^{2}\right)}{k^{2}-m^{2}} \\
& \Delta \Sigma^{2 a}=-2 \frac{\tilde{c}}{p^{+}} \gamma^{+} \int d^{D} k \frac{f_{2}\left(k^{2}\right)}{k^{2}-m^{2}} \\
& \Delta \Sigma^{2 b}=2 \frac{\tilde{c}}{p^{+}} \gamma^{+} \int d^{D} k \frac{f_{2}\left(k^{2}\right)}{k^{2}-m^{2}} .
\end{aligned}
$$

One can easily verify that the $\Delta \Sigma$ terms arising from $f_{2}$-insertions cancel whereas this does not happen for $f_{1}$. Thus NLCQ falls short of the correct result by an amount

$$
\Delta \Sigma_{\mathrm{NLCQ}}=\frac{\dot{c}}{p^{+}} \gamma^{+} \int d^{D} k \frac{f_{1}\left(k^{2}\right)}{k^{2}-m^{2}} \neq 0
$$

In the beginning of this appendix we raised the hope that SUSY theories are free of the zero mode problem. Unfortunately this turned out to be false as Eq. (6.54) shows. This means that if one want to use SUSY theories as a regulator for other theories one still has to preregulate them in such a way that there are no noncovariant terms or use some other technique (e.g., noncovariant counterterms) to compensate for $\Delta \Sigma$. This might limit the practical use of SUSY regulators in light-cone quantization considerably. 


\section{REFERENCES for CHAPTER 6}

[1] P. A. M. Dirac, Rev, Mod. Phys. 21, 392 (1949).

[2] For example only certain components of tensors are calculated while other components are constructed on the basis of general Lorentz-covariance arguments.

(3) R. J. Perry, A. Harindranath and K. G. Wilson, Phys. Rev. Lett. 65, 2959 (1990); R. J. Perry and A. Harindranath, Phys. Rev. D43, 492 (1991); R. J. Perry and A. Harindranath, Phys. Rev. D43, 4051 (1991).

(4) In the Hamilton formulation the kinetic mass can be identified with the mass term, that appears in the kinetic energy term for the fermions and the vertex mass is the mass that appears in the helicity-flip fermion boson vertices.

[5] In this simple example there is no energy denominator.

[6] J. Collins, "Renormalization"(Cambridge University Press, Cambridge, 1984), page 66.

[7] S. J. Brodsky, R. Roskies and R. Suaya, Phys. Rev. D8, 4574 (1973).

[8] Invited Lectures, Stellenbosch Advanced Course in Theoretical Physics (Quarks and Leptons), January 1985.

[9] In the case of the ladder diagram, rotational invariance is preserved even for the corresponding off-shell Greens function. The numbers displayed in Table 6.2 have been obtained by setting the fermion mass off-shell and equal to the boson mass in the energy denominators. Numerical difficulties occur in the on shell case at the point $k_{\perp} \simeq 0, k^{+} \simeq 0$ since one energy denominator approaches () in this case. We solved this problem by integrating the difference of set (1) and set (II) directly rather than computing the difference of the integrated values for both sets.

[10] Provided all noncovariant pieces have been removed from subloops. 
[11] It should be noted that this calculation remains correct at higher loops, provided the noncovariant piece has again the structure like $\gamma^{+} / p^{+}$.

[12] In principle, a cancellation between noncovariant terms from $f_{1}^{n}$ and $f_{2}^{n}$ is conceivable. However, in practice we have not encountered an example where this actually happens. In particular, even in SUSY Wess-Zumino model, such terms do not cancel, as the explicit calculation in Appendix 6D demonstrates.

[13] See Chapters 4 and 5.

[14] D. Mustaki, S. Pinsky, J. Shigemitsu and K. Wilson, "Perturbative renormalizaiton of null-plane QED," to appear in Plyss. Rev. D.

[15] A. Thung, Ph.D. thesis, SLAC, REPORT-0351 (1990).

[16] G. Mc Cartor, Z. Phys, C 41, 271 (1988); F. Lenz in "Hadrons and Hadronic Niatter," eds. D. Vautherin, F. Lenz and J. W. Negele, Cargese, France 1989; F. Lenz el al., in Ann. Phys. (NY) 208, 1 (1991), page 1.

[17] It is not clear whether the zero mode terms, which appear in (4.7), are related to those zero modes which are necessary to ensure a proper description of the vacuum structure [16].

[18] Again one has to be careful about $q^{+}=0$ and $q^{+}=p^{+}$. It turns out that the additional term $1 /\left((p-q)^{2}-s+i \epsilon\right)$ ensures sufficiently regular behavior for $q^{+}:=0, q^{-} \rightarrow \infty$ such that no contribution arises from this point.

(19) A similar trick has been used in T.-M. Yan, Phys. Rev. D7, 1780 (1973) as well as in Ref. 8.

[20] (.. Bouchiat, P. Fayet and N. Sourlas, Let.t. al Nuovo Cimeto, 9 (1972); S.-J. Chang and T.-M. Yan, Phys. Rev. D7, 1147 (1973).

[21] The so-called covariant cut-off regularizes a theory by truncating the Hilbert space with respect to a given total invariant mass [15].

[22] Of course by order we mean order in the coupling constant. 
[23] If there are no elementary scalars in the theory one can compare two scatitering experiments in the CM-frame, which are rolated by a nontrivial rotation, like, e.g., the total Compton cross section in QED for insming particles along the z-direction, in comparison to the process where the incoming particles fly along the $x$-direction. In $1+1$ dimensions the breakdown of parily invariance in mave light-cone quantization is conceivable. It would be of interest to check this in a nontrivial example. However, one has to be careful in selecting a process which is really sensitiva to parity violation because quite often C-parity or Isospin symmetry (which are manifest in lightecone cuantization) guarantec parity invariance for certain amplitudes.

[2.1] I.-C. Pauli and S. J. Brodsky, Plys. Rev. D32, 1993 (1987); 2001 (1987); 'T'. Eller, H.-C. Pauli and S. J. Brodsky, Phys. Rev. D35, 1493 (1987); K. Hornbostel, Ph.D. thesis, SLAC-REPORT-0333 (1989); M. Burkardt, Nucl. Phy's. A504, 762(1989).

[25] This method works only if the tensor structure is simple enough, so that different components of the tensor can be related easily. For the vertex correction in QED in Feynman gange this is not the case. A more general subtraction scheme will be presented elsewhere.

[26] This can be directly read off from Eq. (2.9) in Ref. 13.

[27] S. J. Brodsky, invited lectures presented at the 30th Schladming Winter School in Particle Physics; Field Theory, Schladming, Austria, March 1991.

[28] S. Mandelstam, Nucl. Phys. B213, 149 (1983); L. Brink, O. Lindgren and B. E. W. Nilsson, Phys. Lett. 123B, 323 (1983); M. A. Namazic, A. Salam and J. Strathdee, Phys. Rev. D28, 1481 (1983).

[29] J. Wess and B. Zumino, Phys. Lett. 37B, 95 (1971).

[30] It should be emphasized that bosons and fermions acquire the same value for the self-mass corrections. Thus, if we would not perform a mass renormalization 
but rather allow the masses to change via the one-loop correction, this would not induce new noncovariant effects. Snce the masses of bosons and femions would remain equal, the one-loop cancellation of noncovariant ferms still applies.

[31] Of course there are more diagrams contributing to the $\mathcal{O}\left(g^{4}\right)$ self-energy of the "fermion, like e.g., two overlapping loops. However, those kinds of graphs do not give rise to noncovariant self-energies even in non-SUSY theories and can thus be omitted in the discussion here. 


\section{Summary and Outlook}

In the first part of this dissertation a new algorithm for the automatic computation of Feynman diagram amplitudes is presented. The method, which is based on light-cone perturbation theory (LCPTh), is explored and tested for two- and three-loop calculations in QED. The amplitudes are constructed automatically and explicitly, given just the photon connections of the corresponding diagrams. The extension of the algorithm to higher loops is straightforward [1]. In contrast to usual techniques, where single Feynman diagram amplitudes get renormalized, this procedure constructs renormalized amplitudes of sets of Feynman diagrams only. This simplifies the renormalization procedure significantly since those sets can be chosen such that ultraviolet divergences, associated with wavefunction renormalization cancel between diagrams of the same set. Also the infrared behavior is improved in this case, since wavefunction counterierms generally induce artificial infrared divergences. Mass- and coupling constant renormalization must be carried out the usuai way.

In contrast to standard covariant procedures, light-cone field theories involving fermions require further renormalization [5]. In the case of the quadratically divergent one-loop fermion self-energy, this problem has been noticed by many authors $[2,3,4]$. In this dissertation it is shown that additional renormalization is necessary for an infinite number of quadratically divergent LCPTh diagrams at the one-loop level. Also the two-loop order induces additional divergences. This happens even if one-loop subdivergences have been removed consistently from the formalism. It is most likely that additional control of the renormalization procedure is necessary at any order in perturbation theory.

One of the main results of this thesis is that the additional divergences of LCQ can be identified with noncovariant lerms in light-cone quantization. It is shown that those terms lead to observable effects, unless further renormalization is invoked. All 
noncovariant terms that we have encountered in the Yukawa model always had the structure

$$
C \frac{\gamma^{+}}{p^{+}}
$$

where $C$ is independent of the external momenta. In QED we find an additional noncovariant term in the $\perp, \perp$-component of the vacuum polarization. This surprisingly simple structure greatly reduces the number of noncovariant counterterms necessary for the restoration of Lorentz covariance. In the case of QED the burden of fitting nine constants is reduced to just two (one for the $\gamma^{+} / p^{+}$term and the other one for the vacuum polarization).

Another part of this dissertation has been devoted to the analysis of perturbation theory expansions in light-cone field theories. In order to avoid possible subtleties of the quantization procedure for small $x$, we derived the light-cone formulation of Feynman amplitudes by integrating over light-cone energies. The result shows that naive light-cone quantization may omit important surface and zero mode contributions. The analysis recovers the noncovariant term $\gamma^{+} / p^{+}$.

Another objective of this dissertation was to lay down some of the groundwork which is necessary for upcoming nonperturbative studies. The noncovariant counterterms constructed in this work are applicable also for nonperturbative calculations. An essential step is the derivation of renormalization conditions necessary for the adjustment of the additional counterterms. In the case of QED $(2+1)$ (the generalization to QED $(3+1)$ is straightforward) the Hamiltonian, consistent with covariant and gauge invariant perturbation theory was constructed explicitly.

In the following we would like to outline some of the future challenges in nonperturbative light-cone Hamiltonian dynamics. LCPTh provides a useful source of intuition for nonperturbative methods such as DLCQ or the light-front Tamm-Dancoff 
approximation. The connection between nonperturbative questions, i.e., an eigenvalue problem

$$
\left(E-H_{0}\right) \Psi=V \Psi
$$

(the free problem is defined by $H_{0} \Psi_{0}=E_{0} \Psi_{0}$ ) and the language of a perturbation expansion is given by

$$
\Psi=\left(1+\frac{1}{E-H_{0}} V+\frac{1}{E-H_{0}} V \frac{1}{E-H_{0}} V+\cdots\right) \Psi_{0}
$$

since each term in Eq. (7.2) presents a given order in perturbation theory and allows for a depiction in terms of LCPTh diagrams. Nonperturbative Hamiltonian formulations often use an effective version of Eq. (7.1), such as

$$
\left(E-H_{0}\right) \Psi_{f \bar{f}}=V_{e f f} \Psi_{f \bar{f}}
$$

in order to compute physical observables within a certain subspace of Hilbert space (such as $e^{+} e^{-}$or $q \bar{q}$ for fermion-antifermion subspaces in QED and QCD, respectively). The corresponding effective potential $V_{e f f}$ is of infinite order in the coupling constant since Fock states of arbitrarily high particle content can couple to the system under consideration. Most methods, however, such as a finite Tamm-Dancoff truncation, approximate $V_{\text {eff }}$ by a renormalized kernel $\tilde{V}_{\text {eff }}$ which is of finite order in the coupling only. The solution of the corresponding integral equation iterates those kernels ad infinituum and generates LCPTh diagrams up to infinite urder in perturbation theory [see Eq. (7.2)]. The problem is that these diagrams are highly reducible and form a nongauge invariant set thereof. In gauge theories in light-cone gauge this results in $k^{+}$singularities, due to incomplete cancellations of its gauge terms.

A possible solution of this problem might be the addition of nongauge invariant. counterterms to $\tilde{V}_{\text {eff }}$ which could simulate the effect of missing higher-order kernels. 
However, sume of those counterterms are expected to be nonlocal as well as of higher order derivative structure. This could give rise to run-away solutions which would aggravate the numerical treatment of the problem.

With the introduction of $n$ counterterms, $n$ additional parameters $c_{1}, c_{2}, \cdots, c_{n}$ have been introduced into the formalism. If one wants to go beyond the construction of phenomenological models those parameters need to be determined by a set of $n$ constraints such as

$$
<i_{j}\left|f_{k}\left(c_{1}, c_{2}, \cdots, c_{1}\right)\right| i_{l}>=0
$$

where $\left|i_{j}\right\rangle$ corresponds to eigenstates to (7.3) and $k=1, \cdots, n$. The challenge is to construct the functions $f_{k}, k=1, \cdots, n$ explicitly. Possible constraints could be given by current conservation, rotational invariance, and a zero mass for the photon (in case of QED). Note that $\mid i_{j}>$ implicitly depends on $c_{j}, j=1, \cdots, n$ and is of infinite order in the coupling [6]. Thus Eq. (7.4) would determine the new parameters to all orders in the coupling. Another challenge is given by the consistency check for the obtained set of constants $c_{1}, c_{2}, \cdots c_{n}$. A possible way could be the consistent overdetermination by means of further constrains.

The last challlenge to be mentioned in this context concerns the scale-dependence of computed quantities in nonperturbative methods. Scale dependencies are generally introduced through ultraviolet counterterms in the construction of the effective renormalized kernel. At the fixed point of the theory, the parameters can be adjusted (with respect to the scale) such that physical observables are scale independent. In cases where the fixed point of the theory lies in the weak coupling regime, the perturbative $\beta$-function should be recovered.

The central objective of this work was the study of LCPTh as a competitive tool for standard Feynman diagram calculations. The main challenge here is to generalize the algorithm for non-Abelian theories. One might be able to reduce the labor associ- 
ated with the computations of one and two-loop processes in QCD and the Standard Model considerably. Examples of interest are the two-loop corrections to top quark decay, QCD-corrections to charm and beauty production in deep inelastic scattering, as well as the higher loop corrections to the various spin-dependent parton distributions [7]. In particular the last example suggests the use of light-cone quantization, since this is the most natural frame to discribe structure functions. Computing higher-order corrections to these spin-dependent structure functions could also help to clarify whether the Burkardt-Cottingham sum rule [8] is violated.

Finally, we present a list of statements concerning renormalization which have been developed in this dissertation. This list serves as a glossary and provides the reader with the necessary orientation of the results in this work.

- Alternate Denominator (See Appendix A in Chapter 4.) is a method introduced in Ref. [9] which is designed to locally cancel quadratic divergences as well as perform the mass renormalization of fermion self-energies. In order to ensure a consistent description for general fermion self-energy diagram amplitudes, the method must be modified: Contributions where instantaneous fermions are adjacent to the self-energy from the right and left must be excluded from the alternate denominator subtraction. In addition, QED in $A^{+}=0$ gauge requires further restriction to the $g_{\mu \nu}$ piece of the photon propagator only.

- Counterterm. In addition to counterterms which are necessary in a manifestly covariant theory, LCQ requires additional counterterms. This is generally the case even for superrenormalizable theories (see also noncovariant structure of $L C Q$ and Superrenormalizable Models). For the construction of those counterterms see vertex mass, kinetic mass, alternate denominator, nullsubtraction. 
- Dimensional Regularization (See Appendix C in Chapter 4.) can be introduced in LCQ by altering the dimension of perpendicular (with respect to the z-direction) degrees of freedom.

- DLCQ see Theta function regulators in DLCQ.

- Gamma-plus over p- plus terms. (See for example Section 5.4.) In the case of the Yukawa model the noncovariant structure of the theory (see Rotational Invariace) is restricted to terms proportional to $\gamma^{+} / p^{+}$. The same statement applies for Feynman-gauge QED diagrams which contain no vacuum polarization contributions. Noncovariant terms of this kind are due to an improper treatment of surface and zero-mode contributions in LCQ (see Surface and Zero Modes ). They can result in quadratic and logarithmic divergences in LC field theories (see Quadratic Divergence).

- Jellyfish Problem. (See Section 4.2.) Jellyfish diagrams are defined by inserting $n(n \geq 0)$ photons into the one-loop fermion self-energy. In QED) in Feynman gauge jellyfish diagrams give rise to a quadratic divergence see Quadratic Divergence). In the continuum version of $\mathrm{QED}$ in $A^{+}=0$ gauge the problem is reduced to $n=0$ (which is actually just the fermion self-energy). However, the problem can also occur in $A^{+}=0$ gauge since most regularizations of the associated gauge singularity (see light-cone gauge singularity) reduce the small $x$ behavior of the photon propagator to that in Feynman gauge. Independent of that, quadratic divergences are unavoidable in $A^{+}=0$ if questions such as current conservation are investigated. For the removal of the quadratic divergences in the jellyfish graph see Null-Subtraction.

- Light-Cone Gauge Singularity. See Section 4.3.

- Kinetic Mass. See vertex mass.

- Noncovariant Structure of LCQ. See Rotational Invariance. 
- Null Subtraction (See Section 4.2.) is a method which locally subtracts quadratic divergences in n-photon jellyfish diagrams (see jellyfish problem). The subtraction term is given by setting the total light-cone energies and perpmomenta for the bad component of n-photon jellyfish (sub-) diagrams to zero.

- Pauli-Villars Regularization. (See for example Section 5.2.) LCPTh of QED and the Yukawa model is equivalent (at least to two loops) to ordinary covariant Feynman perturbation theory if Pauli--Villars regularization is used. However, unlike regularization in a covariant formalism, where only one PauliVillars photon and fermion are necessary, three ghost particles of each type must be introduced in LCQ. If noncovariant terms (see also Gamma-plus over p-plus) are consistently removed at the one-loop level, higher loop contributions require only one Pauli-Villars particle for each type. Pauli-Villars regularization plays an extraordinary role among regulators in LCQ.

- Quadratic Divergence. (Sce for example Section 4.2.) LCQ involving fermions give rise to two kinds of quadratic divergences (QD)).

1. QD which cancel when all light-cone time-orderings, corresponding to a Feyrman diagram, are summed. Those divergences are due to the lack of absolute convergence in Feynman integrals. A similar problem occurs in ordinary timeordered perturbation theory. A regulator consistent with covariance is essential in this case in order to recover the correct continuum answer. An example is given in the amplitude of diagram 6 in Chapter 4.

2. QD associated with a noncovariant structure of $\mathrm{LCQ}$ (see Rotational Invariance). For the removal of quadratic divergences see Alternate Denomainator, Kinetic Mass, Null-Subtraction, Tensor Method, Vertex Mass, Pauli-Villars Regularization.

- Rotational Invariance. (See for example Chapter 6.) Unless surface--and zero-mode contributions (see surface and zero modes) are treated properly, 
LCQ violates rotational invariance for physical S-matrix elements. Those rotations mix longitudinal with perpendicular degrees of freedom. The problem is not expected to occur for scalar theories with energy-independent interaction vertices (see also Gamma-plus over p-plus terms).

- Self-Induced Inertia (See Section 6.1.) terms arise from normal-ordering of the Hamiltonian and can be interpreted as mass counterterms which naturally arise in $L C Q$. Their treatment is a regulator dependent. In Pauli-Villars regularization fermion self-induced inertias vanish in the case of QED and the Yukawa model. This is due to their independence on the photon (or boson) mass. Self-induced inertias are of second-order in the coupling and do not give rise for a consistent, renormalization in higher loops.

- Superrenormalizable Models. (See Chapter 1.) LCQ of superrenormalizable models such as QED $2+1$, QCD $2+1$ or the Yukawa model in $1+1$ dimensions show divergent structures unfamiliar to manifestly covariant formulations. The cause is given by a breakdown of parity invariance in naive LCQ, which is due to an improper treatment of surface and zero mode contributions (see Surface and Zero Modes).

- Supersymmetry. (See Appendix D in Chapter 6.) The supersymmetric extensions of the Yukawa model, known as the Witten-Zumino-Witten model, does not avoid the necessity of noncovariant counterterms (sec counterterms).

- Surface and Zero Modes (See Section 6.4.) can give rise to nonzero contributions when performing the light-cone energy integration in Feynman integrals. Surface and zero modes are omitted in naive light-cone quantization which can give rise to noncovariant structures of LC field theories (see rotational invariance). This happens when a cancellation of light-cone energies in the numerator and denominators of Feynman diagrams occurs such that all poles lie either in the upper or lower complex-plane. 
- Tensor Method. (See Section 4.2.) In the case of the fermion self-energy in the Yukawa model and QED in Feynman gauge the associated quadratic divergences (see Quadratic Divergence) can be consistently removed by relating the bad component of the self-energy to its good component. Lorentz invariance (see Rotational Invariance) is achieved by construction. The quadratic divergence is avoided in this case. In addition the usual mass renormalization must be carried out. In QED in $A^{+}=0$ the application of the tensor method is rather restricted due to the more complicated Lorentz structure of the selfencrgy. However, in the one-loop self-energy the tensor method can be used when restricted to the $g_{\mu \nu}$ piece of the photon propagator only.

- Theta function regulators in DLCQ. (See Section 4.4.) Theta function regulators limit a function of the total light-cone energy of Fock states. Those regulators generally respect the kinematic symmetries of light-cone quantization but violate rotations which mix longitudinal and transverse degrees of freedom. Theta function regulators do not recover correct continum answers for general perturbative processes, unless noncovariant counterterms are used.

- Vertex Mass. In the Hamiltonian formulation of QED or the Yukawa model the vertex mass of the electron (positron) is defined as the mass appearing in the helicity flip vertex of the theory. In contrast, the kinetic mass appears in the free kinetic energy of the fermion. Whereas the (regularized) bare values for vertex mass and kinetic mass coincide in a manifestly covariant formulation, they must be tuned differently in LCQ in order to offset violations of rotational invariance (see Rotational Invariance and Gamma-plus over p-plus terms). 


\section{REFERENCES for CHAPTER 7}

1. However, the numerical effort necessary to obtain stable and reliable results typically increases by one order of magnitude, at each increasing order in perturbation theory.

2. D. Mustaki, S. Pinsky, J. Shigemitsu and K. Wilson, "Perturbative renormalizaiton of null-plane QED," to appear in Phys. Rev. D.

3. A. Tang, Ph.D. thesis, SLAC-REPORT-0351 (1990).

4. S. J. Brodsky, R. Roskies and R. Suaya, Phys. Rev. D8, 4574 (1973).

5. More generally, problems are expected to occur for theories where light-cone energies appear in numerators of Feynman integrals.

6. In a numerical approach $c_{j}$ might be determined my iteration

7. R. L. Jaffe, Comments Nucl. Part. Phys, 14, 239 (1990).

8. H. Burkardt and W. N. Cottingham, Ann. Phy's. (NY') 56, 453 (1970).

9. S. J. Brodsky, R. Roskies and R. Suaya, Phys. Rev, D8, 4574 (1973). 

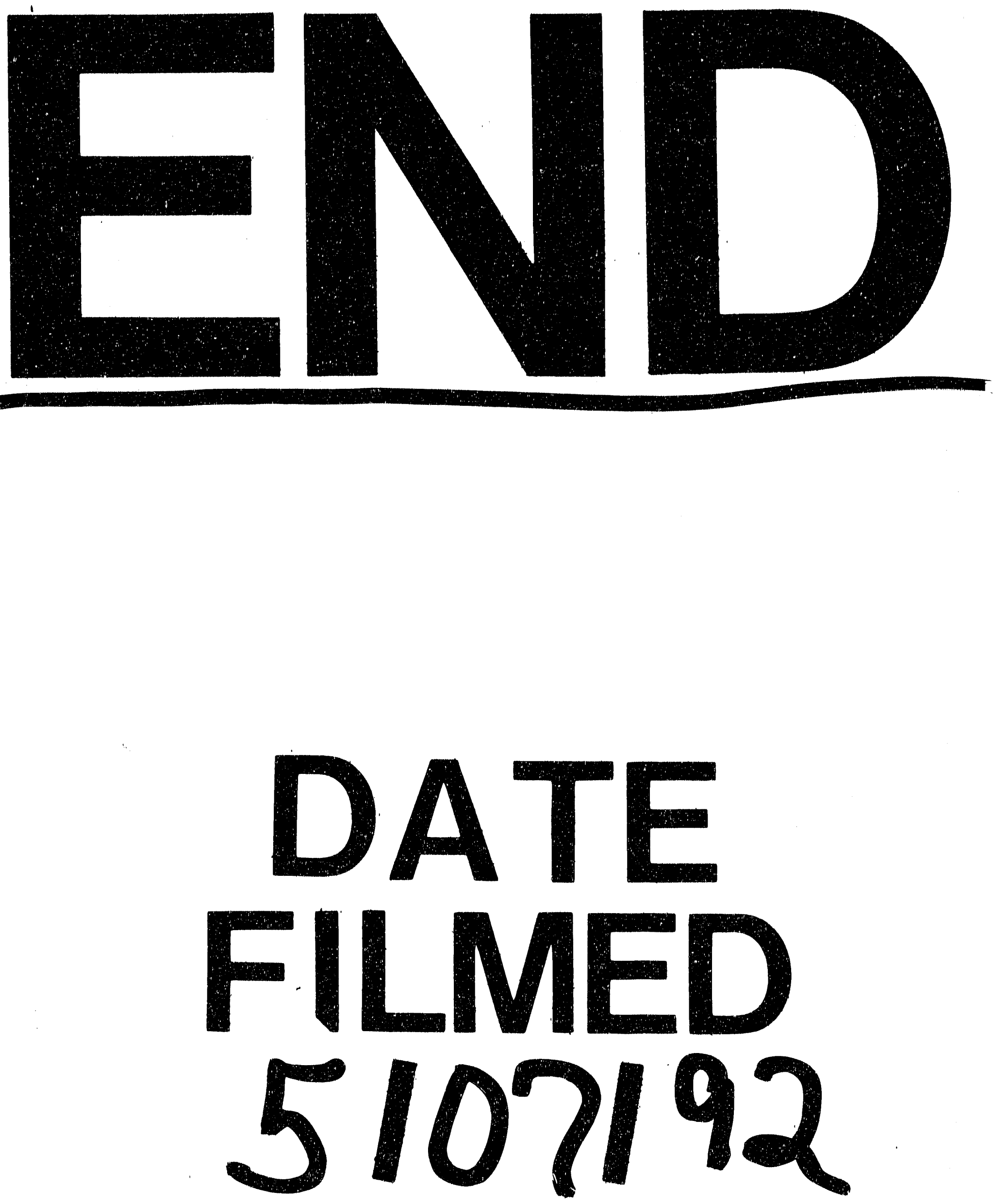
